TRANSACTIONS OF THE

AMERICAN MATHEMATICAL SOCIETY

Volume 361, Number 3, March 2009, Pages 1129-1172

S 0002-9947(08)04539-X

Article electronically published on October 8, 2008

\title{
QUADRATIC DUALS, KOSZUL DUAL FUNCTORS, AND APPLICATIONS
}

\author{
VOLODYMYR MAZORCHUK, SERGE OVSIENKO, AND CATHARINA STROPPEL
}

\begin{abstract}
This paper studies quadratic and Koszul duality for modules over positively graded categories. Typical examples are modules over a path algebra, which is graded by the path length, of a not necessarily finite quiver with relations. We present a very general definition of quadratic and Koszul duality functors backed up by explicit examples. This generalizes the work of Beilinson, Ginzburg, and Soergel, 1996, in two substantial ways: We work in the setup of graded categories, i.e. we allow infinitely many idempotents and also define a "Koszul" duality functor for not necessarily Koszul categories. As an illustration of the techniques we reprove the Koszul duality (Ryom-Hansen, 2004) of translation and Zuckerman functors for the classical category $\mathcal{O}$ in a quite elementary and explicit way. From this we deduce a conjecture of Bernstein, Frenkel, and Khovanov, 1999. As applications we propose a definition of a "Koszul" dual category for integral blocks of Harish-Chandra bimodules and for blocks outside the critical hyperplanes for the Kac-Moody category $\mathcal{O}$.
\end{abstract}

\section{INTRODUCTION}

This paper deals with (categories of) modules over positively graded categories, defines quadratic duality and studies Koszul duality. The first motivation behind this is to get a generalized Koszul or quadratic duality which also works for module categories over not necessarily finite-dimensional, not necessarily unital algebras. In our opinion, the language of modules over (positively) graded categories is very well adapted to this task. Our second motivation is to provide a definition of quadratic duality functors for any quadratic algebra. These functors give rise to the usual Koszul duality functors for Koszul algebras. Remind yourself that a positively graded algebra is Koszul if all simple modules have a linear projective resolution. Despite this definition and the vast amount of literature on Koszul algebras and Koszul duality (see, for example, BGS, GK, GRS, Ke2] and the references therein), and, in particular, its relation to linear resolutions (see, for example, GMRSZ, HI, MVZ and the references therein), it seems that (apart from [MVS] ) there are no attempts to study Koszul duality by working seriously

Received by the editors April 26, 2006.

2000 Mathematics Subject Classification. Primary 16S37, 18E30, 16G20, 17 B67.

The first author was partially supported by the Swedish Research Council.

The second author was partially supported by the Royal Swedish Academy of Sciences and The Swedish Foundation for International Cooperation in Research and Higher Education (STINT).

The third author was supported by The Engineering and Physical Sciences Research Council (EPSRC). 
with the category of linear complexes of projective modules. The intention of the paper is to provide the following:

- A very general definition of a quadratic dual category in terms of the category of linear complexes of projectives, and a detailed study of the latter (Section 3, Section 4).

- A general setup of quadratic/Koszul duality for positively graded categories instead of positively graded algebras (Section 2) with an abstract definition of a duality functor (Section 4) and a Koszul duality theorem for Koszul categories. Using the word duality here might be too optimistic, in particular, since the functors are not even equivalences in general (see Theorem 30). However, later on we will see many "duality-like" effects in our situation, which, from our point of view, justify this usage.

- An illustration of our techniques in the form of an alternative proof of the statement that translation functors and Zuckerman functors for the classical Bernstein-Gelfand-Gelfand category $\mathcal{O}$ are Koszul dual to each other (Theorem 35). This fact is well-known, was conjectured in [BGS] and proved in $[\mathrm{RH}]$ using dg-algebras. Our approach seems to be more elementary and more explicit. As a consequence we prove that twisting/completion and shuffling/coshuffling functors are Koszul dual. Although these functors are well-studied their Koszul duality was a surprise even for specialists. It clarifies the connection of the two categorifications of [BFK] and establishes a direct connection between the main result of [St2] and a result in $\mathrm{Su}$ ] (Section 7).

- An elementary description of the Koszul complex as a complex of C-C!bimodules (see Section A). This provides a connection to the quite recent article [Fl].

- A complimentary approach to Lefèvre's and Keller's generalization of the Koszul duality from [Ke3]. The combination of these two approaches provides a sort of quadratic homological duality.

A (positively) graded category $\mathbf{C}$ is a small category with (positively) graded morphism spaces. To our knowledge, the study of modules over categories was initiated by Bredon $([\mathrm{Br}]$ ) and tom Dieck ( $[\mathrm{tDi}]$ ) in the obstruction theory for finite groups and appears now in different variations; see for example Ga, Mi, Ke2]. In their setup, the categories of (right) modules over a category $\mathbf{C}$ play an important role, where by definition a module is a covariant functor from $\mathbf{C}$ to the category of finite-dimensional vector spaces. From our perspective, modules over a positively graded category $\mathbf{C}$ should be thought of as representations of a (not necessarily unital) positively graded algebra. One could consider $\mathbf{C}$-modules as representations of the (not necessarily finite) quiver with relations associated with $\mathbf{C}$. The vertices of the quiver with relations correspond to the objects in $\mathbf{C}$ and the path algebra $A$ is just the direct sum of all morphism spaces $\bigoplus_{\lambda, \mu} \mathbf{C}(\lambda, \mu)$, where the sum runs over all pairs of objects $\lambda, \mu$ from $\mathbf{C}$. Then, a $\mathbf{C}$-module is a functor which associates to each object in the category (i.e. to each vertex in the quiver) a finite-dimensional vector space and to each morphism (hence to each arrow in the quiver) a linear map between the corresponding vector spaces. The functoriality guarantees that each C-module is exactly what a representation of the associated quiver with relations should be. Note that, in case $\mathbf{C}$ has only finitely many objects, the objects from $\mathbf{C}$ are in bijection with a maximal set of pairwise orthogonal primitive idempotents 
$e_{\lambda}$ of $A$ corresponding to the identity elements in $\mathbf{C}(\lambda, \lambda)$. From the definitions of graded categories (Definition 1) we get a correspondence as follows:

$$
\left\{\begin{array}{l}
\text { positively } \text { graded cat- } \\
\text { egories with finitely } \\
\text { many objects and finite- } \\
\text { dimensional } \\
\text { spaces }
\end{array} \quad \leftrightarrow \quad\left\{\begin{array}{l}
\text { finite-dimensional posi- } \\
\text { tively graded algebras }
\end{array}\right\}\right.
$$

by mapping a category $\mathbf{C}$ to the graded algebra $\bigoplus_{\lambda, \mu \in \mathrm{Ob}(\mathbf{C})} \mathbf{C}(\lambda, \mu)$ of morphisms. In the opposite direction, an algebra $A$ is mapped to the category, whose objects are a chosen system of pairwise orthogonal primitive idempotents and morphisms are the morphisms between the associated indecomposable projective modules.

Under this correspondence equivalent categories correspond to Morita equivalent algebras and isomorphic categories to isomorphic algebras. It is also easy to see that the notions of modules correspond. If we remove the additional finiteness assumptions there is no such nice correspondence, since there is no natural choice for a maximal set of pairwise orthogonal idempotents. Therefore, one should think of graded categories as the correct language to speak about algebras with a fixed set of pairwise orthogonal primitive idempotents (see also [BoGa]). We want to illustrate the results of the paper in the following two examples:

A (well-behaved) illustrating example. We consider the $\mathbb{C}$-algebra $A$ which is the path algebra of the quiver

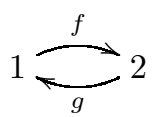

modulo the relation $g \circ f=0$ (i.e. the loop starting at vertex 1 is zero). Putting the arrows in degree one defines a non-negative $\mathbb{Z}$-grading on $A$. We denote this graded algebra by $A$. Note that $A_{0}$ is semi-simple. The algebra $A$ is quadratic and its quadratic dual is the algebra $A !$ given as the path algebra of the same quiver, but with the relation $f \circ g=0$. This algebra is again graded by putting the arrows in degree one. We get decompositions $A=P(1) \oplus P(2)$ and $A^{!}=P^{!}(1) \oplus P^{!}(2)$ into indecomposable (graded) projective $A$-modules corresponding to the vertices of the quiver. Note that the indecomposable projective graded A-modules are all of the form $\mathrm{P}(i)\langle j\rangle$, where $i \in\{1,2\}, j \in \mathbb{Z}$, and $\langle j\rangle$ shifts the grading of the module down by $j$.

Linear complexes of projective A-modules and the equivalence $\epsilon$. To describe the category of finite-dimensional, graded $A$ '-modules we use a result of [MVS] which says that this category is equivalent to $\mathcal{L} \mathcal{C}(\mathrm{P})$, the so-called category of linear complexes of projective modules, i.e. complexes of projective A-modules, where in position $j$ we have a direct sum of projective modules of the form $\mathrm{P}(i)\langle j\rangle$ for $i \in\{1,2\}, j \in \mathbb{Z}$, each occurring with finite multiplicity. The category $\mathcal{L} \mathcal{C}(\mathrm{P})$ is abelian with the usual kernels and cokernels (Proposition 7), and the simple objects are exactly the indecomposable objects $\mathrm{P}(i)\langle j\rangle$, for $i \in\{1,2\}, j \in \mathbb{Z}$, considered as linear complexes with support concentrated in position $j$. Let $\mathrm{S}(i)$ be the simple top of the graded $\mathrm{A}$-module $\mathrm{P}(i)$ and let $\mathrm{I}(i)$ be the injective hull of $\mathrm{S}(i)$. Note that the simple objects in A-gfmod are exactly the $\mathrm{S}(i)\langle j\rangle$, where $i \in\{1,2\}, j \in \mathbb{Z}$. 
Similarly we define $S^{!}(i), \mathrm{P}^{!}(i), \mathrm{I}^{!}(i)$ for the algebra $\mathrm{A}^{!}$. Then the equivalence $\epsilon^{-1}$ : $A !-g M o d \cong \mathcal{L}(P)$ (Theorem 12) gives a correspondence as follows:

\begin{tabular}{|c|c|c|c|c|c|c|c|c|}
\hline$A^{!}-\operatorname{gMod}$ & & & & $\mathrm{C} \mathcal{C}(\mathrm{P}$ & & & & \\
\hline$S^{!}(1)$ & & & 0 & $\rightarrow$ & $\mathrm{P}(1)$ & $\rightarrow$ & 0 & \\
\hline$S^{\prime}(2)$ & & & 0 & $\rightarrow$ & $\mathrm{P}(2)$ & $\rightarrow$ & 0 & \\
\hline$I^{!}(2)$ & 0 & $\rightarrow$ & $\mathrm{P}(1)\langle-1\rangle$ & $\rightarrow$ & $\mathrm{P}(2)$ & $\rightarrow$ & 0 & \\
\hline$! !(1)$ & $\mathrm{P}(1)\langle-2\rangle$ & $\rightarrow$ & $\mathrm{P}(2)\langle-1\rangle$ & $\rightarrow$ & $\mathrm{P}(1)$ & $\rightarrow$ & 0 & \\
\hline$P^{!}(2)$ & & & 0 & $\rightarrow$ & $\mathrm{P}(2)$ & $\rightarrow$ & $P(1)\langle 1\rangle$ & \\
\hline$P^{!}(1)$ & & & 0 & $\rightarrow$ & $\mathrm{P}(1)$ & $\rightarrow$ & $\mathrm{P}(2)\langle 1\rangle$ & $\rightarrow \quad \mathrm{P}(1)\langle 2\rangle$ \\
\hline
\end{tabular}

where the maps in the complexes are the obvious ones and the not shown parts of the complexes are just trivially zero. Note that the indecomposable projective module $\mathrm{P}(i)\langle-j\rangle[j]$ occurs exactly $\left[\mathrm{M}: \mathrm{S}(i)^{!}\langle j\rangle\right]$ times in the complex associated to $M$. The maps in the complexes are naturally obtained from the action of $A !$ on $M$. This equivalence $A^{!}$-gfmod $\cong \mathcal{L} \mathcal{C}(P)$ will be explained in Theorem 12 in the general setup of locally finite-dimensional modules over a quadratic graded category $\mathbf{C}$. In Proposition 11 we will describe the indecomposable injective objects in $\mathcal{L} \mathcal{C}(\mathrm{P})$. It turns out that the injective hull of the simple module $S^{!}(i)$ is nothing other than the maximal linear part of a minimal projective resolution of $\mathrm{S}(i)$. Since the algebra A from our example above is in fact Koszul, the minimal projective resolution of $\mathbf{S}(i)$ is automatically linear. In Proposition 11 we also describe how to get the indecomposable projective objects: We take a minimal injective resolution (for $\mathrm{S}(2)$ we get $\mathrm{I}(2) \rightarrow \mathrm{I}(1)\langle 1\rangle)$, then we apply the inverse of the Nakayama functor (we get $\mathrm{P}(2) \rightarrow \mathrm{P}(1)\langle 1\rangle$ ), and finally we take the maximal linear part of the result (since the resolution in our example is already linear, we are done).

The Koszul self-duality. The algebra A from our example is very special, since it is Koszul self-dual, i.e. A is isomorphic to its quadratic dual A! ([So, Theorem 18] for $\left.\mathfrak{g}=\mathfrak{s l}_{2}\right)$. An isomorphism is of course given by identifying $P(1)$ with $P^{!}(2)$ and $P(2)$ with $P(1)$. In general, the quadratic dual $A$ ! could be very different from A. In Proposition 17 we give a homological characterization of the quadratic dual of a positively graded category. In the example it gets reduced to the fact that $\mathrm{A}^{!} \cong \operatorname{Ext}^{\bullet}(\mathrm{S}(1) \oplus \mathrm{S}(2), \mathrm{S}(1) \oplus \mathrm{S}(2))$, which is the usual Koszul dual. Note that if $A$ is any, not necessarily a finite-dimensional, positively graded algebra (in the sense of Definition (2) of finite global dimension, then its quadratic dual $A$ ! is finite-dimensional (Corollary [19, see also [Ke2, Section 10.4]).

The Koszul dual functors. In Section 5 we define a generalization of (the pair of adjoint) Koszul dual functors (Theorem 222). Using the category of linear complexes of projectives, it is easy to describe the (inverse) Koszul functor $\mathrm{K}_{A}^{\prime}$ (Proposition 21): Given a graded $A !-$-module, the equivalence $\epsilon^{-1}$ maps this module to a linear complex of projective graded A-modules. This can be considered as an object in the bounded derived category $\mathcal{D}^{b}(\mathrm{~A})$ of graded $A$-modules. Hence we have a functor $K_{A(0)}^{\prime}: A^{!}$-gfmod $\rightarrow \mathcal{D}^{b}(A)$ which can easily be extended to a functor defined on $\mathcal{D}^{b}\left(\mathrm{~A}^{!}\right)$. For example, the simple graded $\mathrm{A}^{!}$-modules $\mathrm{S}(i)^{!}$are mapped to the complexes with $\mathrm{P}(i)$ concentrated in degree zero, hence $\mathrm{S}(i) !$ is mapped to $\mathrm{P}(i)$ (see Theorem 22(iii) for a general result). Since the algebra $\mathrm{A}$ in the example is Koszul, so is $A !$, and the Koszul functors are inverse to each other (see [BGS]). This statement will be generalized in Theorem 30 . 
Connection to representation theory of Lie algebras. In Section 6 we consider a special case of [BGS] and [Ba1, namely the Koszul duality functor for blocks of the Bernstein-Gelfand-Gelfand category $\mathcal{O}$ associated to any semi-simple Lie algebra. For the principal block of $\mathcal{O}$ corresponding to the Lie algebra $\mathfrak{s l}_{2}$ we get exactly the category of finitely generated A-modules as described in the examples above. We have the Zuckerman functor $\mathrm{Z}$ which maps an A-module to its maximal quotient containing only simple composition factors of the form $\mathrm{S}(1)$. If $e$ is the primitive idempotent corresponding to $\mathrm{P}(2)$, then $\mathrm{Z}$ is a functor from the category of finitely generated $\mathrm{A}$-modules to the category of finitely generated $\mathrm{A} / \mathrm{A} e \mathrm{~A}$-modules. The functor is right exact and has the obvious right adjoint (exact) functor $i$. If we take the left derived functor of the composition we get $\mathcal{L}(\mathrm{iZ}) \mathrm{S}(2)=\mathrm{S}(1)\langle-1\rangle[1]$ and $\mathcal{L}(\mathrm{iZ}) \mathrm{S}(1) \cong \mathrm{S}(1) \oplus \mathrm{S}(1)\langle-2\rangle[2]$ (see the fourth line of the table above). From the results described above, the Koszul dual functor has to map injective modules to injective modules. It turns out that this is exactly the well-known so-called translation functor through the wall. Section 6 provides an alternative proof of the statement that derived Zuckerman functors and translation functors are Koszul dual. This was conjectured in BGS and proved in the setup of dg-algebras in RH. Our proof avoids the use of dg-algebras, but again illustrates the power of the equivalence $\epsilon$. Theorem 39 finally shows that the left derived functor of $\mathrm{A} e \mathrm{~A} \otimes_{\mathrm{A}} \bullet$, shifted by $\langle 1\rangle$, is Koszul dual to Irving's shuffling functor (【r $]$ ).

Another (less well-behaved) illustrating example. Consider the following quiver:

$$
1 \longleftarrow 2 \longleftarrow 3 \longleftarrow 4 \longleftarrow \cdots .
$$

This defines a positively graded category $\mathbf{C}$ where the objects are the positive integers and the morphisms $\mathbf{C}(m, n)$ are just the linear span of the paths from $m$ to $n$. The length of the path defines a positive grading on $\mathbf{C}$.

The problem with projective covers. The category $\mathbf{C}$-gfmod contains the simple modules $\mathrm{L}(n)\langle k\rangle$ (concentrated in degree $-k, k \in \mathbb{Z}$ ) for any object $n$, and their projective covers $\mathrm{P}(n)\langle k\rangle$ which have $n$ composition factors, namely $\mathrm{L}(j)$ occurs in degree $n-j-k$ for any $1 \leq j \leq n$. The injective hull $\mathrm{I}(j)\langle k\rangle$ of $\mathrm{L}(j)\langle k\rangle$ does not have a finite composition series. The composition factors of $\mathrm{I}(n)\langle k\rangle$ are the $\mathrm{L}(j)$ for $j \geq n$, each appearing once, namely in degree $n-j-k$. Note that this category does not have enough projectives, since, for example, the indecomposable injective modules do not have projective covers. This makes life much more complicated, but it turns out that for any positively graded category we have at least projective covers and injective hulls for any module of finite length, in particular for simple modules (see Lemma 5) and enough projectives in a certain truncated category (Lemma 6). Similar problems can be found e.g. in [AR].

The quadratic dual via linear complexes of projectives. Let us look at the category $\mathcal{L}(\mathrm{P})$, which describes the quadratic dual. The indecomposable injective objects are the linear complexes of the form

$$
\cdots \rightarrow 0 \rightarrow \mathrm{P}(1) \rightarrow 0 \rightarrow \cdots \quad \text { or } \quad \cdots \rightarrow 0 \rightarrow \mathrm{P}(i-1)\langle-1\rangle \rightarrow \mathrm{P}(i) \rightarrow 0 \rightarrow \cdots
$$

for $i \geq 2$ and their $\langle k\rangle[-k]$-shifts for any $k \in \mathbb{Z}$, since they are just the maximal linear parts of the minimal projective resolutions of the simple modules (Proposition (11). The indecomposable projective objects are the linear complexes of the 
form

$$
\cdots \rightarrow 0 \rightarrow \mathrm{P}(i) \rightarrow \mathrm{P}(i+1)\langle 1\rangle \rightarrow 0 \rightarrow \cdots,
$$

for any $i \geq 1$ and together with all their $\langle k\rangle[-k]$-shifts. From the equivalence $\epsilon$ (Theorem [12) we get that the quadratic dual $\mathbf{C}^{!}$is the positively graded category given by the following quiver:

$$
1 \longrightarrow 2 \longrightarrow 3 \longrightarrow 4 \longrightarrow \cdots
$$

with the relation that the composition of two consecutive arrows is always zero. The indecomposable projective module $\mathbf{P}^{!}(i)$ in $\mathbf{C}^{!}$-gfmod therefore has the composition factors $\mathrm{L}^{!}(j)$ for $j=i, i+1$ appearing in degree 0 and 1 , respectively. The indecomposable injective module $\mathbf{I}^{!}(i)$ in $\mathbf{C}^{!}$-gfmod is simple for $i=1$ and has the composition factors $\mathrm{L}^{!}(j)$ for $j=i, i-1$ appearing in degree 0 and -1 , respectively.

The quadratic dual of the quadratic dual. Let us consider the category $\mathcal{L} \mathcal{C}\left(\mathrm{P}^{!}\right)$. The indecomposable injective objects are the linear complexes of the form

$$
\cdots \rightarrow \mathrm{P}(i+2)\langle-2\rangle \rightarrow \mathrm{P}(i+1)\langle-1\rangle \rightarrow \mathrm{P}(i) \rightarrow 0 \rightarrow \cdots
$$

for $i \geq 1$, and their $\langle k\rangle[-k]$-shifts for any $k \in \mathbb{Z}$. Since the projective resolutions of the simple modules are linear, these are nothing other than the projective resolutions of the simple $\mathbf{C}$ !-modules, and the category $\mathbf{C}$ ! is Koszul. The indecomposable injective objects are the linear complexes of the form

$$
\cdots \rightarrow 0 \rightarrow \mathrm{P}(i) \rightarrow \mathrm{P}(i-1)\langle 1\rangle \rightarrow \cdots \rightarrow \mathrm{P}(1)\langle i-1\rangle \rightarrow 0 \rightarrow \cdots,
$$

for any $i \geq 1$ together with their $\langle k\rangle[-k]$ shifts. From Theorem 12 we get that the quadratic dual $\mathbf{C}^{!}$is the positively graded category given by the quiver (1.3). Note that $\mathbf{C}$ and its quadratic dual are both Koszul. The (inverse) Koszul duality functor $K_{\mathbf{C}}^{\prime}$ is again nothing other than extending $\epsilon$ to a functor defined on the corresponding derived category mapping a complex of locally finite-dimensional graded $\mathbf{C}^{!}$-modules to a complex of linear complexes of projectives. Taking the total complex we get a complex of locally finite-dimensional graded $\mathbf{C}$-modules. This description of the (inverse) Koszul duality functor can be found in Proposition 21.

A (classical) family with the same quadratic duals. Consider the algebra $B(\infty)=\mathbb{C}[x]$ or $B(n)=\mathbb{C}[x] /\left(x^{n}\right)$ for any integer $n \geq 3$. Putting $x$ in degree 1 we get a graded algebra $\mathrm{B}(n)$ for $n \geq 3$ or $n=\infty$. The maximal linear part of a minimal projective resolution of the trivial $\mathrm{B}(n)$-module is just the complex $\mathrm{B}(n)\langle-1\rangle \stackrel{x}{\rightarrow} \mathrm{B}(n)$. By Proposition 11 and Theorem 12 we get a description of the (only) indecomposable injective $\mathrm{B}(n)$-module. In particular, $\mathrm{B}(n)^{!} \cong \mathbb{C}[x] /\left(x^{2}\right)$, independent of $n$. Proposition 11 and Theorem 12 also imply $\left(\mathrm{B}(n)^{!}\right)^{!} \cong\left(\mathbb{C}[x] /\left(x^{2}\right)\right)^{!} \cong \mathbb{C}[x]$ which is the classical example of Koszul duality from BGG1 for $n=2$.

A (too badly behaved) illustrating example. Consider the path algebra $A$ of the following quiver:

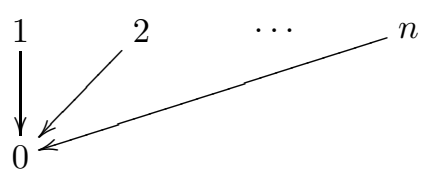


(i.e. vertices are $\{0,1,2, \ldots\}$ and for each $i>0$ there is an arrow $i \rightarrow 0$ ). Putting the arrows in degree one defines a non-negative grading $\mathrm{A}$ on $A$. However, this example is different from the previous ones because there are infinitely many arrows pointing to the vertex 0 . Hence the morphism space from $\mathrm{P}(0)$ to $\bigoplus_{i \geq 0} \mathrm{P}(i)$ is infinite-dimensional in degree one. This infinite-dimensionality makes some of our arguments inapplicable. Hence we will avoid such situations in our paper by considering locally bounded categories (condition (C-iv) in Subsection 2.1; cf. e.g. [BoGa, 2.1]).

User's manual. The following section contains basic definitions and results on graded categories which are crucial for the general approach but are quite technical. Therefore, at the first reading attempt, we suggest skipping all the details from Section 2 and carrying on with Section 3 Since our paper is rather long and contains lots of notation for objects of rather different nature, we tried, for the reader's convenience, to organize our notation in a way as unified as possible via different fonts. Of course there are exceptions due to already well-established notation in the literature, but otherwise the general convention for notation in the paper is as follows:

\begin{tabular}{|l|l|}
\hline Object & Notation \\
\hline \hline Algebras: & $A, B, C, \ldots$ \\
\hline Graded algebras: & $\mathrm{A}, \mathrm{B}, \mathrm{C}, \ldots$ \\
\hline Categories: & $\mathcal{A}, \mathcal{B}, \mathcal{C}, \ldots$ \\
\hline Graded categories: & $\mathbf{A}, \mathbf{B}, \mathbf{C}, \ldots$ \\
\hline Modules: & $M, N, L, \ldots$ \\
\hline Graded modules: & $\mathrm{M}, \mathrm{N}, \mathrm{L}, \ldots$ \\
\hline Complexes: & $\mathcal{X}^{\bullet}, \mathcal{Y}^{\bullet}, \mathcal{Z}^{\bullet}, \ldots$ \\
\hline Functors: & $\mathrm{F}, \mathrm{G}, \mathrm{K}, \ldots$ \\
\hline Derived categories and functors: & $\mathcal{D}, \mathcal{L}, \mathcal{R}, \ldots$ \\
\hline Dualities: & $\mathbb{D}, \mathbf{d}, \ldots$ \\
\hline Objects in categories: & $\lambda, \mu, \nu, \ldots$ \\
\hline Idempotents: & $e, e_{\lambda}, \ldots$ \\
\hline
\end{tabular}

\section{Preliminaries}

For the whole paper we fix an arbitrary field $\mathbb{k}$. Throughout the paper graded means $\mathbb{Z}$-graded, and algebra means, if not otherwise stated, a unital $\mathbb{k}$-algebra with unit 1 ; dim means $\operatorname{dim}_{\mathbb{k}}$, and a category means a small category. For any category $\mathcal{A}$ we denote by $\operatorname{Ob}(\mathcal{A})$ the set of objects of $\mathcal{A}$ and often just write $\lambda \in \mathcal{A}$ if $\lambda \in \operatorname{Ob}(\mathcal{A})$. For $\lambda, \mu \in \mathcal{A}$ the morphisms from $\lambda$ to $\mu$ are denoted $\mathcal{A}(\lambda, \mu)$. We denote by $\mathcal{A}^{\text {op }}$ the opposite category, that is, $\mathcal{A}^{o p}(\lambda, \mu)=\mathcal{A}(\mu, \lambda)$. If not stated otherwise, functors are always covariant.

2.1. Graded algebras and graded categories. Let $\mathbf{C}$ be a $\mathbb{k}$-linear category with a set of objects $\mathrm{Ob}(\mathbf{C})$. Let $e_{\lambda} \in \mathbf{C}(\lambda, \lambda)$ be the identity morphism. Recall that the category $\mathbf{C}$ is called graded provided that the morphism spaces are graded, that is, $\mathbf{C}(\lambda, \mu)=\bigoplus_{i \in \mathbb{Z}} \mathbf{C}_{i}(\lambda, \mu)$ such that $\mathbf{C}_{i}(\mu, \nu) \mathbf{C}_{j}(\lambda, \mu) \subseteq \mathbf{C}_{i+j}(\lambda, \nu)$ for all $\lambda, \mu, \nu \in \mathrm{Ob}(\mathbf{C})$ and $i, j \in \mathbb{Z}$.

A standard example: A standard example of a graded category is the category with objects finite-dimensional graded $\mathbb{k}$-vector spaces and morphisms the $\mathbb{k}$-linear 
maps. The $i$-th graded part is then given by graded maps which are homogeneous of degree $i$.

$A$ rather naive example: To any graded $\mathbb{k}$-algebra $\mathrm{A}=\bigoplus_{i \in \mathbb{Z}} \mathrm{A}_{i}$ one can associate, in a rather naive way, the graded category $C^{A}$ containing one single object, namely A. The morphisms in this category are given by putting $\mathbf{C}_{i}^{\mathrm{A}}(\mathbf{A}, \mathrm{A})=\mathrm{A}_{i}$ for all $i \in \mathbb{Z}$ with compositions given by the multiplication in $A$. This example will not be very important for us, although it appears quite often in the literature; namely whenever a discrete group is considered as a category (groupoid) with one object and morphisms given by the elements of the group and composition given by the group multiplication. If $\mathbf{C}$ is a graded $\mathbb{k}$-linear category, one can consider $\bigoplus_{\lambda, \mu \in \mathrm{Ob}(\mathbf{C})} \mathbf{C}(\lambda, \mu)$, which is a graded $\mathbb{k}$-algebra, however without a unit element if $|\mathrm{Ob}(\mathbf{C})|=\infty$. As already mentioned in the Introduction, this procedure does not have a uniquely defined inverse in general.

From graded categories to quotient categories and vice versa. Graded categories appear as quotient categories modulo free $\mathbb{Z}$-actions. There is even a correspondence

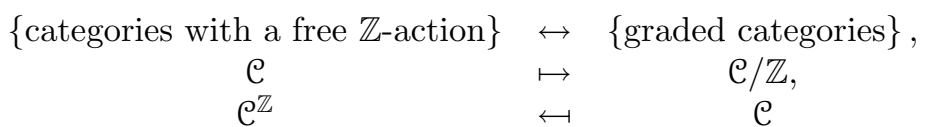

constructed in the following way: Let $\mathcal{C}$ be a $\mathbb{k}$-category. Assume that the group $\mathbb{Z}$ acts freely on $\mathcal{C}$ via automorphisms (here freely means that the stabilizer of every object is trivial). In this case we can define the quotient category $\mathrm{C} / \mathbb{Z}$, whose objects are the orbits of $\mathbb{Z}$ on $\mathrm{Ob}(\mathcal{C})$, and for $\lambda, \mu \in \mathrm{Ob}(\mathcal{C})$ the morphism set $\mathcal{C} / \mathbb{Z}(\mathbb{Z} \lambda, \mathbb{Z} \mu)$ is defined as the quotient of

$$
\bigoplus_{\substack{\lambda^{\prime} \in \mathbb{Z} \lambda \\ \mu^{\prime} \in \mathbb{Z} \mu}} \mathcal{C}\left(\lambda^{\prime}, \mu^{\prime}\right)
$$

modulo the subspace, generated by all expressions $f-i \cdot f$, where $i \in \mathbb{Z}$. The product of morphisms is defined in the obvious way. For any $\lambda, \mu \in \mathrm{Ob}(\mathcal{C})$ we have a canonical isomorphism of vector spaces,

$$
\mathcal{C} / \mathbb{Z}(\mathbb{Z} \lambda, \mathbb{Z} \mu) \cong \bigoplus_{\lambda^{\prime} \in \mathbb{Z} \lambda} \mathcal{C}\left(\lambda^{\prime}, \mu\right),
$$

which turns $\mathcal{C} / \mathbb{Z}$ into a graded category. Conversely, let $\mathcal{C}$ be a graded $\mathbb{k}$-category. Then we can consider the category $\mathcal{C}^{\mathbb{Z}}$ such that $\mathrm{Ob}\left(\mathcal{C}^{\mathbb{Z}}\right)=\mathrm{Ob}(\mathcal{C}) \times \mathbb{Z}$, and for $\lambda, \mu \in \mathrm{Ob}(\mathcal{C})$ and $i, j \in \mathbb{Z}$ we have $\mathcal{C}^{\mathbb{Z}}((\lambda, i),(\mu, j))=\mathcal{C}_{j-i}(\lambda, \mu)$. Then $\mathbb{Z}$ acts freely on $\mathcal{C}^{\mathbb{Z}}$ in the obvious way and we have $\mathcal{C}^{\mathbb{Z}} / \mathbb{Z} \cong \mathcal{C}$ as graded categories (for details we refer the reader, for instance, to [CM, Section 2]).

Positively graded algebras and categories. In the following it will be useful to strengthen the definition of a graded category and replace it with the notion of a positively graded category defined as follows (compare with the notion of locally bounded categories in [BG, 2.1]):

Definition 1. A graded $\mathbb{k}$-category $\mathbf{C}$ is said to be positively graded provided that the following conditions are satisfied:

(C-i) $\mathbf{C}_{i}(\lambda, \mu)=0$ for all $\lambda, \mu \in \mathrm{Ob}(\mathbf{C})$ and $i<0$.

(C-ii) $\mathbf{C}_{0}(\lambda, \mu)= \begin{cases}0, & \text { if } \lambda \neq \mu, \\ \mathbb{k} e_{\lambda}, & \text { if } \lambda=\mu .\end{cases}$ 
(C-iii) $\operatorname{dim} \mathbf{C}_{i}(\lambda, \mu)<\infty$ for all $\lambda, \mu \in \mathrm{Ob}(\mathbf{C})$ and $i \in \mathbb{Z}$.

(C-iv) For any $\lambda \in \operatorname{Ob}(\mathbf{C}), i \in \mathbb{Z}$, the sets $\left\{\mu \mid \mathbf{C}_{i}(\lambda, \mu) \neq\{0\}\right\}$ and $\left\{\mu \mid \mathbf{C}_{i}(\mu, \lambda) \neq\right.$ $\{0\}\}$ are finite.

A semi-simple category is always positively graded, whereas the category of all finite-dimensional graded $\mathbb{k}$-vector spaces is not positively graded (both (C-ii) and (C-iii) fail). For other examples of positively graded categories we refer to the Introduction, where one also finds an example of a category, which does not satisfy the condition (C-iv). We remark that a positively graded category is in reality nonnegatively graded (since (C-i) only says that all negatively graded components are zero); however, the use of the term positively graded in this context is now commonly accepted (see for example [BGS, 2.3] or [MVS, Introduction]). Positively graded categories with finitely many objects come along with positively graded algebras:

Definition 2. A graded algebra, $A=\bigoplus_{i \in \mathbb{Z}} A_{i}$, is said to be positively graded provided that the following conditions are satisfied:

(A-i) $\operatorname{dim} \mathrm{A}_{i}<\infty$ for all $i \in \mathbb{Z}$.

(A-ii) $\mathrm{A}_{i}=0$ for all $i<0$.

(A-iii) $\mathrm{A}_{0}=\bigoplus_{\lambda \in \Lambda} \mathbb{k} e_{\lambda}$, where $1=\sum_{\lambda \in \Lambda} e_{\lambda}$ is a (fixed) decomposition of the unit element 1 into a finite sum of pairwise orthogonal primitive idempotents.

As already mentioned in the Introduction, to any positively graded algebra A one associates a positively graded $\mathbb{k}$-category, which we denote by $\mathbf{A}$. The objects of this category are $\operatorname{Ob}(\mathbf{A})=\Lambda$ (one can also interpret these objects as indecomposable projective right $\mathbf{A}$-modules), and the morphisms are defined by setting $\mathbf{A}_{i}(\mu, \lambda)=$ $e_{\lambda} \mathrm{A}_{i} e_{\mu}$ for all $\lambda, \mu \in \mathrm{Ob}(\mathbf{A})$ and $i \in \mathbb{Z}$ (in other words, the morphisms are just the homomorphisms between the corresponding projective modules). The composition of morphisms in $\mathbf{A}$ is induced by the multiplication in $\mathbf{A}$. The condition (C-iv) is satisfied, since we have finitely many objects. Conversely, for any positively graded $\mathbb{k}$-linear category $\mathbf{C}$ with finitely many objects the space $\bigoplus_{\lambda, \mu \in \mathrm{Ob}(\mathbf{C})} \mathbf{C}(\lambda, \mu)$ is a positively graded $\mathbb{k}$-algebra. These two processes restrict naturally to the correspondence described in (1.1) which will always be in the background of our considerations. However, the setup of graded categories is more general, since we also allow $|\mathrm{Ob}(\mathbf{C})|=\infty$.

2.2. Modules over graded categories. We have seen that positively graded categories correspond to positively graded algebras in the sense of (1.1) and should be thought of as being the correct framework to deal with not necessarily unital algebras equipped with some fixed complete set of pairwise commuting idempotents (for example path algebras of not necessarily finite quivers). We therefore also introduce the notion of modules over graded categories which provides the usual definition of modules over an algebra under the correspondence (1.1) as explained in the Introduction. We denote

- by $\mathbb{k}$-Mod the category of all $\mathbb{k}$-vector spaces;

- by $\mathbb{k}$-mod the category of all finite-dimensional $\mathbb{k}$-vector spaces;

- by $\mathbb{k}$-gMod the category of all graded $\mathbb{k}$-vector spaces;

- by $\mathbb{k}$-gmod the category of all finite-dimensional graded $\mathbb{k}$-vector spaces;

- by $\mathbb{k}$-gfmod the category of all graded $\mathbb{k}$-vector spaces with finite-dimensional graded components. 
Let $\mathbf{C}$ be a graded category. A $\mathbb{k}$-linear functor $\mathrm{F}: \mathbf{C} \rightarrow \mathbb{k}$-gMod is called homogeneous of degree $d$ if it maps morphisms of degree $k$ to morphisms of degree $k+d$ for all $k \in \mathbb{Z}$. In particular, homogeneous functors of degree 0 preserve the degree of morphisms. A natural transformation between homogeneous functors is by definition grading preserving. We define

- the category $\mathbf{C}$-Mod of all $\mathbf{C}$-modules as the category of all $\mathbb{k}$-linear functors from $\mathbf{C}$ to $\mathbb{k}-$ Mod;

- the category $\mathbf{C}$-gMod of all graded $\mathbf{C}$-modules as the category of all $\mathbb{k}$-linear homogeneous functors of degree 0 from $\mathbf{C}$ to $\mathbb{k}$-gMod;

- the category $\mathbf{C}$-fmod of locally finite-dimensional $\mathbf{C}$-modules as the category of all $\mathbb{k}$-linear functors from $\mathbf{C}$ to $\mathbb{k}$-mod;

- the category $\mathbf{C}$-gfmod of locally finite-dimensional graded $\mathbf{C}$-modules as the category of all $\mathbb{k}$-linear homogeneous functors of degree 0 from $\mathbf{C}$ to $\mathbb{k}$-gfmod;

- the category $\mathbf{C}$-fdmod of finite-dimensional $\mathbf{C}$-modules as the category of all $\mathbb{k}$-linear functors from $\mathbf{C}$ to $\mathbb{k}$-mod satisfying the condition that the value of such functor is non-zero only on finitely many objects from $\mathbf{C}$;

- the category $\mathbf{C}$-gfdmod of finite-dimensional graded $\mathbf{C}$-modules as the category of all $\mathbb{k}$-linear homogeneous functors of degree 0 from $\mathbf{C}$ to $\mathbb{k}$-gmod satisfying the condition that the value of such functor is non-zero only on finitely many objects from $\mathbf{C}$.

Similarly, we define the corresponding categories of right $\mathbf{C}$-modules via the opposite category $\mathbf{C}^{\text {op }}$ (or, equivalently, using contravariant functors instead of covariant).

Graded modules over graded categories and modules over quotient categories. There is (see for example [CM, Section 2]) an equivalence of categories

$$
\mathrm{E}_{\mathbf{C}}: \quad \mathbf{C} \text {-gMod } \stackrel{\sim}{\longrightarrow} \mathbf{C}^{\mathbb{Z}} \text {-Mod }
$$

which is induced by the correspondence (2.1) and explicitly given as follows: For a graded $\mathbf{C}$-module, that is, a functor $\mathbf{M}: \mathbf{C} \rightarrow \mathbb{k}$-gMod, and for each object $(\lambda, i) \in \mathrm{Ob}\left(\mathbf{C}^{\mathbb{Z}}\right)$ we set $\mathrm{E}_{\mathbf{C}}(\mathrm{M})(\lambda, i)=\mathrm{M}(\lambda)_{i}$, where $\mathrm{M}(\lambda)=\bigoplus_{i \in \mathbb{Z}} \mathrm{M}(\lambda)_{i}$. For every $f \in \mathbf{C}^{\mathbb{Z}}((\lambda, i),(\mu, j))=\mathbf{C}_{j-i}(\lambda, \mu)$ we define $\mathrm{E}_{\mathbf{C}}(\mathrm{M})(f)=\mathrm{M}(f)_{i}$, which is a map from $\mathrm{E}_{\mathbf{C}}(\mathrm{M})(\lambda, i)=\mathrm{M}(\lambda)_{i}$ to $\mathrm{M}_{i+(j-i)}(\mu)=\mathrm{M}_{j}(\mu)=\mathrm{E}_{\mathbf{C}}(\mathrm{M})(\mu, j)$. This defines a functor, $\mathrm{E}_{\mathbf{C}}(\mathrm{M}): \mathbf{C}^{\mathbb{Z}} \rightarrow \mathbb{k}$-Mod, or, in other words, an object in $\mathbf{C}^{\mathbb{Z}}$-Mod. The assignment $\mathrm{M} \mapsto \mathrm{E}_{\mathbf{C}}(\mathrm{M})$ specifies what the functor $\mathrm{E}_{\mathbf{C}}$ does on the level of objects. If $\varphi: \mathrm{M} \rightarrow \mathrm{N}$ is a homomorphism of graded modules, for every $\lambda \in \operatorname{Ob}(\mathbf{C})$ and $i \in \mathbb{Z}$ we define $\mathrm{E}_{\mathbf{C}}(\varphi)(\lambda, i)$ to be $\varphi_{\lambda, i}: \mathrm{M}(\lambda)_{i} \rightarrow \mathrm{N}(\lambda)_{i}$ which is the restriction of the map $\varphi_{\lambda}$ to the the $i$-th graded component. This defines the functor $\mathrm{E}_{\mathbf{C}}$. For the inverse functor $\mathrm{E}_{\mathbf{C}}^{-1}$ and a $\mathbf{C}^{\mathbb{Z}}$-module $M$ we have $\mathrm{E}_{\mathbf{C}}^{-1}(M)(\lambda)=\bigoplus_{i \in \mathbb{Z}} M((\lambda, i))$ for any $\lambda \in \mathrm{Ob}(\mathbf{C})$. If $f \in \mathbf{C}(\lambda, \mu)$ is homogeneous of degree $j$, then for $i \in \mathbb{Z}$ we use the identification $\mathbf{C}_{j}(\lambda, \mu)=\mathbf{C}^{\mathbb{Z}}((\lambda, i),(\mu, j+i))$ to get the element $f(i)$, corresponding to $f$. Then we have $\mathrm{E}_{\mathbf{C}}^{-1}(M)(f)=\bigoplus_{i \in \mathbb{Z}} M(f(i))$. If $\varphi: M \rightarrow N$ is a natural transformation, we put $\left(\mathrm{E}_{\mathbf{C}}^{-1}(\varphi)\right)_{\lambda}=\bigoplus_{i \in \mathbb{Z}} \varphi_{\lambda, i}$. It is straightforward to check that these assignments define inverse equivalences of categories. For more details we refer the reader to [CM]. Obviously, the functor (2.2) restricts to an equivalence of categories

$$
\mathrm{E}_{\mathbf{C}}: \quad \mathbf{C} \text {-gfmod } \stackrel{\sim}{\longrightarrow} \mathbf{C}^{\mathbb{Z}} \text {-fmod. }
$$


Let $\mathbf{A}$ be a positively graded algebra and $\mathbf{A}$ the corresponding positively graded category. We define the following categories of A-modules and leave it as an exercise for the reader to check that they coincide with our previous definitions under the equivalence (1.1).

- A-Mod := A-Mod;

- A-gMod := A-gMod;

- A-mod as the category of all finitely-generated A-modules;

- A-gmod as the category of all finitely-generated graded A-modules;

- A-gfmod as the category of all graded A-modules with finite-dimensional graded components.

For a positively graded category $\mathbf{C}$ and $i \in \mathbb{Z}$ we denote by $\langle i\rangle: \mathbf{C}$-gMod $\rightarrow$ C-gMod the functor of shifting the grading, defined as follows: For objects $\lambda \in$ $\mathrm{Ob}(\mathbf{C})$ we have $\mathrm{M}\langle i\rangle(\lambda)_{j}=\mathrm{M}(\lambda)_{i+j}$ for all $j \in \mathbb{Z}$. On morphisms, the functor $\langle i\rangle$ is defined in the obvious (trivial) way.

For a positively graded category $\mathbf{C}$ the category $\mathbf{C}^{\text {op }}$ inherits a positive grading in the natural way, namely $\mathbf{C}_{i}^{\mathrm{op}}(\lambda, \mu)=\mathbf{C}_{i}(\mu, \lambda)$ for any $\lambda, \mu \in \mathbf{C}$, and $i \in \mathbb{Z}$. If $f \in \mathbf{C}_{i}^{\mathrm{op}}(\mu, \lambda)=\mathbf{C}_{i}(\lambda, \mu)$, and $g \in \mathbf{C}_{j}^{\mathrm{op}}(\nu, \mu)=\mathbf{C}_{i}(\mu, \nu)$, then $f \circ{ }^{\mathrm{op}} g=g \circ f \in$ $\mathbf{C}_{i+j}(\lambda, \nu)=\mathbf{C}_{i+j}^{\text {op }}(\nu, \lambda)$.

Bimodules, tensor products, Hom functors, and dualities. If $\mathcal{A}$ and $\mathcal{B}$ are two $\mathbb{k}$ linear categories, then an $\mathcal{A}$ - $\mathcal{B}$-bimodule is by definition an $\mathcal{A} \otimes_{\mathbb{k}} \mathcal{B}^{\mathrm{op}}$-module, where

$$
\begin{aligned}
\mathrm{Ob}\left(\mathcal{A} \otimes_{\mathbb{k}} \mathcal{B}^{\mathrm{op}}\right) & =\mathrm{Ob}(\mathcal{A}) \times \mathrm{Ob}\left(\mathcal{B}^{\mathrm{op}}\right), \\
\mathcal{A} \otimes_{\mathbb{k}} \mathcal{B}^{\mathrm{op}}\left((\lambda, \mu),\left(\lambda^{\prime}, \mu^{\prime}\right)\right) & =\mathcal{A}\left(\lambda, \lambda^{\prime}\right) \otimes_{\mathbb{k}} \mathcal{B}\left(\mu^{\prime}, \mu\right)
\end{aligned}
$$

for all $\lambda, \lambda^{\prime} \in \mathrm{Ob}(\mathcal{A})$ and $\mu, \mu^{\prime} \in \mathrm{Ob}(\mathcal{B})$.

Given an $\mathcal{B}^{\text {op }}$-module $X$ and a $\mathcal{B}$-module $Y$ we define the tensor product $X \otimes_{\mathcal{B}}$ $Y$ as the vector space $\bigoplus_{\lambda, \mu \in \mathcal{B}} X(\lambda) \otimes_{\mathbb{k}} Y(\mu)$ modulo the subspace $W$, which is generated by all the elements $X(b)(v) \otimes w-v \otimes Y(b)(w)$, where $v \in X(\lambda), w \in$ $Y(\mu)$ and $b \in \mathcal{B}(\mu, \lambda)=\mathcal{B}^{\mathrm{op}}(\lambda, \mu)$. If $X$ was an $\mathcal{A}$ - $\mathcal{B}$-bimodule, then the tensor product $X \otimes_{\mathcal{B}} Y$ is the $\mathcal{A}$-module, which assigns to $a \in \mathrm{Ob}(\mathcal{A})$ the vector space $X\left(a,,_{-}\right) \otimes_{\mathcal{B}} Y$ (and the obvious assignment on morphisms). One can easily check that this corresponds exactly to the usual tensor product of (bi)modules under the correspondence (1.1).

For two $\mathcal{A}$-modules $X$ and $Y$ the set $\mathcal{A}$ - $\operatorname{Mod}(X, Y)$ is obviously a vector space. If $X$ is an $\mathcal{A}$-B-bimodule, we define the $\mathcal{B}$-module $\mathcal{A}-\operatorname{Mod}(X, Y)$ in the following way: To any object $b$ from $\mathcal{B}$ we assign the vector space $\mathcal{A}-\operatorname{Mod}\left(X\left(_{-}, b\right), Y\right)$, and to each $f \in \mathcal{B}\left(b, b^{\prime}\right)$ we assign the map, which maps $g=\left(g_{a}\right)_{a \in \mathrm{Ob}(\mathcal{A})} \in \mathcal{A}-\operatorname{Mod}\left(X\left(_{-}, b\right), Y\right)$ to $h=\left(h_{a}\right)_{a \in \mathrm{Ob}(\mathcal{A})} \in \mathcal{A}-\operatorname{Mod}\left(X\left(_{-}, b^{\prime}\right), Y\right)$, where $h_{a}=g_{a} \circ X\left(e_{a}, f\right)$. Again, one checks that this corresponds exactly to the usual homomorphism construction under the correspondence (1.1).

It is straightforward to check that for a $\mathcal{B}$-module $Y$, an $\mathcal{A}$-module $Z$, and an $\mathcal{A}$-B-bimodule $X$ we have the usual functorial adjunction isomorphism

$$
\begin{aligned}
\mathcal{A}-\operatorname{Mod}\left(X \otimes_{\mathcal{B}} Y, Z\right) & \cong \mathcal{B}-\operatorname{Mod}(Y, \mathcal{A}-\operatorname{Mod}(X, Z)), \\
\varphi=\left\{\varphi_{a}\right\}_{a \in \operatorname{Ob}(\mathcal{A})} & \mapsto \hat{\varphi}=\left\{\hat{\varphi}_{b}\right\}_{b \in \operatorname{Ob}(\mathcal{B})}, \\
\check{\psi}=\left\{\check{\psi}_{a}\right\}_{a \in \mathrm{Ob}(\mathcal{A})} & \longleftrightarrow \psi=\left\{\psi_{b}\right\}_{b \in \mathrm{Ob}(\mathcal{B})}
\end{aligned}
$$

where for any $a \in \operatorname{Ob}(\mathcal{A}), b \in \mathrm{Ob}(\mathcal{B}), m \in Y(b)$ and $x \in X(a, b)$ we have $\hat{\varphi}_{b}(m)(x)=\varphi_{a}(x \otimes m) \in Z(a)$, and $\check{\psi}_{a}(x \otimes m)=\psi_{b}(x)(m) \in Z(a)$. 
Let $\mathbf{d}: \mathbb{k}-\operatorname{Mod} \rightarrow \mathbb{k}-\operatorname{Mod}$ be the usual duality functor $\mathbb{k}-\operatorname{Mod}(-, \mathbb{k})$. We also have the graded duality $\mathbb{D}: \mathbb{k}$-gMod $\rightarrow \mathbb{k}$-gMod for which $(\mathbb{D V})_{i}=\mathbf{d}\left(\mathrm{V}_{-i}\right)$ for any $\mathrm{V} \in \mathbb{k}$-gMod and which acts as the usual duality on morphisms. Note that if $\mathrm{M}$ is a graded $\mathbf{C}$-module, then $\mathbb{D M}$ (defined simply as a composition of functors) is a $\mathrm{C}^{\mathrm{op}}$-module.

2.3. The abelian category $\mathbf{C}$-gfmod. Let $\mathbf{C}$ be a positively graded $\mathbb{k}$-category. The following statement is obvious, but crucial:

Lemma 3. For any positively graded $\mathbb{k}$-category $\mathbf{C}$ the categories $\mathbf{C}$-gfmod and C-gmod are abelian categories.

Proof. The abelian structure is inherited from the abelian structure of $\mathbb{k}$-gfmod and $\mathbb{k}$-gmod. For details we refer to [Sc, p. 104].

Lemma 4. Let $\mathbf{C}$ be a positively graded category and $\lambda \in \operatorname{Ob}(\mathbf{C})$. Then $\mathrm{P}_{\mathbf{C}}(\lambda)=$ $\mathbf{C}\left(\lambda,,_{-}\right)$is an indecomposable projective object in both $\mathbf{C}$-gMod and $\mathbf{C}$-gfmod.

Proof. By definition we have $\mathrm{P}(\lambda)=\mathrm{P}_{\mathbf{C}}(\lambda)=\mathbf{C}\left(\lambda,,_{-}\right) \in \mathbf{C}$-gMod. Because of the assumption (C-iii), it is even an object of $\mathbf{C}$-gfmod. It is indecomposable, since the only non-trivial idempotent of its endomorphism ring is the identity (by the assumption (C-(iii)) and using the Yoneda lemma). To see that it is projective, let $\varphi: \mathrm{F} \rightarrow \mathrm{G}$ and $\alpha: \mathrm{P}(\lambda) \rightarrow \mathrm{G}$ be morphisms between graded $\mathrm{C}$-modules, where $\varphi$ is surjective. We have to show that there is a morphism $\Phi: \mathrm{P}(\lambda) \rightarrow \mathrm{F}$ such that $\varphi \circ \Phi=\alpha$. Choose $b \in \varphi^{-1}\left(\alpha\left(e_{\lambda}\right)\right) \subset \mathrm{F}(\lambda)$ and define $\Phi(f)=\mathrm{F}(f)(b)$ for any $f \in \mathrm{P}(\lambda)(\mu)=\mathbf{C}(\lambda, \mu)$. Then we have

$$
\varphi(\Phi(f))=(\varphi \circ \mathrm{F}(f))(b)=(\mathrm{G}(f) \circ \varphi)(b)=\mathrm{G}(f)(\alpha(e))=\alpha(f e)=\alpha(f) .
$$

Hence, $\mathrm{P}_{\mathbf{C}}(\lambda)$ is projective, and we are done.

Factoring out the unique maximal graded submodule of $\mathrm{P}(\lambda)$, that is, the submodule given by all elements of positive degree, we obtain the graded simple module $\mathrm{L}(\lambda)$. The duality $\mathbb{D}$ maps projective objects to injective objects and preserves indecomposability, hence we have the graded indecomposable injective envelope $\mathrm{I}(\lambda)=\mathbb{D} \mathbf{C}^{\mathrm{op}}\left(\lambda,,_{-}\right)$of $\mathrm{L}(\lambda)$. If we forget the grading, we obtain the ungraded $\mathbf{C}$ modules $P(\lambda), L(\lambda)$ and $I(\lambda)$, respectively. Note that they are still indecomposable, and, of course, $L(\lambda)$ is simple. We define

$$
\begin{aligned}
& \mathrm{P}=\mathrm{P}_{\mathbf{C}}=\bigoplus_{\lambda \in \mathrm{Ob}(\mathbf{C})} \mathrm{P}_{\mathbf{C}}(\lambda), \\
& \mathrm{I}=\mathrm{I}_{\mathbf{C}}=\bigoplus_{\lambda \in \operatorname{Ob}(\mathbf{C})} \mathrm{I}_{\mathbf{C}}(\lambda), \\
& \mathrm{L}=\mathrm{L}_{\mathbf{C}}=\bigoplus_{\lambda \in \operatorname{Ob}(\mathbf{C})} \mathrm{L}_{\mathbf{C}}(\lambda) .
\end{aligned}
$$

Lemma 5. Let $\mathbf{C}$ be a positively graded category, without necessarily satisfying (C iv). The simple objects in $\mathbf{C}$-gfmod are exactly the modules of the form $\mathrm{L}(\lambda)\langle i\rangle$, $\lambda \in \mathbf{C}, i \in \mathbb{Z}$. Any object in $\mathbf{C}$-gfmod of finite length has a projective cover and an injective hull.

Proof. From Lemma 3 we know that $\mathbf{C}$-gfmod is an abelian category. Let $\mathbf{M} \in$ C-gfmod be simple. Let $0 \neq v \in \mathrm{M}(\lambda)_{i}$ for some $\lambda, i$. Then there is a non-trivial, hence a surjective, morphism $\mathrm{P}(\lambda)\langle-i\rangle \rightarrow \mathrm{M}$ sending $e_{\lambda}$ to $v$. From the positivity of the grading we get $\mathrm{M} \cong \mathrm{L}(\lambda)\langle-i\rangle$. 
The assumption ( $\mathrm{C}$-iv $)$ in Definition 1 was introduced in order to have the following result available:

Lemma 6. Let $\mathbf{C}$ be a positively graded category.

(a) Let $\mathrm{M}$ be an object in $\mathbf{C}$-gfmod. Assume there exist some $k \in \mathbb{Z}$ with the following property: If $j<k$, then $\mathrm{M}(\mu)_{j}=\{0\}$ for any $\mu \in \mathrm{Ob}(\mathbf{C})$. Then $\mathrm{M}$ has a projective cover in $\mathbf{C}$-gfmod.

(b) In particular, any simple object $\mathrm{L}(\lambda)$ has a minimal projective resolution.

(c) Dually, any simple object $\mathrm{L}(\lambda)$ has a minimal injective coresolution.

Proof. Let $\mathcal{A}$ denote the full subcategory of $\mathbf{C}$-gfmod, which consists of all modules satisfying the conditions of statement (国). Statement (国) would follow from the general theory of projective covers (see for example [Sh, Proposition 1]), provided that we prove two things. Namely, that each object from $\mathcal{A}$ is a quotient of some projective object from $\mathbf{C}$-gfmod, and that for any epimorphism $f: \mathrm{X} \rightarrow \mathrm{Y}$ in $\mathcal{A}$ there is a minimal submodule $\mathrm{Z}$ of $\mathrm{X}$ with respect to the condition $f(\mathrm{Z})=\mathrm{Y}$.

First we prove that each object from $\mathcal{A}$ is a quotient of some projective object from $\mathbf{C}$-gfmod Let $M=\mathrm{E}_{\mathbf{C}}(\mathrm{M}) \in \mathbf{C}^{\mathbb{Z}}$-fmod. Define $N \in \mathbf{C}^{\mathbb{Z}}$-Mod as follows:

$$
N=\bigoplus_{\mu \in \mathrm{Ob}(\mathbf{C}), r \in \mathbb{Z}} \bigoplus_{s=1}^{\operatorname{dim}_{\mathrm{k}} M((\mu, r))} \mathbf{C}^{\mathbb{Z}}\left((\mu, r),_{-}\right)
$$

Note that the second sum just indicates that we take a certain number of copies of $\mathbf{C}\left((\mu, r),_{-}\right)$. Since $\mathbf{M} \in \mathbf{C}$-gfmod $\cong \mathbf{C}^{\mathbb{Z}}$-fmod, the space $M(\mu, r)$ is always finite-dimensional, hence the second sum of (2.5) is finite. By the assumption on $\mathbf{M}$ it is enough to take $r \geq k$. Since $\mathbf{C}$ is positively graded, we can have $\mathbf{C}((\mu, r),(\lambda, i))=\mathbf{C}(\mu, \lambda)_{i-r} \neq\{0\}$ only if $i-r \geq 0$, that is, $r \leq i$. Hence we get

$$
N((\lambda, i))=\bigoplus_{r=k}^{i} \bigoplus_{\mu \in \mathrm{Ob}(\mathbf{C})} \bigoplus_{s=1}^{\operatorname{dim}_{\mathrm{k}} M((\mu, r))} \mathbf{C}((\mu, r),(\lambda, i)) .
$$

Because of condition (C-iv), the second sum appearing in (2.6) in fact produces only a finite number of non-zero summands. Hence $N((\lambda, i))$ is finite-dimensional, so $N \in \mathbf{C}^{\mathbb{Z}}$-fmod $\cong \mathbf{C}$-gfmod. By construction, $N$ is projective and surjects onto $M$.

The fact that for any epimorphism $f: \mathrm{X} \rightarrow \mathrm{Y}$ in $\mathcal{A}$ there is a minimal submodule $\mathrm{Z}$ of $\mathrm{X}$ with respect to the condition $f(\mathrm{Z})=\mathrm{Y}$ follows from the definition of $\mathcal{A}$ using the standard arguments involving Zorn's lemma. Hence statement (a) now follows [Sh, Proposition 1].

The second statement of the lemma follows from the first one and from the remark that the condition on $\mathrm{M}$ in (国) is also satisfied for the kernel of the projective cover of M, constructed above. The last statement follows by duality.

For $\lambda \in \operatorname{Ob}(\mathbf{C})$ let $\mathcal{Q}_{\lambda}^{\bullet}$ and $\mathcal{J}_{\lambda}^{\bullet}$ denote a fixed minimal projective resolution and a minimal injective coresolution of $\mathrm{L}(\lambda)$ in $\mathbf{C}$-gfmod, respectively. It is easy to check that such (co)resolutions are unique up to isomorphism. It is not difficult to see that $\mathbf{C}$-gMod has enough projectives, whereas $\mathbf{C}$-gfmod $\cong \mathbf{C}^{\mathbb{Z}}$-fmod does not need to have enough projectives in general (see the example of the quivers (1.3) and (1.6) ). 


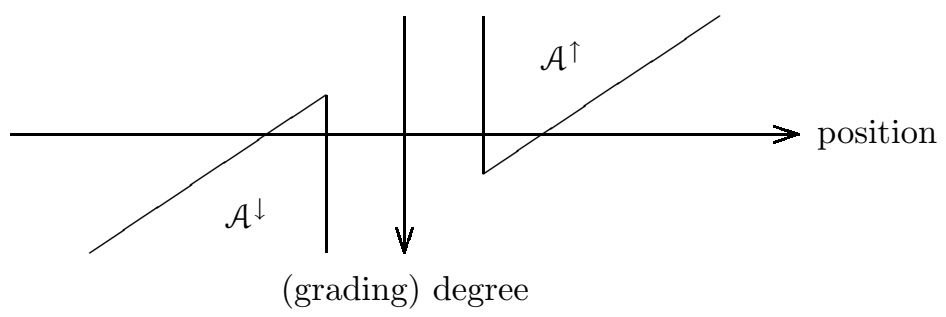

Figure 1. The supports of objects from the categories $\mathcal{A}^{\downarrow}$ and $\mathcal{A}^{\uparrow}$.

2.4. Some general notation. In the following we will sometimes write $M_{\mathbf{C}}$ to indicate that $M$ is a (left!) $\mathbf{C}$-module. If $\mathcal{X}^{\bullet}$ is a complex of modules with differential $d^{\bullet}$, then $d^{i}: \mathcal{X}^{i} \rightarrow \mathcal{X}^{i+1}$ for all $i \in \mathbb{Z}$. If $M$ is a module, $M^{\bullet}$ will denote the complex where $M^{i}=0, i \neq 0$, and $M^{0}=M$ with the trivial differential. For $i \in \mathbb{Z}$ we denote by $[i]$ the functor of shifting the position in a complex, defined for any complex $\mathcal{X}^{\bullet}$ as follows: $\mathcal{X}[i]^{j}=\mathcal{X}^{i+j}$ for all $j \in \mathbb{Z}$. We denote by $\mathcal{H}^{i} \mathcal{X} \bullet$ the $i$-th cohomology of $\mathcal{X}^{\bullet}$. In the hope to avoid confusion we will use the word degree for the degree in the grading, and the word position for the degree in a complex. An example: If a graded module $\mathbf{M}$ is concentrated in degree 0 , then $\mathbf{M}^{\bullet}[i]\langle j\rangle$ is concentrated in position $-i$ and degree $-j$.

For an abelian category, $\mathcal{A}$, we denote by $\mathcal{C}(\mathcal{A})$ the category of complexes of objects from $\mathcal{A}$, by $\mathcal{K}(\mathcal{A})$ its homotopy category, and by $\mathcal{D}(\mathcal{A})$ the corresponding derived category. We will use the standard upper indices $b,+$, and - to denote the corresponding categories of bounded, right bounded and left bounded complexes. If $\mathcal{A}$ has enough projectives and $\mathrm{F}: \mathcal{A} \rightarrow \mathcal{A}$ is a right exact functor, we denote by $\mathcal{L} \mathrm{F}$ its left derived functor and by $\mathcal{L}_{i} \mathrm{~F}$ the $i$-th cohomology functor of $\mathrm{F}$. Analogously we define $\mathcal{R} F$ and $\mathcal{R}^{i} \mathrm{~F}$, if $\mathrm{F}$ is left exact and $\mathcal{A}$ has enough injectives. The symbol ID denotes the identity functor.

For a graded vector space, $V=\bigoplus_{i \in \mathbb{Z}} V_{i}$, and for $j \in \mathbb{Z}$ we denote by $\operatorname{Lev}_{j}$ the operation of taking the $j$-th graded component of $V$, that is, $\operatorname{Lev}_{j}(V)=V_{j}$.

Let $\mathcal{A}$ be an abelian category whose objects are some graded modules. Following BGS, 2.12], we denote by $\mathcal{C}^{\downarrow}(\mathcal{A})$ the category of complexes of graded modules from $\mathcal{A}$, which consists of all complexes $\mathcal{X}^{\bullet} \in \mathcal{A}$, such that there exist integers $N_{1}\left(\mathcal{X}^{\bullet}\right)$ and $N_{2}\left(\mathcal{X}^{\bullet}\right)$ satisfying

$$
\operatorname{Lev}_{j}\left(\mathcal{X}^{i}\right)=0 \quad \text { for all } i>N_{1}\left(\mathcal{X}^{\bullet}\right) \quad \text { and all } i+j<N_{2}\left(\mathcal{X}^{\bullet}\right) ;
$$

and by $\mathcal{C}^{\uparrow}(\mathcal{A})$ the category of complexes of graded modules from $\mathcal{A}$, such that there exist integers $N_{1}\left(\mathcal{X}^{\bullet}\right)$ and $N_{2}\left(\mathcal{X}^{\bullet}\right)$ satisfying

$$
\operatorname{Lev}_{j}\left(\mathcal{X}^{i}\right)=0 \text { for all } i<N_{1}\left(\mathcal{X}^{\bullet}\right) \text { and all } i+j>N_{2}\left(\mathcal{X}^{\bullet}\right) \text {. }
$$

Thus the non-zero components of the objects from $\mathcal{C}^{\downarrow}(\mathcal{A})$ and $\mathcal{C}^{\uparrow}(\mathcal{A})$ are concentrated in regions as depicted in Figure 1. We further denote by $\mathcal{K}^{\downarrow}(\mathcal{A}), \mathcal{K}^{\uparrow}(\mathcal{A})$, $\mathcal{D}^{\downarrow}(\mathcal{A})$, and $\mathcal{D}^{\uparrow}(\mathcal{A})$ the corresponding homotopy and derived categories. Our notation is exactly opposite to the one in [BGS, 2.12]. We made this change, since we think our choice is better adjusted to the usual terminology that an indecomposable projective module has a simple head (or top), which in our picture indeed corresponds to the highest part of a depicted module. 
For a complex $\mathcal{X} \bullet$ and $i \in \mathbb{Z}$ we denote by $\left(\mathfrak{t}_{i} \mathcal{X}\right)^{\bullet}$ the naively $i$-truncated complex, defined as follows: $\left(\mathfrak{t}_{i} \mathcal{X}\right)^{j}=\mathcal{X}^{j}$ for all $j \leq i$, and $\left(\mathfrak{t}_{i} \mathcal{X}\right)^{j}=0$ for all $j>i$, with the differential on $\left(\mathfrak{t}_{i} \mathcal{X}\right)^{\bullet}$ induced from that on $\mathcal{X}^{\bullet}$.

\section{Categories of linear complexes}

In this section we will introduce one of the main players, the category of linear complexes (usually of projective modules) as they appear for example in [MVS. For Koszul algebras the categories of linear complexes already appeared in BGS, Corollary 2.13.3] (as cores of non-standard $t$-structure). Let $\mathrm{M}$ be a graded-structures $\mathrm{C}$-module. We denote by $\mathcal{L} \mathcal{C}(\mathrm{M})$ the category of linear complexes associated with $\mathrm{M}$, which is defined as follows: The objects of $\mathcal{L} \mathcal{C}(\mathrm{M})$ are all complexes $\mathcal{X}^{\bullet}$ such that for every $i \in \mathbb{Z}$ every indecomposable summand of the module $\mathcal{X}^{i}$ occurs with finite multiplicity and has the form $\mathrm{N}\langle i\rangle$, where $N$ is an indecomposable summand of $M$; the morphisms in $\mathcal{L} \mathcal{C}(\mathrm{M})$ are all possible morphisms of complexes of graded modules. In the special case when $\mathrm{M}=\mathrm{P}$ (as defined in (2.4)), the category $\mathcal{L} \mathcal{C}(\mathrm{M})=\mathcal{L} \mathcal{C}(\mathrm{P})$ is called the category of linear complexes of projective modules. In the case $\mathrm{M}=\mathrm{I}$, the category $\mathcal{L} \mathcal{C}(\mathrm{M})=\mathcal{L} \mathcal{C}(\mathrm{I})$ is called the category of linear complexes of injective modules.

For $k \in \mathbb{Z}$ let $\mathcal{L} \mathcal{C}(\mathrm{P})^{\geq k}$ be the full subcategory of $\mathcal{L} \mathcal{C}(\mathrm{P})$ given by all complexes $\mathrm{M}$ satisfying $\mathrm{M}^{j}=\{0\}$ for $j<k$. Obviously, $\mathcal{L} \mathcal{C}(\mathrm{P})=\lim \mathcal{L} \mathcal{C}(\mathrm{P})^{\geq k}$, where the inverse system is given by truncation functors. Let us recall some basic facts about the categories of linear complexes:

Proposition 7. (i) Both $\mathcal{L} \mathcal{C}(\mathrm{P})$ and $\mathcal{L} \mathcal{C}(\mathrm{I})$ are abelian categories with the usual kernels and cokernels for complexes.

(ii) The simple objects of $\mathcal{L} \mathcal{C}(\mathrm{P})$ (resp. $\mathcal{L} \mathcal{C}(\mathrm{I})$ ) are exactly the complexes of the form $\mathrm{P}(\lambda)^{\bullet}\langle-i\rangle[i]$ (resp. $\mathrm{I}(\lambda)^{\bullet}\langle-i\rangle[i]$ ), where $\lambda \in \mathrm{Ob}(\mathbf{C})$ and $i \in \mathbb{Z}$.

(iii) The Nakayama functor $\mathrm{N}=\mathrm{N}_{\mathbf{C}}=\left(\mathbb{D} \mathbf{C}\left({ }_{-},{ }_{-}\right)\right) \otimes_{\mathbf{C}}$ - induces an equivalence between $\mathcal{L} \mathrm{C}(\mathrm{P})$ and $\mathcal{L} \mathrm{C}(\mathrm{I})$. The Nakayama functor satisfies

$$
\mathrm{N}(\mathrm{P}(\lambda)\langle i\rangle[-i]) \cong \mathrm{N}(\mathrm{P}(\lambda))\langle i\rangle[-i] \cong \mathrm{I}(\lambda)\langle i\rangle[-i]
$$

for any $\lambda \in \mathrm{Ob}(\mathbf{C})$ and $i \in \mathbb{Z}$.

Proof. The statements (ii) and (iii) are proved in [MO, Lemma 5]. The existence of the equivalence from part (iiii) follows from the standard fact that $\mathrm{N}$ induces an equivalence between the additive closures of $\mathrm{P}$ and $\mathrm{I}$ with finite multiplicities (see for example [Ha, I.4.6]). The formulas hold by definition.

3.1. Projective and injective objects in $\mathcal{L} \mathcal{C}(\mathrm{P})$. The purpose of this section is to give an explicit constructible description of the indecomposable projective covers and injective hulls of simple objects in the category $\mathcal{L} \mathcal{C}(\mathrm{P})$. These projective and injective objects exist, although the category does not have enough projectives or enough injectives in general. The analogous results for $\mathcal{L} \mathcal{C}(\mathrm{I})$ can be obtained by applying the Nakayama automorphism from Proposition 7 . Recall that for $\lambda \in \mathrm{Ob}(\mathbf{C})$ we denote by $\mathcal{Q}_{\lambda}^{\bullet}$ (and $\mathcal{J}_{\dot{\lambda}}^{\bullet}$ respectively) a fixed minimal projective resolution (and a fixed minimal injective coresolution) of $L(\lambda)$, considered as an object of $\mathbf{C}$-gfmod (see Lemma 6). We will show in Proposition 111 below how injective (respectively, projective) objects in $\mathcal{L} \mathcal{C}(\mathrm{P})$ can be considered as maximal linear parts of the $\mathcal{Q}_{\lambda}^{\bullet}$ 's 
(respectively, of the images under the inverse Nakayama functor applied to the $\mathcal{J}_{\lambda}^{\bullet}$ 's). We start with some preparation.

We will call a complex minimal provided that it does not contain any direct summands of the form

$$
\mathrm{N} \rightarrow 0 \rightarrow \mathrm{M} \stackrel{\sim}{\rightarrow} \mathrm{M} \rightarrow 0 \rightarrow \ldots
$$

Consider the full subcategory $\overline{\mathcal{C}}(\mathbf{C})$ of the category of complexes of graded $\mathbf{C}$ modules, whose objects are all possible minimal complexes $\mathcal{X}^{\bullet}$ such that for every $j \in \mathbb{Z}$ every indecomposable direct summand of $\mathcal{X}^{j}$ is isomorphic to $\mathrm{P}(\lambda)\langle k\rangle$ for some $\lambda \in \operatorname{Ob}(\mathbf{C})$ and some $k \in \mathbb{Z}$. Denote by $\overline{\mathcal{K}}(\mathbf{C})$ the corresponding homotopy category.

Fix for the moment $i \in \mathbb{Z}$ and let $\mathcal{X}^{\bullet} \in \overline{\mathcal{K}}(\mathbf{C})$ with the differential $d^{\bullet}$. For every $j \in \mathbb{Z}$ we have the following canonical decomposition of $\mathcal{X}^{j}$ :

$$
\mathcal{X}^{j}=\mathcal{X}\{>i\}^{j} \oplus \mathcal{X}\{=i\}^{j} \oplus \mathcal{X}\{<i\}^{j},
$$

where all the indecomposable direct summands

of $\mathcal{X}\{>i\}^{j}$ are isomorphic to $\mathrm{P}(\lambda)\langle k\rangle$ for some $\lambda \in \mathrm{Ob}(\mathbf{C})$ and $k>i$, of $\mathcal{X}\{=i\}^{j}$ are isomorphic to $\mathrm{P}(\lambda)\langle i\rangle$ for some $\lambda \in \mathrm{Ob}(\mathbf{C})$, of $\mathcal{X}\{<i\}^{j}$ are isomorphic to $\mathrm{P}(\lambda)\langle k\rangle$ for some $\lambda \in \mathrm{Ob}(\mathbf{C})$ and $k<i$.

Lemma 8. Let $\mathcal{X} \bullet \in \overline{\mathcal{K}}(\mathbf{C})$. For any $i, j \in \mathbb{Z}$ we have the following inclusion: $d^{j}\left(\mathcal{X}\{>i\}^{j}\right) \subset \mathcal{X}\{>i+1\}^{j+1}$.

Proof. We have of course $d^{j}\left(\mathcal{X}^{j}\right) \subset \mathcal{X}^{j+1}$. Now let $\mathrm{P}(\lambda)^{\bullet}\langle k\rangle$ be a summand of $\mathcal{X}\{>i\}^{j}$, that is, $k>i$. Let $\mathrm{P}(\mu)^{\bullet}\langle l\rangle$ be a summand of $\mathcal{X}^{j+1}$ such that $d^{j}$ induces a non-trivial morphism

$$
\alpha \in \mathbf{C}-\operatorname{gMod}(\mathrm{P}(\lambda)\langle k\rangle, \mathrm{P}(\mu)\langle l\rangle)=\mathbf{C}-\operatorname{Mod}(\mathrm{P}(\lambda), \mathrm{P}(\mu))_{l-k}=\mathbf{C}(\mu, \lambda)_{l-k} .
$$

Since $\mathbf{C}$ is positively graded, we have $l \geq k$, hence

$$
d^{j}\left(\mathcal{X}\{>i\}^{j}\right) \subseteq \mathcal{X}\{>i\}^{j+1}=\mathcal{X}\{>i+1\}^{j+1} \oplus \mathcal{X}\{=i+1\}^{j+1} .
$$

The positivity of the grading also implies that the only indecomposable direct summands of $\mathcal{X}\{>i\}^{j}$ which can be mapped to $\mathcal{X}\{=i+1\}^{j+1}$ are the ones isomorphic to $\mathrm{P}(\lambda)\langle i+1\rangle$ for some $\lambda \in \mathrm{Ob}(\mathbf{C})$, in which case the corresponding map must be an isomorphism. This is impossible because of the minimality of $\mathcal{X}^{\bullet}$. The claim follows.

Lemma 8 allows us to define, depending on some fixed $i \in \mathbb{Z}$, the following functor (which picks out the part "supported above the $i$-shifted diagonal"):

$$
\begin{aligned}
\mathrm{S}_{i}=\mathrm{S}_{i}^{\mathbf{C}}: \overline{\mathcal{K}}(\mathbf{C}) & \rightarrow \overline{\mathcal{K}}(\mathbf{C}), \\
\mathcal{X}^{\bullet} & \mapsto \mathcal{X}\{>i+\bullet\} \bullet
\end{aligned}
$$

where the differential on $\mathcal{X}\{>i+\bullet\} \bullet$ is induced from that on $\mathcal{X} \bullet$ by restriction. By definition, there is a natural inclusion of functors $\mathrm{S}_{i} \hookrightarrow \mathrm{ID}$. We denote by $\mathrm{Q}_{i}=\mathrm{Q}_{i}^{\mathbf{C}}$ the quotient functor.

Lemma 9. Let $\mathcal{X}^{\bullet} \in \overline{\mathcal{K}}(\mathbf{C})$ be such that for every $j \in \mathbb{Z}$ each indecomposable summand of $\mathcal{X}^{j}$ occurs with finite multiplicity. Then $\mathrm{S}_{-1} \mathrm{Q}_{0} \mathcal{X} \bullet$ is a linear complex of projectives, hence an object in $\mathcal{L} \mathcal{C}(\mathrm{P})$. 
Proof. The statement follows directly from the definitions, because, at the position $j$, the functor $\mathrm{S}_{-1} \mathrm{Q}_{0} \mathcal{X}^{\bullet}$ picks out the summands of the form $\mathrm{P}(\lambda)\langle k\rangle$, where $\lambda \in$ $\mathrm{Ob}(\mathbf{C})$ and $j-1<k \leq j$.

Note that the functor $\mathrm{S}_{-1} \mathrm{Q}_{0}$ is exactly picking out the (maximal) linear part of a complex. Denote by $\mathcal{K}_{\mathbf{C}}^{\vee}$ the full subcategory of $\overline{\mathcal{K}}(\mathbf{C})$, which consists of all complexes $\mathcal{X}^{\bullet} \in \overline{\mathcal{K}}(\mathbf{C})$, such that each indecomposable direct summand occurs with finite multiplicity in $\mathcal{X}^{j}$ for any $j$, and $\mathbf{C}-\operatorname{Mod}\left(\mathcal{X}^{i}, \mathrm{~L}\langle j\rangle\right) \neq 0$ implies $j \leq i$ for all $i, j \in \mathbb{Z}$. Then we have the natural inclusion

$$
\text { incl }: \mathcal{L} \mathcal{C}(\mathrm{P}) \rightarrow \mathcal{K}_{\mathbf{C}}^{\vee}
$$

Lemma 10. The functor $\mathrm{S}_{-1} \mathrm{Q}_{0}: \mathcal{K}_{\mathbf{C}}^{\vee} \rightarrow \mathcal{L} \mathcal{C}(\mathrm{P})$ is right adjoint to incl.

Proof. Let $\mathcal{X}^{\bullet} \in \mathcal{L} \mathcal{C}(\mathrm{P})$ and $\mathcal{Y}^{\bullet} \in \mathcal{K}_{\mathbf{C}}^{\vee}$. Since $\mathbf{C}$ is positively graded, using the same arguments as in the proof of Lemma 8 , we have

$$
\overline{\mathcal{K}}(\mathbf{C})\left(\operatorname{incl} \mathcal{X}^{\bullet}, \mathcal{Y}^{\bullet}\right) \cong \overline{\mathcal{K}}(\mathbf{C})\left(\operatorname{incl} \mathcal{X}^{\bullet}, \mathrm{S}_{-1} \mathrm{Q}_{0} \mathcal{Y}^{\bullet}\right) \stackrel{(\operatorname{Lemma} 9}{\cong} \mathcal{L}(\mathrm{P})\left(\mathcal{X}^{\bullet}, \mathrm{S}_{-1} \mathrm{Q}_{0} \mathcal{Y}^{\bullet}\right)
$$

The claim follows.

Proposition 11. Let $\lambda \in \mathrm{Ob}(\mathbf{C})$.

(a) The simple object $\mathrm{P}(\lambda)^{\bullet}$ of $\mathcal{L} \mathcal{C}(\mathrm{P})$ has a projective cover $\mathcal{P}_{\lambda}^{\bullet}$ and an injective hull $\mathcal{I}_{\lambda}$. Hence, any simple object in $\mathcal{L} \mathfrak{C}(\mathrm{P})$ has a projective cover and an injective hull.

(b) There are isomorphisms, of objects from $\mathcal{L} \mathcal{C}(\mathrm{P})$, as follows:

(i) $\mathcal{I}_{\lambda}^{\bullet} \cong \mathrm{S}_{-1} \mathrm{Q}_{0} \mathcal{Q}_{\lambda}^{\bullet}$.

(ii) $\mathcal{P}_{\dot{\lambda}} \cong \mathrm{S}_{-1} \mathrm{Q}_{0} \mathrm{~N}^{-1} \mathcal{J}_{\lambda}$, where $\mathrm{N}$ is the Nakayama functor from Proposition 7 .

Since it is quite easy to prove Proposition 11(b) assuming the existence of the projective covers and injective hulls as claimed, we will first give a separate proof for this part. In this proof we will compare the functors $\mathcal{L} \mathcal{C}(\mathrm{P})\left({ }_{-}, \mathcal{I}_{\lambda}^{\bullet}\right)$ and $\mathcal{L} \mathcal{C}(\mathrm{P})\left({ }_{-}, \mathrm{S}_{-1} \mathrm{Q}_{0} \mathcal{Q}_{\lambda}^{\bullet}\right)$ and show that they are isomorphic. The second proof is more technical, but provides the existence as well. It characterizes $\mathrm{S}_{-1} \mathrm{Q}_{0} \mathcal{Q}_{\lambda}^{\bullet}$ as the unique object having simple socle $\mathrm{P}(\lambda)^{\bullet}$ and being injective.

Proof of Proposition 11(b) assuming the existence part (国). Consider the functors

$$
\mathrm{F}_{1}:=\mathcal{L} \mathcal{C}(\mathrm{P})\left({ }_{-}, \mathcal{I}_{\lambda}^{\bullet}\right), \quad \mathrm{F}_{2}:=\mathcal{L} \mathcal{C}(\mathrm{P})\left({ }_{-}, \mathrm{S}_{-1} \mathrm{Q}_{0} \mathcal{Q}_{\lambda}^{\bullet}\right) .
$$

Since $\mathcal{I}_{\lambda}^{\bullet}$ is the injective hull of the simple object $\mathrm{P}(\lambda)^{\bullet}$ in $\mathcal{L} \mathcal{C}(\mathrm{P})$, we have for any $\mathcal{X} \bullet \mathcal{L} \mathcal{C}(\mathrm{P})$ the isomorphism

$$
\mathrm{F}_{1}\left(\mathcal{X}^{\bullet}\right) \cong \mathbf{C}-\operatorname{gfmod}\left(\mathcal{X}^{0}, \mathrm{P}(\lambda)\right) \cong \mathbf{d}\left(\operatorname{Lev}_{0}\left(\mathcal{X}^{0}(\lambda)\right)\right) \text {. }
$$

On the other hand, since $\mathcal{X}^{\bullet}$ is a linear complex of projective modules, applying Lemma 10 we have

$$
\mathrm{F}_{2}\left(\mathcal{X}^{\bullet}\right) \cong \mathcal{K}_{\mathbf{C}}^{\vee}\left(\operatorname{incl} \mathcal{X}^{\bullet}, \mathcal{Q}_{\lambda}^{\bullet}\right) \cong \mathcal{K}(\mathbf{C} \text {-gfmod })\left(\operatorname{incl} \mathcal{X}^{\bullet}, \mathrm{L}(\lambda)^{\bullet}\right) \cong \mathbf{d}\left(\operatorname{Lev}_{0}\left(\mathcal{X}^{0}(\lambda)\right)\right)
$$

Since all the isomorphisms are natural, it follows that the functors $F_{1}$ and $F_{2}$ are isomorphic. Therefore, there must be an isomorphism $\mathcal{I}_{\lambda}^{\bullet} \cong \mathrm{S}_{-1} \mathrm{Q}_{0} \mathcal{Q}_{\lambda}^{\bullet}$. The second statement then follows by applying Proposition 7. 
Proof of Proposition 11 including the existence. We first note that the implication in part (a) is clear, since if $\mathcal{P}_{\lambda}^{\bullet}$ is a projective cover and $\mathcal{I}_{\dot{\lambda}}^{\bullet}$ is an injective hull of the simple object $\mathrm{P}(\lambda)^{\bullet}$, then $\mathcal{P}_{\lambda}^{\bullet}\langle-i\rangle[i]$ is a projective cover and $\mathcal{I}_{\lambda}^{\bullet}\langle-i\rangle[i]$ is an injective hull of the simple object $\mathrm{P}(\lambda)^{\bullet}\langle-i\rangle[i]$; we are done by Proposition 7 .

Set $\mathcal{X} \bullet=\mathrm{S}_{-1} \mathrm{Q}_{0} \mathcal{Q}_{\lambda}^{\bullet}$. This is an object of $\mathcal{L} \mathcal{C}(\mathrm{P})$ by Lemma 9, Using Lemma 10, we calculate

$$
\begin{aligned}
\mathcal{L} \mathcal{C}(\mathrm{P})\left(\mathrm{P}(\mu)^{\bullet}\langle-i\rangle[i], \mathcal{X}^{\bullet}\right) & =\mathcal{L} \mathcal{C}(\mathrm{P})\left(\mathrm{P}(\mu)^{\bullet}\langle-i\rangle[i], \mathrm{S}_{-1} \mathrm{Q}_{0} \mathcal{Q}_{\lambda}^{\bullet}\right) \\
& \cong \mathcal{K}_{\mathbf{C}}^{\vee}\left(\operatorname{inclP}(\mu)^{\bullet}\langle-i\rangle[i], \mathcal{Q}_{\lambda}^{\bullet}\right) .
\end{aligned}
$$

From the definition of $\mathcal{Q}_{\lambda}$ we therefore get the following: For $\mu \in \mathrm{Ob}(\mathbf{C})$ and $i \in \mathbb{Z}$ we have

$$
\mathcal{L} \mathcal{C}(\mathrm{P})\left(\mathrm{P}(\mu)^{\bullet}\langle-i\rangle[i], \mathcal{X}^{\bullet}\right)= \begin{cases}\mathbb{k}, & \text { if } \mu=\lambda, i=0, \\ 0, & \text { otherwise }\end{cases}
$$

This implies that $\mathcal{X}^{\bullet}$ has, as an object of $\mathcal{L} \mathcal{C}(\mathrm{P})$, simple socle, namely $\mathrm{P}(\lambda)^{\bullet}$. Thus, to complete the proof we just have to show that $\mathcal{X} \bullet$ is an injective object of $\mathcal{L} \mathcal{C}(\mathrm{P})$. We claim that it is even enough to show that

$$
\operatorname{Ext}_{\mathcal{L} \mathcal{C}(\mathrm{P})}^{1}\left(\mathrm{P}(\mu)\langle-i\rangle[i], \mathcal{X}^{\bullet}\right)=0
$$

for all $\mu \in \operatorname{Ob}(\mathbf{C})$ and $i \in \mathbb{Z}$. Indeed, if we fix $k \in \mathbb{Z}$, then formula (3.1) implies that $\operatorname{Ext}_{\mathcal{L} \mathfrak{C}(\mathrm{P})}^{1}\left(\mathcal{Y}^{\bullet}, \mathcal{X}^{\bullet}\right)=0$ for any $\mathcal{Y}^{\bullet} \in \mathcal{L} \mathcal{C}(\mathrm{P})^{\geq k}$. Since $\mathcal{L} \mathcal{C}(\mathrm{P})=\lim _{\longleftarrow} \mathcal{L} \mathcal{C}(\mathrm{P})^{\geq k}$ we are done.

For $i \leq 0$ formula (3.1) is clear. Let us assume $i>0$. Let $d^{\bullet}$ be the differential in $\mathcal{X}^{\bullet}$, and $f: \mathrm{P}(\mu)\langle-i\rangle[i] \rightarrow \mathcal{X}^{-i+1}$ be a non-zero map such that $d^{-i+1} \circ f=0$. Let $\mathcal{Y}^{\bullet}=$ Cone $(f)$ be the cone of $f$. Let $\mathrm{V}$ denote the kernel of $d^{-i+1}$, restricted to $\operatorname{Lev}_{i}\left(\mathcal{X}^{-i+1}\right)$ and $v \in \mathrm{V}$. Since $\operatorname{Lev}_{i}\left(\mathcal{H}^{-i+1} \mathcal{Q}_{\lambda}^{\bullet}\right)=0$, there exists $w \in \operatorname{Lev}_{i}\left(\mathcal{Q}_{\lambda}^{-i}\right)$ such that $d^{-i}(w)=v$. However, $\operatorname{Lev}_{i}\left(\mathcal{Q}_{\lambda}^{-i}\right)=\operatorname{Lev}_{i}\left(\mathcal{X}^{-i}\right)$ by construction, which implies that there exists an indecomposable direct summand, say $\mathrm{M}$, of $\mathcal{X}^{-i}$, such that $d^{-i}(\mathrm{M}) \cong f(\mathrm{P}(\mu)\langle-i\rangle[i])$. It follows that $\mathrm{M} \cong \mathrm{P}(\mu)\langle-i\rangle[i]$, and one can find generators, $a \in \mathrm{M}, b \in \mathrm{P}(\mu)\langle-i\rangle[i]$, such that $d^{-i}(a)=f(b)$. The element $a-b$ thus generates a $\mathbf{C}$-submodule in $\mathcal{Y}^{-i}$, isomorphic to $\mathrm{P}(\mu)\langle-i\rangle[i]$. The latter belongs to the socle of the complex $\mathcal{Y}^{\bullet} \in \mathcal{L} \mathcal{C}(\mathrm{P})$. Hence $\mathcal{Y}^{\bullet}$ splits. This proves (3.1) for $i>0$. Hence, $\mathcal{I}_{\dot{\lambda}}^{\bullet}$ exists and has the required form. The remaining statements then follow by applying Proposition 7(iii).

\section{Quadratic DUALITy FOR POSITIVELy GRADED CATEGORIES}

In this section we develop the abstract theory of quadratic duality in terms of linear complexes. This approach has its origins in $\mathrm{MVS}$ and $\mathrm{MO}$.

Recall that a positively graded category $\mathbf{C}$ is said to be generated in degree one if any morphism in $\mathbf{C}$ is a linear combination of either scalars or compositions of homogeneous morphisms of degree one. Further, $\mathbf{C}$ is called quadratic if it is generated in degree one and any relation for morphisms in $\mathbf{C}$ follows from relations in degree two. The purpose of this section is to describe locally finite-dimensional modules over the quadratic dual category in terms of linear complexes of projectives in the original category. We start by defining the quadratic dual. 
4.1. The quadratic dual of a positively graded category via linear complexes of projectives. Let $\mathbf{C}$ still be a positively graded $\mathbb{k}$-linear category. Let $\mathbf{C}_{0}$ be the subcategory of $\mathbf{C}$ with the same set of objects but only homogeneous morphisms of degree 0 . Then $\mathbf{C}_{1}\left({ }_{-},{ }_{-}\right)$becomes a $\mathbf{C}_{0}$-bimodule in the natural way, which also induces a $\mathbf{C}_{0}$-bimodule structure on $\mathbf{V}=\mathbf{d}\left(\mathbf{C}_{1}\left({ }_{-},{ }_{-}\right)\right)$. Therefore, one can define the free tensor bimodule

$$
\mathbf{C}_{0}[\mathbf{V}]\left({ }_{-},{ }_{-}\right)=\mathbf{C}_{0}\left({ }_{-},{ }_{-}\right) \oplus \mathbf{V}\left({ }_{-},{ }_{-}\right) \oplus\left(\mathbf{V}\left({ }_{-},,_{-}\right) \otimes_{\mathbf{C}_{0}} \mathbf{V}\left({ }_{-},,_{-}\right)\right) \oplus \ldots
$$

and the corresponding category $\mathbf{F}$, where $\mathrm{Ob}(\mathbf{F})=\mathrm{Ob}(\mathbf{C})$, and for $\lambda, \mu \in \mathrm{Ob}(\mathbf{F})$ we have $\mathbf{F}(\lambda, \mu)=\mathbf{C}_{0}[\mathbf{V}](\lambda, \mu)$.

For $\lambda, \mu, \nu \in \mathrm{Ob}(\mathbf{C})$ consider the multiplication map

$$
\mathbf{m}_{\lambda, \mu}^{\nu}: \quad \mathbf{C}_{1}(\nu, \mu) \otimes \mathbf{C}_{1}(\lambda, \nu) \longrightarrow \mathbf{C}_{2}(\lambda, \mu),
$$

which gives rise to the dual map

$$
\mathbf{d}\left(\mathbf{m}_{\lambda, \mu}^{\nu}\right): \mathbf{d}\left(\mathbf{C}_{2}(\lambda, \mu)\right) \rightarrow \mathbf{d}\left(\mathbf{C}_{1}(\nu, \mu) \otimes \mathbf{C}_{1}(\lambda, \nu)\right) \cong \mathbf{d}\left(\mathbf{C}_{1}(\lambda, \nu)\right) \otimes \mathbf{d}\left(\mathbf{C}_{1}(\nu, \mu)\right)
$$

Note that the canonical isomorphism as indicated in (4.1) exists by property (C-iii) and $\left[\mathrm{McL}\right.$, Page 147]. We denote by $\mathbf{J}\left({ }_{-},,_{-}\right)$the subbimodule of $\mathbf{C}_{0}[\mathbf{V}]\left({ }_{-},{ }_{-}\right)$, generated by the images of all these maps, and define the (positively) graded category $\mathbf{C} !$, called the quadratic dual of $\mathbf{C}$, as follows: We just have $\mathrm{Ob}\left(\mathbf{C}^{!}\right)=\mathrm{Ob}(\mathbf{C})=\mathrm{Ob}(\mathbf{F})$, and for $\lambda, \mu \in \mathrm{Ob}\left(\mathbf{C}^{!}\right)$we set

$$
\mathbf{C}^{!}(\lambda, \mu)=\mathbf{F}(\lambda, \mu) / \mathbf{J}(\lambda, \mu)
$$

By definition, the quadratic dual is quadratic.

The following statement was originally proved in [MVS, Theorem 2.4] for unital algebras; an alternative proof was given in [MO, Theorem 8]. The latter one can be adjusted to the setup of the graded categories:

Theorem 12. There is an equivalence of categories,

$$
\epsilon=\epsilon_{\mathbf{C}}: \quad \mathcal{L} \mathcal{C}(\mathrm{P}) \cong \mathbf{C}^{!} \text {-gfmod, }
$$

such that $\epsilon\langle i\rangle[-i] \cong\langle-i\rangle \epsilon$.

Proof. We will use the identification (2.2) and define an equivalence $\epsilon^{\prime}: \mathcal{L} \mathcal{C}(\mathrm{P}) \cong$ $\left(\mathbf{C}^{!}\right)^{\mathbb{Z}}$-fmod. We start by defining the inverse functor. Let $X$ be an object from $\left(\mathbf{C}^{!}\right)^{\mathbb{Z}}$-fmod. In particular, for any $(\lambda, i) \in \mathrm{Ob}\left(\mathbf{C}^{\mathbb{Z}}\right)$ we have $\operatorname{dim} X(\lambda, i)<\infty$. For $i \in \mathbb{Z}$ let $\left(\mathrm{M}_{X}\right)^{i}$ be the graded $\mathbf{C}$-module

$$
\left(\mathrm{M}_{X}\right)^{i}=\bigoplus_{\lambda \in \mathrm{Ob}(\mathbf{C})} \mathrm{P}(\lambda)\langle i\rangle \otimes X(\lambda, i)
$$

(this means we just take $\operatorname{dim} X(\lambda, i)$ many copies of $\mathrm{P}(\lambda)\langle i\rangle)$. We consider the graded $\mathbf{C}$-module $\left(\mathbf{M}_{X}\right)^{i}$ as a $\mathbf{C}^{\mathbb{Z}}$-module via

$$
(\mathrm{P}(\lambda)\langle i\rangle \otimes X(\lambda, i))(\nu, k)=\mathbf{C}^{\mathbb{Z}}((\lambda, i),(\nu, k)) \otimes X(\lambda, i)
$$

for any $(\nu, k) \in \mathrm{Ob}\left(\mathbf{C}^{\mathbb{Z}}\right)$. We want to construct an object $\mathrm{M}_{X}$ in $\mathcal{L} \mathcal{C}(\mathrm{P})$ with $i$ component $\left(\mathrm{M}_{X}\right)^{i}$. Any object $X$ in $\left(\mathbf{C}^{!}\right)^{\mathbb{Z}}$-fmod is uniquely defined by the following 
data describing the module structure:

(D1) a collection of finite-dimensional vector spaces $X(\lambda, j)$ for any $\lambda \in \operatorname{Ob}(\mathbf{C})$, $j \in \mathbb{Z}$; and

(D2) certain elements

$$
f_{\lambda, \mu, j}^{\prime} \in \mathbb{k}-\mathrm{fmod}\left(\left(\mathbf{C}^{!}\right)^{\mathbb{Z}}((\lambda, j),(\mu, j+1)), \mathbb{k}-\mathrm{fmod}(X(\lambda, j), X(\mu, j+1))\right)
$$

for any $\lambda, \mu \in \mathrm{Ob}(\mathbf{C})$ and $j \in \mathbb{Z}$.

Note that it is enough to consider just the action of morphisms of degree one, since $\mathbf{C}_{0}[\mathbf{V}]\left({ }_{-},{ }_{-}\right)$is generated in degrees zero and one. By the definition of the quadratic dual we have fixed isomorphisms

$$
\left(\mathbf{C}^{!}\right)^{\mathbb{Z}}((\lambda, i),(\mu, i+1)) \cong \mathbf{d}(\mathbf{C}((\mu, i+1),(\lambda, i))) .
$$

We get natural isomorphisms as follows:

$$
\begin{aligned}
& \mathbb{k}-\operatorname{fmod}\left(\left(\mathbf{C}^{!}\right)^{\mathbb{Z}}((\lambda, i),(\mu, i+1)), \mathbb{k}-\operatorname{fmod}(X(\lambda, i), X(\mu, i+1))\right) \\
\cong & \mathbb{k}-\operatorname{fmod}\left(X(\lambda, i) \otimes\left(\mathbf{C}^{!}\right)^{\mathbb{Z}}((\lambda, i),(\mu, i+1)), X(\mu, i+1)\right) \\
\cong & \mathbb{k}-\operatorname{fmod}\left(X(\lambda, i), \mathbf{C}^{\mathbb{Z}}((\mu, i+1),(\lambda, i)) \otimes X(\mu, i+1)\right) .
\end{aligned}
$$

We denote by $f_{\lambda, \mu, i} \in \mathbb{k}$-fmod $\left(X(\lambda, i), \mathbf{C}^{\mathbb{Z}}((\mu, i+1),(\lambda, i)) \otimes X(\mu, i+1)\right)$ the image of $f_{\lambda, \mu, i}^{\prime}$ under (4.4). Hence, $X$ comes along with this collection $f_{\lambda, \mu, i}$ of maps and is uniquely determined by this collection. For any $(\nu, k) \in \mathrm{Ob}\left(\mathbf{C}^{\mathbb{Z}}\right)$ the map $f_{\lambda, \mu, i}$ induces a $\mathbb{k}$-linear map

$$
\begin{aligned}
\mathbf{C}^{\mathbb{Z}}((\lambda, i),(\nu, k)) \otimes X(\lambda, i) & \longrightarrow \mathbf{C}^{\mathbb{Z}}((\mu, i+1),(\nu, k)) \otimes X(\mu, i+1) \\
c \otimes x & \longmapsto(c \otimes \mathrm{id})\left(f_{\lambda, \mu, i}(x)\right) .
\end{aligned}
$$

This construction is obviously natural in $(\nu, k)$. Together with formula (4.3) we therefore get a natural transformation of functors (that is, a morphism of $\mathbf{C}$ modules):

$$
\mathrm{d}_{\lambda, \mu}^{i}: \quad \mathrm{P}(\lambda)\langle i\rangle \otimes X(\lambda, i) \quad \longrightarrow \mathrm{P}(\mu)\langle i+1\rangle \otimes X(\mu, i+1) .
$$

Taking the direct sum defines a morphism of graded $\mathbf{C}$-modules $\mathrm{d}^{i}:\left(\mathrm{M}_{X}\right)^{i} \rightarrow$ $\left(\mathrm{M}_{X}\right)^{i+1}$.

We claim that we in fact constructed a complex. For this we have to consider the compositions $\mathrm{d}_{\mu, \sigma}^{i+1} \circ \mathrm{d}_{\lambda, \mu}^{i}$ for any $\lambda, \mu, \sigma \in \mathrm{Ob}(\mathbf{C})$ and $i \in \mathbb{Z}$. We have to show that the composition

$$
\begin{array}{rll}
X(\lambda, i) & \stackrel{f_{\lambda, \mu, i}}{\longrightarrow} & \mathbf{C}_{1}(\mu, \lambda) \otimes X(\mu, i+1) \\
& \stackrel{\text { id } \otimes f_{\mu, \sigma, i+1}}{\longrightarrow} & \mathbf{C}_{1}(\mu, \lambda) \otimes \mathbf{C}_{1}(\sigma, \mu) \otimes X(\sigma, i+2) \\
& \stackrel{\operatorname{m} \otimes \text { id }}{\longrightarrow} & \mathbf{C}_{2}(\sigma, \lambda) \otimes X(\sigma, i+2)
\end{array}
$$


is zero. Via the isomorphisms (4.4) it is enough to show that the following composition:

$$
\begin{aligned}
& \mathbf{d}\left(\mathbf{C}_{2}(\sigma, \lambda)\right) \quad \stackrel{\mathbf{d}\left(\mathbf{m}_{\sigma, \lambda}^{\mu}\right)}{\longrightarrow} \quad \mathbf{d}\left(\mathbf{C}_{1}(\mu, \lambda) \otimes \mathbf{C}_{1}(\sigma, \mu)\right) \cong \mathbf{C}_{1}^{!}(\mu, \sigma) \otimes \mathbf{C}_{1}^{!}(\lambda, \mu) \\
& \stackrel{f_{\mu, \sigma, j}^{\prime} \otimes f_{\lambda, \mu, j}^{\prime}}{\longrightarrow} \mathbb{k}-\operatorname{fmod}(X(\lambda, j), X(\mu, j+1)) \\
& \otimes \mathbb{k}-\operatorname{fmod}(X(\mu, j+1), X(\sigma, j+2)) \\
& \stackrel{\text { composition }}{\longrightarrow} \mathbb{k}-\operatorname{fmod}(X(\lambda, j), X(\sigma, j+2))
\end{aligned}
$$

is zero. The latter is obviously satisfied by the definition of the quadratic dual category $\mathbf{C}^{!}$and the fact that $X$ is a $\left(\mathbf{C}^{!}\right)^{\mathbb{Z}}$-module. Altogether, we defined a functor $\eta:\left(\mathbf{C}^{!}\right)^{\mathbb{Z}}-$ fmod $\rightarrow \mathcal{L} \mathcal{C}(\mathrm{P})$.

Let $\mathcal{P}_{\lambda}^{\bullet}$ be the projective cover of $\mathrm{P}(\lambda)^{\bullet}$ in $\mathcal{L} \mathcal{C}(\mathrm{P})$ (see Proposition 11). We define a functor

$$
\epsilon^{\prime}: \quad \mathcal{L} \mathcal{C}(\mathrm{P}) \longrightarrow\left(\mathbf{C}^{!}\right)^{\mathbb{Z}} \text {-fmod }
$$

as follows: If $\mathcal{M}^{\bullet}$ is an object from $\mathcal{L} \mathcal{C}(\mathrm{P})$, then we define

$$
\epsilon^{\prime}\left(\mathcal{M}^{\bullet}\right)(\lambda, i)=\mathcal{L} \mathcal{C}\left(\mathcal{P}_{\lambda}^{\bullet}\langle-i\rangle[i], \mathcal{M}^{\bullet}\right) .
$$

From the definitions it follows that $\operatorname{dim} \mathcal{L} \mathcal{C}\left(\mathcal{P}_{\lambda}^{\bullet}\langle-i\rangle[i], \mathcal{M}^{\bullet}\right)$ is the multiplicity of $\mathrm{P}(\lambda)^{\bullet}\langle-i\rangle[i]$ in $\mathcal{M}^{\bullet}$. This number is finite by the definition of $\mathcal{L} \mathcal{C}(\mathrm{P})$. Since $\mathcal{M}^{\bullet}$ is a complex of graded $\mathbf{C}$-modules, its differential $d^{j}$ induces a map

$$
\Psi: \mathbf{C}\left((\lambda, i),_{-}\right) \otimes \epsilon^{\prime}\left(\mathcal{M}^{\bullet}\right)(\lambda, i) \rightarrow \mathbf{C}\left((\mu, i+1),_{-}\right) \otimes \epsilon^{\prime}\left(\mathcal{M}^{\bullet}\right)(\mu, i+1)
$$

for any $\lambda, \mu, i$; in particular,

$$
\Psi_{(\lambda, i)}: \mathbf{C}((\lambda, i),(\lambda, i)) \otimes \epsilon^{\prime}\left(\mathcal{M}^{\bullet}\right)(\lambda, i) \rightarrow \mathbf{C}((\mu, i+1),(\lambda, i)) \otimes \epsilon^{\prime}\left(\mathcal{M}^{\bullet}\right)(\mu, i+1) .
$$

Since $\Psi_{(\lambda, i)}$ is a morphism of $\mathbf{C}$-modules, it is uniquely determined by the induced $\mathbb{k}$-linear map

$$
\epsilon^{\prime}\left(\mathcal{M}^{\bullet}\right)(\lambda, i) \rightarrow \mathbf{C}((\mu, i+1),(\lambda, i)) \otimes \epsilon^{\prime}\left(\mathcal{M}^{\bullet}\right)(\mu, i+1) .
$$

Using formula (4.4) we get a possible data $(D 2)$ defining a $\left(\mathbf{C}^{!}\right)^{\mathbb{Z}}$-module structure on $\epsilon^{\prime}(M)$. Using again the formulas (4.7) and (4.6) we get that this is in fact a module structure. Hence $\epsilon^{\prime}\left(\mathcal{M}^{\bullet}\right)$ becomes an object in $\left(\mathbf{C}^{!}\right)^{\mathbb{Z}}$-fmod. From the naturality of the construction it follows that this defines a functor $\epsilon^{\prime}: \mathcal{L} \mathcal{C}(\mathrm{P}) \cong$ $\left(\mathbf{C}^{\mathbb{Z}}\right)$ !-fmod. Together with the identification from (2.2) we get an equivalence $\epsilon$ as asserted in the theorem.

By definition we have $\epsilon\langle i\rangle[-i]) \cong\langle-i\rangle \epsilon$. By construction we have $\epsilon \eta(\mathrm{X}) \cong$ $\mathrm{X}$ and $\eta \epsilon\left(\mathcal{M}^{\bullet}\right) \cong \mathcal{M}^{\bullet}$. Hence $\epsilon$ and $\eta$ are dense. Moreover, by construction, they are both faithful, hence automatically full as well. Therefore, $\epsilon$ and $\eta$ are equivalences of categories. (In fact they are mutually inverses. To see this one has to fix a minimal system of representatives for the isomorphism classes of the indecomposable projective $\mathbf{C}$-modules and work only with projectives from this system.) The theorem follows.

For $k \in \mathbb{Z}$ let $\left(\mathbf{C}^{!}\right)^{\geq k}$ denote the full subcategory of $\mathbf{C}^{!}$, whose objects are $(\lambda, i)$, where $\lambda \in \mathrm{Ob}(\mathbf{C})$ and $i \geq k$. The $\left(\mathbf{C}^{!}\right)^{\geq k}$-fmod can be considered as full subcategories of $\left(\mathbf{C}^{!}\right)^{\mathbb{Z}}$-fmod. The inclusions $\left(\mathbf{C}^{!}\right)^{\geq k+1} \hookrightarrow\left(\mathbf{C}^{!}\right)^{\geq k}$ induce an inverse system on $\left(\mathbf{C}^{!}\right)^{\geq k}$-fmod via truncations, and we have $\left(\mathbf{C}^{!}\right)^{\mathbb{Z}}$-fmod $=\lim _{(}\left(\mathbf{C}^{!}\right)^{\geq k}$-fmod $)$. 
Corollary 13. (a) Let $k \in \mathbb{Z}$. The equivalence $\epsilon$ restricts to an equivalence

$$
\epsilon^{\geq k}: \mathcal{L} \mathcal{C}(\mathbf{P})^{\geq k} \cong\left(\mathbf{C}^{!}\right)^{\geq k} \text {-fmod. }
$$

(b) For any $k \in \mathbb{Z}$, the category $\mathcal{L} \mathcal{C}(\mathrm{P})^{\geq k}$ has enough projectives. Moreover, $\mathcal{P}_{\lambda}\langle-i\rangle[i] \in \mathcal{L} \mathcal{C}(\mathrm{P}) \geq k$ for any $i \leq k$ and $\lambda \in \mathrm{Ob}(\mathbf{C})$.

Proof. This follows directly from Theorem 12 and Lemma 6 .

Let $\mathbb{P}_{k}^{\bullet}$ be a fixed minimal projective generator of $\mathcal{L} \mathcal{C}(\mathrm{P}) \geq k$. This is by definition a complex of graded $\mathbf{C}$-modules. If $\mathcal{M}^{\bullet}$ is a complex in $\mathcal{L} \mathcal{C}(\mathrm{P})$, then there is a $\left(\mathbf{C}^{!}\right)^{\geq k}$-module structure on $\mathcal{L} \mathcal{C}(\mathrm{P})\left(\mathbb{P}_{k}^{\bullet}, \mathcal{M}^{\bullet}\right)$ as follows: To each $\lambda \in \mathrm{Ob}(\mathbf{C})$ and $i \geq k$ we assign the space $\mathcal{L} \mathcal{C}(\mathrm{P})\left(\mathcal{P}_{\lambda}^{\bullet}\langle-i\rangle[i], \mathcal{M}^{\bullet}\right)$ (recall that $\mathcal{P}_{\lambda}^{\bullet}\langle-i\rangle[i]$ is the direct summand of $\mathbb{P}_{k}^{\bullet}$, which corresponds to these $\lambda$ and $i$ ).

Proposition 14. The functor $\mathcal{L} \mathcal{C}(\mathrm{P})\left(\mathbb{P}_{k}^{\bullet},{ }_{-}\right)$defines an equivalence of categories $\mathcal{L} \mathcal{C}(\mathrm{P}) \geq k \cong\left(\mathbf{C}^{!}\right)^{\geq k}$-fmod.

Proof. This follows directly form Theorem 12 and Corollary 13 .

If we choose $\mathbb{P}_{k}^{\bullet}$ such that it gives rise to a directed system we directly get the following result

Corollary 15. The functor $\mathcal{C}(\mathbf{C}-$ gMod $)\left(\underline{\lim } \mathbb{P}_{k}^{\bullet},-\right)=\varliminf_{\lim } \mathcal{L} \mathcal{C}(\mathrm{P})\left(\mathbb{P}_{k}^{\bullet},-\right)$ defines an equivalence of categories $\mathcal{L} \mathcal{C}(\mathrm{P}) \cong\left(\mathbf{C}^{!}\right)$-fmod.

4.2. The complex $\mathbb{P}^{\bullet}$. We denote $\mathbb{P}^{\bullet}=\underline{\lim } \mathbb{P}_{k}^{\bullet}$. This should be thought of as playing the role of a minimal projective generator of $\mathcal{L}(\mathrm{P})$; see Corollary 15 , Proposition 11 gives us at least some information about the structure of $\mathbf{C}$-direct summands of $\mathbb{P}^{\bullet}$. We would like to describe the components $\mathbb{P}^{l}$ of $\mathbb{P}$ as well:

Proposition 16. Let $k \in \mathbb{Z}$.

(a) $\mathbb{P}_{k}^{\bullet}$ is a complex of $\mathbf{C}^{\mathbb{Z}}-\left(\mathbf{C}^{!}\right)^{\geq k}$-bimodules, which is projective both as a left and as a right module.

(b) $\mathbb{P}^{\bullet}$ is a complex of $\mathbf{C}^{\mathbb{Z}}-\left(\mathbf{C}^{!}\right)^{\mathbb{Z}}$-bimodules, which is projective both as a left and as a right module.

Proof. Let $\mathbf{C}_{0}$ be the subcategory of $\mathbf{C}$ from Subsection 4.1 and let $l \in \mathbb{Z}$. Then the $\mathbf{C}^{\mathbb{Z}}-\left(\mathbf{C}^{!}\right)^{\geq k}$-bimodule structure on the component $\mathbb{P}_{k}^{l}$ is given by the following: $\mathbb{P}_{k}^{l}((\lambda, i),(\mu, j))=\mathcal{P}_{\mu}^{l}\langle-j\rangle[j](\lambda, i)$ with the obvious assignments on morphisms. We even claim that

$$
\mathbb{P}_{k}^{l} \cong \bigoplus_{\lambda \in \operatorname{Ob}(\mathbf{C})}\left(\mathbf{C}\left((\lambda, l),_{-}\right) \otimes_{\mathbf{C}_{\mathbf{0}}}\left(\mathbf{C}^{!}\right)^{\geq k}\left({ }_{-},(\lambda, l)\right)\right)
$$

if $l \geq k$, which would imply the projectivity. For such $l$ and each $\lambda \in \mathrm{Ob}(\mathbf{C})$ we can choose $0 \neq v_{\lambda} \in \mathbb{P}_{k}^{l}((\lambda, l),(\lambda, l))$ and

$$
0 \neq w_{\lambda} \in(\mathbf{C}((\lambda, l),(\lambda, l))) \otimes_{\mathbf{C}_{\mathbf{o}}}\left(\left(\mathbf{C}^{!}\right)^{\geq k}((\lambda, l),(\lambda, l)) .\right.
$$

Then, sending $w_{\lambda} \mapsto v_{\lambda}$ (for all $\lambda$ ) defines a homomorphism of bimodules, which is surjective. Since the bimodules have the same composition factors, the surjection is an isomorphism. This implies (国), and (b) follows by taking limits. 
4.3. A homological description of the quadratic dual of a category. Given a finite-dimensional Koszul algebra $A$, its Koszul dual is characterized or, depending on the author, even defined, as the Ext-algebra corresponding to the direct sum of all simple modules concentrated in degree zero. In this section we describe an extension of this characterization which applies to our more general setup.

Let $\operatorname{Ext}_{\mathbf{C}}^{\text {lin }}(\mathrm{L})$ denote the full subcategory of $\mathcal{D}(\mathbf{C}$-gfmod), objects of which are all complexes of the form $\mathrm{L}(\lambda)^{\bullet}\langle-i\rangle[i], \lambda \in \mathrm{Ob}(\mathbf{C}), i \in \mathbb{Z}$. Proposition 11 implies a homological characterization of the category $\mathbf{C}^{!}$as follows:

Proposition 17. There is an isomorphism of categories,

$$
\operatorname{Ext}_{\mathbf{C}}^{\operatorname{lin}}(\mathbf{L}) \cong\left(\left(\mathbf{C}^{!}\right)^{\mathbb{Z}}\right)^{\mathrm{op}}
$$

compatible with the natural $\mathbb{Z}$-actions on both sides. In particular, $\operatorname{Ext}_{\mathbf{C}}^{\operatorname{lin}}(\mathrm{L})$ is generated by the elements of degree zero and one.

Proof. For each $\lambda \in \mathrm{Ob}(\mathbf{C})$ and $i \in \mathbb{Z}$ set $\mathcal{Q}_{\lambda, i}=\mathcal{Q}_{\lambda}\langle-i\rangle[i]$. Denote by $\mathcal{A}$ the full subcategory of $\mathcal{D}$ (C-gfmod), whose objects are all complexes $\mathcal{Q}_{\lambda, i}^{\bullet}$, where $\lambda \in$ $\mathrm{Ob}(\mathbf{C})$ and $i \in \mathbb{Z}$. There is an obvious functor

$$
\begin{aligned}
\alpha: \operatorname{Ext}_{\mathbf{C}}^{\operatorname{lin}}(\mathrm{L}) & \longrightarrow \mathcal{A}, \\
\mathrm{L}(\lambda)\langle-i\rangle[i] & \longmapsto \mathcal{Q}_{\lambda, i}^{\bullet},
\end{aligned}
$$

identifying an object with its projective resolution. On the other hand, by Lemma 9 , we get the functor

$$
\beta:=\mathrm{S}_{-1} \mathrm{Q}_{0}: \quad \mathcal{A} \longrightarrow \mathcal{L} \mathcal{C}(\mathrm{P}) .
$$

By Proposition 11 we have $\beta \alpha(\mathrm{L}(\lambda)\langle-i\rangle[i]) \cong \mathcal{I}_{\dot{\lambda}}$. By Theorem 12, to prove the statement of the proposition, it is therefore enough to show that functor $\beta \alpha$ is full and faithful. From the definition of $\alpha$ and $\mathcal{A}$ it is in fact enough to show that $\beta$ is fully faithful. Let $\lambda, \mu \in \mathrm{Ob}(\mathbf{C})$ and $i, j \in \mathbb{Z}$. Since $\mathbf{C}$ is positively graded, it is easy to see that we have

$$
\mathcal{D}(\mathbf{C} \text {-gfmod })\left(\mathrm{Q}_{-1} \mathcal{Q}_{\lambda, i}^{\bullet}, \mathrm{L}(\mu)\langle-j\rangle[j]\right)=0 .
$$

Hence we get the following chain of isomorphisms:

$$
\begin{aligned}
& \mathcal{L} \mathcal{C}(\mathrm{P})\left(\beta \mathcal{Q}_{\lambda, i}^{\bullet}, \beta \mathcal{Q}_{\mu, j}^{\bullet}\right) \\
& \begin{array}{c}
= \\
\text { (Lemma 10] }
\end{array} \\
& \mathcal{L} \mathcal{E}(\mathrm{P})\left(\mathrm{S}_{-1} \mathrm{Q}_{0} \mathcal{Q}_{\lambda, i}^{\bullet}, \mathrm{S}_{-1} \mathrm{Q}_{0} \mathcal{Q}_{\mu, j}^{\bullet}\right) \\
& \cong \quad \mathcal{K}_{\mathbf{C}}^{\vee}\left(\operatorname{incl~S}_{-1} \mathrm{Q}_{0} \mathcal{Q}_{\lambda, i}^{\bullet}, \mathcal{Q}_{\mu, j}^{\bullet}\right) \\
& \cong \quad \mathcal{D}(\mathbf{C} \text {-gfmod })\left(\operatorname{incl~S}_{-1} \mathrm{Q}_{0} \mathcal{Q}_{\lambda, i}, \mathrm{~L}(\mu)\langle-j\rangle[j]\right) \\
& \text { (Proposition } \cong 11 \text { and } \cong \text { (4.8) } \\
& \mathcal{D}\left(\mathbf{C} \text {-gfmod) }\left(\mathcal{Q}_{\lambda, i}^{\bullet}, \mathrm{L}(\mu)\langle-j\rangle[j]\right)\right. \\
& \cong \quad \mathcal{D}(\mathbf{C} \text {-gfmod })(\mathrm{L}(\lambda)\langle-i\rangle[i], \mathrm{L}(\mu)\langle-j\rangle[j]) \\
& \cong \quad \mathcal{A}(\mathrm{L}(\lambda)\langle-i\rangle[i], \mathrm{L}(\mu)\langle-j\rangle[j]) \text {. }
\end{aligned}
$$

Hence, the functor $\beta=\mathrm{S}_{-1} \mathrm{Q}_{0}$ is fully faithful. The claim follows.

Corollary 18. The morphism space of the positively graded category $\operatorname{Ext}_{\mathbf{C}}^{\operatorname{lin}}(\mathrm{L})$ can be generated by elements of degree 0 or 1 only.

Proof. We have $\operatorname{Ext}_{\mathbf{C}}^{\text {lin }}(\mathrm{L}) \cong\left(\left(\mathbf{C}^{!}\right)^{\mathbb{Z}}\right)^{\text {op }}$ by Proposition 17, and the positively graded category $\left(\left(\mathbf{C}^{!}\right)^{\mathbb{Z}}\right)^{\text {op }}$ is quadratic by definition, hence its morphism space is generated in degrees 0 and 1. 
As an immediate consequence of Proposition 17 we also obtain the following statement, which is obvious for Koszul algebras:

Corollary 19. If $A$ is a positively graded algebra of finite homological dimension, then $A^{!}$is finite-dimensional.

Proof. By Proposition 17, $A^{!}$is a subalgebra of the ext-algebra $\operatorname{Ext}_{A}^{*}(\mathrm{~L}, \mathrm{~L})$, which is finite-dimensional, because $A$ is assumed to have finite homological dimension.

\section{ThE QUADRATIC DUALITY FUNCTOR}

The purpose of this section is to introduce what we call the quadratic duality functor. In some sense it is a generalization of the Koszul duality functor for positively graded Koszul algebras. We will start by stating some general abstract nonsense. For details we refer for example to [Ke1] and De].

Let $\mathcal{A}$ and $\mathcal{B}$ be two arbitrary $\mathbb{k}$-linear categories and $\mathcal{X}^{\bullet}$ be a complex of $\mathcal{A}$ - $\mathcal{B}$ bimodules. Then we have the inner Hom functor

$$
\operatorname{Hom}_{\mathcal{A}}^{\bullet}\left(\mathcal{X}^{\bullet},{ }_{-}\right): \mathcal{C}(\mathcal{A}) \rightarrow \mathcal{C}(\mathcal{B})
$$

as defined in [GM, III.6.14]. At the same time, for any complex $\mathcal{Z}^{\bullet}$ of $\mathcal{B}$-modules we have the associated bicomplex $\mathcal{X}^{\bullet} \otimes_{\mathcal{B}} \mathcal{Z}^{\bullet}$. Applying the functor Tot of taking the total complex defines a functor,

$$
\mathcal{X}^{\bullet} \otimes_{\mathcal{B}-}: \mathcal{C}(\mathcal{B}) \rightarrow \mathcal{C}(\mathcal{A}) .
$$

These functors form an adjoint pair $\left(\mathcal{X}^{\bullet} \otimes_{\mathcal{B}}, \operatorname{Hom}_{\mathcal{A}}^{\bullet}\left(\mathcal{X}^{\bullet},{ }_{-}\right)\right)$.

5.1. Definition and the main theorem. Recall from Proposition 16 that $\mathbb{P}^{\bullet}$ is a complex of $\mathbf{C}_{-}^{\mathbb{Z}} \mathbf{C}^{!^{\mathbb{Z}}}$-bimodules. Hence by the general definition above we have the following pair of functors:

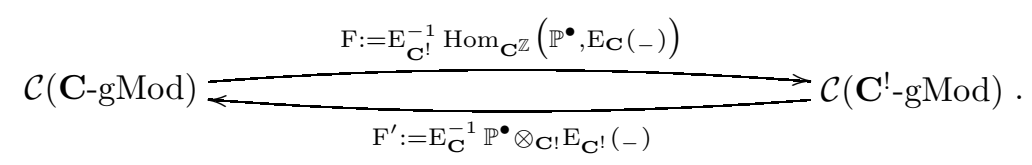

Proposition 20. Let $\mathbf{C}$ be a positively graded category.

(i) The functors $\mathrm{F}$ and $\mathrm{F}^{\prime}$ as in (5.1) form a pair $\left(\mathrm{F}^{\prime}, \mathrm{F}\right)$ of adjoint functors.

(ii) For every $\mathcal{X}^{\bullet} \in \mathcal{C}(\mathbf{C}$-gfmod $), \mathcal{Y}^{\bullet} \in \mathcal{C}\left(\mathbf{C}^{!}\right.$-gfmod $), i, j \in \mathbb{Z}$, we have

$$
\begin{aligned}
& \mathrm{F}(\mathcal{X} \bullet j\rangle[i])=(\mathrm{F} \mathcal{X} \bullet)\langle-j\rangle[i+j], \\
& \mathrm{F}^{\prime}\left(\mathcal{Y}^{\bullet}\langle j\rangle[i]\right)=\left(\mathrm{F}^{\prime} \mathcal{Y} \bullet\right)\langle-j\rangle[i+j]
\end{aligned}
$$

(iii) For $\lambda \in \mathrm{Ob}(\mathbf{C})$ and $i, j \in \mathbb{Z}$ we have

$$
\begin{aligned}
& \mathrm{F}\left(\mathrm{L}_{\mathbf{C}}(\lambda) \bullet\langle j\rangle[i]\right) \cong \mathrm{I}_{\mathbf{C}^{!}}(\lambda) \bullet\langle-j\rangle[i+j], \\
& \mathrm{F}^{\prime}\left(\mathrm{L}_{\mathbf{C}^{!}}(\lambda)^{\bullet}\langle j\rangle[i]\right) \cong \mathrm{P}_{\mathbf{C}}(\lambda)^{\bullet}\langle-j\rangle[i+j] .
\end{aligned}
$$

(iv) We have

$$
\begin{array}{lll}
\mathrm{F}\left(\mathcal{C}^{\downarrow}(\mathbf{C} \text {-gMod })\right) & \subset & \mathcal{C}^{\uparrow}\left(\mathbf{C}^{!} \text {-gMod }\right), \\
\mathrm{F}^{\prime}\left(\mathcal{C}^{\uparrow}\left(\mathbf{C}^{!} \text {-gMod }\right)\right) & \subset & \mathcal{C}^{\downarrow}(\mathbf{C} \text {-gMod }) .
\end{array}
$$

(v) $\mathrm{F}$ sends acyclic complexes from $\mathcal{C}^{\downarrow}\left(\mathbf{C}\right.$-gMod) to acyclic complexes, and $\mathrm{F}^{\prime}$ sends acyclic complexes from $\mathcal{C}^{\uparrow}\left(\mathbf{C}^{!}\right.$-gMod) to acyclic complexes. 
Proof. The statement (ii) follows from [GM, III.6.14]. The properties $\mathrm{F}(\mathcal{X} \bullet[i])=$ $\left(\mathrm{F} \mathcal{X}^{\bullet}\right)[i]$ and $\mathrm{F}^{\prime}\left(\mathcal{Y}^{\bullet}[i]\right)=\left(\mathrm{F}^{\prime} \mathcal{Y}^{\bullet}\right)[i]$, as well as $\mathrm{F}\left(\mathcal{X}^{\bullet}\langle j\rangle\right)=\left(\mathrm{K} \mathcal{X}^{\bullet}\right)\langle-j\rangle[j]$ and $\mathrm{F}^{\prime}\left(\mathcal{Y}^{\bullet}\langle j\rangle\right)=\left(\mathrm{F}^{\prime} \mathcal{Y}^{\bullet}\right)\langle-j\rangle[j]$, follow immediately from the definitions of $\mathrm{F}, \mathrm{F}^{\prime}$, and $\mathbb{P}^{\bullet}$. This proves (iii).

From Theorem 12 and Proposition [(1ii) we have

$$
\mathrm{F}^{\prime}\left(\mathrm{L}_{\mathbf{C}^{!}}(\lambda)\right)=\mathrm{P}_{\mathbf{C}}(\lambda)
$$

From the definition of $\mathbb{P}^{\bullet}$ it follows immediately that $M^{\bullet}:=F\left(L_{C}(\lambda)^{\bullet}\right)$ is concentrated in position 0 (i.e. it is in fact a $\mathbf{C}^{!}$-module). We have to show that $\mathbf{M}$ is an indecomposable injective module. To see this we calculate

$$
\begin{aligned}
& \mathbf{C}^{!} \text {-gfmod }\left(\mathrm{L}_{\mathbf{C}^{!}}(\mu)\langle k\rangle, \mathrm{M}^{0}\right) \\
\cong & \mathcal{C}\left(\mathbf{C}^{!} \text {-gfmod }\right)\left(\mathrm{L}_{\mathbf{C}^{!}}(\mu)^{\bullet}\langle k\rangle, \mathrm{M}^{\bullet}\right) \\
\cong & \mathcal{C}\left(\mathbf{C}^{!} \text {-gfmod }\right)\left(\mathrm{L}_{\mathbf{C}^{!}}(\mu)^{\bullet}\langle k\rangle, \mathrm{F}\left(\mathrm{L}_{\mathbf{C}}(\lambda)^{\bullet}\right)\right) \\
\cong & \mathcal{C}\left(\mathbf{C}_{\text {-gfmod }}\right)\left(\mathrm{F}^{\prime}\left(\mathrm{L}_{\mathbf{C}^{!}}(\mu)^{\bullet}\langle k\rangle\right), \mathrm{L}_{\mathbf{C}}(\lambda)^{\bullet}\right) \\
\cong & \mathcal{C}(\mathbf{C} \text {-gfmod })\left(\mathrm{P}_{\mathbf{C}}(\mu)^{\bullet}\langle-k\rangle[k], \mathrm{L}_{\mathbf{C}}(\lambda)^{\bullet}\right) \\
\cong & \begin{cases}\mathbb{k}, & \lambda=\mu \text { and } k=0, \\
0, & \text { otherwise. }\end{cases}
\end{aligned}
$$

Here, the isomorphism (5.4) follows by adjointness, and the isomorphism (5.5) is given by (5.3). This implies that that module $\mathrm{M}^{0}$ has the simple socle $\mathrm{L}_{\mathbf{C}^{!}}(\lambda)$. Now to prove that $\mathbf{M}^{0} \cong \mathrm{I}_{\mathbf{C}^{!}}(\lambda)$ it remains to compare the characters: Let $\mu \in \mathbf{C}$, $i \geq 0$, and $m_{\mu, \lambda}^{i}$ denote the multiplicity of $\mathrm{P}(\lambda)$ as a direct summand of the zero component of the complex $\mathcal{P}_{\mu}^{\bullet}\langle-i\rangle[i]$. From the definition of $\mathrm{F}$ we have that the dimension of $\mathrm{M}^{0}(\mu)_{-i}$ equals $m_{\mu, \lambda}^{i}$, that is, the composition multiplicity of $\mathrm{L}_{\mathbf{C}^{!}}(\lambda)$ in $\mathbf{P}_{\mathbf{C}^{!}}(\mu)\langle i\rangle$. The latter equals the composition multiplicity of $\mathbf{L}_{\mathbf{C}^{!}}(\mu)\langle i\rangle$ in $\mathbf{I}_{\mathbf{C}^{!}}(\lambda)$ (as both numbers equal the dimension of $\left.\operatorname{Hom}_{\mathbf{C}^{!}}\left(\mathbf{P}_{\mathbf{C}^{!}}(\mu)\langle i\rangle, \mathbf{I}_{\mathbf{C}^{!}}(\lambda)\right)\right)$. Now statement (iii) follows from (iii).

Statement (iv) follows from (iii) and (iii) by a direct calculation.

Finally, to prove (业) we first note the following simplification: If $\mathcal{X}^{\bullet} \in$ $\mathcal{C}^{\downarrow}\left(\mathbf{C}\right.$-gMod), $\lambda \in \operatorname{Ob}(\mathbf{C})$, and $i \in \mathbb{Z}$, then from the construction of $\mathcal{P}_{\dot{\lambda}}^{\bullet}$ it follows that the bicomplex of vector spaces $\operatorname{Hom}_{\mathrm{C}^{\mathbb{Z}}}\left(\mathcal{P}_{\lambda}^{\bullet}\langle-i\rangle[i], \mathcal{X}^{\bullet}\right)$ has only finitely many non-zero components, moreover, they all are finite-dimensional. Hence in the definition of the functor $\mathrm{F}$ all direct products which occur are finite direct products of finite-dimensional spaces. Hence they coincide with the corresponding direct sums.

Now let $\mathcal{X}^{\bullet} \in \mathcal{C}^{\downarrow}\left(\mathbf{C}\right.$-gMod) be an acyclic complex of graded $\mathbf{C}^{!}$-modules. Then we can write $\mathcal{X}^{\bullet}$ as a direct sum of acyclic complexes of vector spaces of the form $0 \rightarrow \mathrm{V} \rightarrow \mathrm{W} \rightarrow 0$, where $\mathrm{V} \cong \mathrm{W} \cong \mathbb{k}\langle i\rangle$ is such that both $\mathrm{V}$ and $\mathrm{W}$ are annihilated by all but one $e_{\lambda}$. Denote by $\mathrm{V}^{\prime}$ and $\mathrm{W}^{\prime}$ the subspace of $\mathrm{F}\left(\mathcal{X}^{\bullet}\right)$, which consist of all those homomorphisms in which the images of the generators of indecomposable projective summands of $\mathbb{P}^{\bullet}$ belong to $\mathrm{V}$ and $\mathrm{W}$, respectively. From the definitions it is obvious that both $V^{\prime}$ and $W^{\prime}$ are in fact $\mathbf{C}^{\prime}$-modules. From (iii) it even follows that both $\mathrm{V}^{\prime}$ and $\mathbf{W}^{\prime}$ are indecomposable injective $\mathbf{C}^{\prime}$-modules. The differential in $\mathcal{X}^{\bullet}$ induces an isomorphism $\mathrm{V}^{\prime} \cong \mathrm{W}^{\prime}$ of these $\mathbf{C}^{\prime}$-modules. This means that 
the complex $\mathrm{F}\left(\mathcal{X}^{\bullet}\right)$ decomposes into a direct sum of acyclic complexes of the form $0 \rightarrow \mathrm{V}^{\prime} \cong \mathrm{W}^{\prime} \rightarrow 0$, and hence is acyclic. For the functor $\mathrm{F}^{\prime}$ the proof is similar (and even easier, as we do not have any direct product in the definition at all). This completes the proof.

Thanks to Proposition 20 we can restrict the functors $\mathrm{F}$ and $\mathrm{F}^{\prime}$ to subcategories $\mathcal{C} \downarrow\left(\mathbf{C}\right.$-gMod) and $\mathcal{C}^{\uparrow}\left(\mathbf{C}^{!}\right.$-gMod $)$, respectively, and then derive the picture (5.1) in the following way:

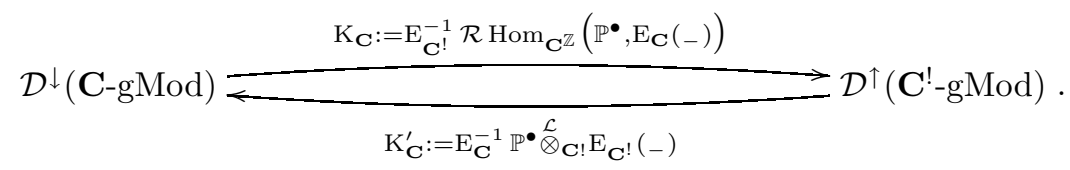

The functors $\mathrm{K}=\mathrm{K}_{\mathbf{C}}$ and $\mathrm{K}^{\prime}=\mathrm{K}_{\mathbf{C}}^{\prime}$ are what we call quadratic duality functors. We emphasize once more that we have

$$
\mathrm{K}=\mathcal{R}\left(\left.\mathrm{F}\right|_{\mathcal{C} \downarrow(\mathbf{C} \text {-gMod })}\right) \quad \text { and } \quad \mathrm{K}^{\prime}=\mathcal{L}\left(\left.\mathrm{F}^{\prime}\right|_{\mathcal{C}^{\uparrow}\left(\mathbf{C}^{!} \text {-gMod }\right)}\right) .
$$

The following alternative description clearly depicts the importance of the equivalence $\epsilon$ from Theorem 12 (namely, $\mathrm{K}^{\prime}$ is just the equivalence $\epsilon^{-1}$ extended to the derived category followed by taking the total complex):

Proposition 21. Up to an isomorphism of functors, the following diagram commutes:

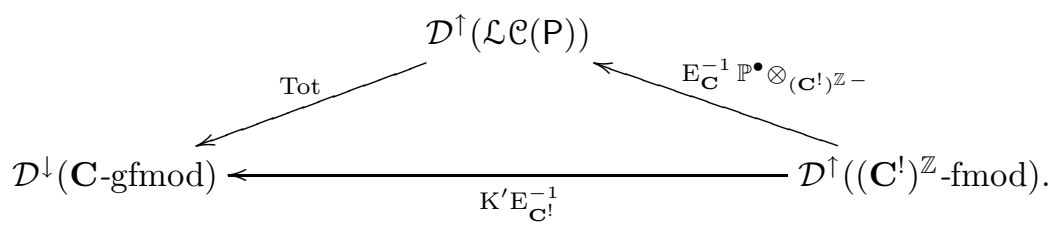

Proof. This follows directly from the definitions and Proposition 16(b).

Our main statement here is the following:

Theorem 22 (Quadratic duality). Let $\mathbf{C}$ be a (positively graded) category.

(i) $\left(\mathrm{K}^{\prime}, \mathrm{K}\right)$ is a pair of adjoint functors.

(ii) For every $\mathcal{X}^{\bullet} \in \mathcal{D}^{\downarrow}\left(\mathbf{C}\right.$-gfmod), $\mathcal{Y}^{\bullet} \in \mathcal{D}^{\uparrow}\left(\mathbf{C}^{!}\right.$-gfmod), $i, j \in \mathbb{Z}$, we have

$$
\begin{aligned}
& \mathrm{K}(\mathcal{X} \bullet\langle j\rangle[i])=\left(\mathrm{K} \mathcal{X}^{\bullet}\right)\langle-j\rangle[i+j] \\
& \mathrm{K}^{\prime}\left(\mathcal{Y}^{\bullet}\langle j\rangle[i]\right)=\left(\mathrm{K}^{\prime} \mathcal{Y}^{\bullet}\right)\langle-j\rangle[i+j]
\end{aligned}
$$

(iii) For $\lambda \in \mathrm{Ob}(\mathbf{C})$ and $i, j \in \mathbb{Z}$ we have

$$
\begin{aligned}
& \mathrm{K}\left(\mathrm{L}_{\mathbf{C}}(\lambda) \bullet\langle j\rangle[i]\right) \cong \mathrm{I}_{\mathbf{C}^{!}}(\lambda)^{\bullet}\langle-j\rangle[i+j] \\
& \mathrm{K}^{\prime}\left(\mathrm{L}_{\mathbf{C}^{!}}(\lambda)^{\bullet}\langle j\rangle[i]\right) \cong \mathrm{P}_{\mathbf{C}}(\lambda)^{\bullet}\langle-j\rangle[i+j] .
\end{aligned}
$$

Proof. The statement (ii) follows from general nonsense (see e.g. [Ke1, 8.1.4] or De]). The rest follows follows from the definitions and Proposition 20. 
5.2. The duality functors applied to modules. Calling $\mathrm{K}$ a duality functor might be too optimistic, in particular, since $\mathrm{K}$ is not an equivalence in general (see e.g. [Ke1, Proposition 8.1.4] and also Theorem [30). However, later on we will see many "duality-like" effects in our situation, which, from our point of view, justify this usage. Our first general observation is the following:

Proposition 23. (i) Let $\mathbf{X} \in \mathbf{C}$-gfmod $\cap \mathcal{D}^{\downarrow}(\mathbf{C}$-gfmod $)$. Then

$$
\mathrm{KX} \bullet \in \mathcal{L} \mathcal{C}\left(\mathrm{I}_{\mathbf{C}^{!}}\right) \cap \mathcal{D}^{\uparrow}\left(\mathbf{C}^{!} \text {-gfmod }\right) \text {. }
$$

(ii) The functor Tot from Proposition 21 sends non-zero objects to non-zero objects.

Proof. Let $\mathrm{X}=\mathrm{X}_{0} \supset \mathrm{X}_{1} \supset \ldots$ be a decreasing filtration of $\mathrm{X}$ such that for every $i=0,1, \ldots$ the module $\mathbf{X}_{i} / \mathbf{X}_{i+1}$ is semi-simple and concentrated in a single degree, say $k_{i}$. Since $\mathbb{P}^{\bullet}$ is a complex of projective $\mathbf{C}$-modules, analogously to the proof of Theorem 22(iii), the module $\mathbf{X}_{i} / \mathbf{X}_{i+1}$ gives rise to an injective $\mathbf{C}^{!}$-module, which is, however, shifted by $\left\langle-k_{i}\right\rangle\left[k_{i}\right]$ because of Theorem 22(ii). Claim (ii) follows.

Every element from $\mathcal{D}^{\uparrow}(\mathcal{L} \mathcal{C}(\mathrm{P}))$ is a double complex of projective $\mathbf{C}$-modules, linear in one direction, and isomorphic to a direct sum of trivial complexes and complexes of the form

$$
\cdots \rightarrow 0 \rightarrow \mathrm{M} \rightarrow 0 \rightarrow \ldots
$$

in the other direction. Moreover, it is acyclic in $\mathcal{D}^{\uparrow}(\mathcal{L} \mathcal{C}(\mathrm{P}))$ if and only if no direct summands of the form (5.8) in the second direction occur. Since the image of Tot is a complex of a projective $\mathbf{C}$-module, bounded from the right, we obtain that the image is acyclic if and only if the bicomplex we started with was acyclic. The claim (iii) follows, and the proof of Proposition 23 is complete.

In case $\mathbf{C}$ is a quadratic category, the functors $\mathrm{K}$ and $\mathrm{K}^{\prime}$ are particularly wellbehaved as we will now illustrate. We first show that the functor $\mathrm{K}^{\prime}$ for $\mathbf{C}$ can be realized using the functor $K$ for $\left(\mathbf{C}^{!}\right)^{\text {op }}$ (which means that these two functors are in fact dual to each other).

Proposition 24. Assume that $\mathbf{C}$ is quadratic. Then

$$
\mathrm{K}_{\left(\mathbf{C}^{!}\right) \text {op }} \cong \mathbb{D} \mathrm{K}_{\mathbf{C}}^{\prime} \mathbb{D} \text {. }
$$

Proof. First let $\mathrm{M} \in \mathbf{C}^{!}$-gfmod be such that $\mathrm{M}_{i}=0$ for all big enough $i$. Further, let $\mathbf{X}=\mathbf{C} \otimes_{\mathbf{C}_{0}} \mathbf{C}^{!}$. Then $\mathbf{X}$ is a graded projective $\mathbf{C}-\mathbf{C}^{!}$-bimodule, which has a unique decomposition into a direct sum of indecomposable projective $\mathbf{C}-\mathbf{C}^{\text {!-bimodules of }}$ the form $\mathbf{N}_{\lambda}=\mathbf{C}\left(\lambda,{ }_{-}\right) \otimes \mathbf{C}_{0} \mathbf{C}^{!}\left({ }_{-}, \lambda\right)$, where $\lambda \in \mathbf{C}$, each occurring with multiplicity one (this multiplicity is given by the dimension of the homomorphism space to the appropriate simple bimodule). Under these assumptions the graded left $\mathbf{C}$ module $\mathbf{X} \otimes_{\mathbf{C}}$ ! $\mathbb{D}$ M has finite-dimensional graded components (and we even have $\left(\mathbf{X} \otimes_{\mathbf{C}^{!}} \mathbb{D M}\right)_{i}=0$ for all small enough $\left.i\right)$. Then the module $\mathbb{D}\left(\mathbf{X} \otimes_{\mathbf{C}^{!}} \mathbb{D} \mathbf{M}\right)$ is a welldefined right $\mathbf{C}$-module with finite-dimensional graded components. Moreover, this module is isomorphic to the module $\operatorname{Hom}_{\mathbb{k}}\left(\mathbf{X} \otimes_{\mathbf{C} !} \mathbb{D M}, \mathbb{k}\right)$ as a graded $\mathbf{C}$-module by definition of $\mathbb{D}$ (note that we understand the Hom-functor using the definitions from the last part of Subsection 2.2). From the definition of $N_{\lambda}$ and Subsection 2.3 it follows that the graded components of the graded vector spaces in the formula 
(5.9) below are finite-dimensional, and hence the adjunction morphism defines an isomorphism of these components:

$$
\Phi: \operatorname{Hom}_{\mathbb{k}}\left(\mathrm{N}_{\lambda} \otimes_{\mathbf{C}^{!}} \mathbb{D} M, \mathbb{k}\right) \cong \operatorname{Hom}_{\mathbf{C}^{!}}\left(\mathbb{D M}, \operatorname{Hom}_{\mathbb{k}}\left(\mathrm{N}_{\lambda}, \mathbb{k}\right)\right) .
$$

The space on the right hand side of (5.9) is nothing other than $\operatorname{Hom}_{\mathbf{C}^{!}}\left(\mathbb{D M}, \mathbb{D N}_{\lambda}\right)$ which is naturally isomorphic to $\operatorname{Hom}_{\left(\mathbf{C}^{\prime}\right)^{\text {op }}}\left(\mathrm{N}_{\lambda}, \mathrm{M}\right)$ by applying the duality $\mathbb{D}$. Now, from the definition of $\mathrm{X}$ and the assumptions on $\mathrm{M}$ it follows that there is a natural isomorphism of graded right $\mathbf{C}$-modules with finite-dimensional graded components:

$$
\Phi: \mathbb{D}\left(\mathbf{X} \otimes_{\mathbf{C}^{!}} \mathbb{D} M\right) \cong \operatorname{Hom}_{\left(\mathbf{C}^{!}\right)^{\text {op }}}(\mathrm{X}, \mathbf{M}) .
$$

Now let $\mathbf{M}^{\bullet} \in \mathcal{C}^{\uparrow}\left(\mathbf{C}^{!}\right.$-gfmod $)$and $\mathbf{X}^{\bullet}=\mathbb{P}^{\bullet}$. Then, by Proposition 16, the components of $\mathbf{X}^{\bullet}$ are (up to shift) isomorphic to the bimodule $\mathbf{X}$ above. Hence it follows that $\Phi$ induces a natural isomorphism

$$
\Phi^{i, j}: \mathbb{D}\left(\mathbf{X}^{i} \otimes_{\mathbf{C}^{!}}(\mathbb{D} \mathbf{M})^{j}\right) \cong \operatorname{Hom}_{\left(\mathbf{C}^{!}\right) \text {op }}\left(\mathbf{X}^{i}, \mathbf{M}^{j}\right)
$$

for any $i, j \in \mathbb{Z}$. The naturality of $\Phi$ induces an isomorphism of bicomplexes

$$
\mathbb{D}\left(\mathbf{X}^{\bullet} \otimes_{\mathbf{C}^{!}} \mathbb{D M}^{\bullet}\right) \cong \operatorname{Hom}_{\left(\mathbf{C}^{!}\right)^{\text {op }}}\left(\mathbf{X}^{\bullet}, \mathrm{M}^{\bullet}\right) .
$$

By the arguments from the proof of Proposition 20, taking the total complex reduces to taking direct sums of finitely many non-zero spaces. Hence the above induces an isomorphism of the corresponding total complexes. The isomorphism $\mathbb{D K}_{\mathbf{C}}^{\prime} \mathbb{D} \cong$ $\mathrm{K}_{\left(\mathbf{C}^{\prime}\right)^{\text {op }}}$ therefore follows from the definition of the involved functors.

For $\lambda \in \mathrm{Ob}(\mathbf{C})$ let $\mathcal{Q}_{\lambda^{\prime}}$ ' denote a minimal projective resolution of $\mathrm{L}_{\left(\mathbf{C}^{!}\right)^{\text {op }}}(\lambda) \in$ $\left(\mathbf{C}^{!}\right)^{\text {op }}$-gfmod. The following result says that the images of indecomposable projective (resp. injective) modules under the functor $\mathrm{K}$ (resp. $\mathrm{K}^{\prime}$ ) is nothing other than the linear part of a minimal injective (projective) resolution of the corresponding simple module.

Proposition 25. Let $\mathbf{C}$ be a positively graded category. Then there are isomorphisms

(i) $\mathrm{K}^{\prime} \mathrm{I}_{\mathbf{C}^{!}}(\lambda) \cong \mathcal{I}_{\dot{\lambda}}^{\bullet} \cong \mathrm{S}_{-1} \mathrm{Q}_{0} \mathcal{Q}_{\lambda}^{\bullet}$ of objects in $\mathcal{L} \mathcal{C}\left(\mathrm{P}_{\mathbf{C}}\right) \cap \mathcal{D}^{\downarrow}(\mathbf{C}$-gfmod $)$, and

(ii) $\mathrm{KP}_{\mathbf{C}}(\lambda) \bullet \cong \mathbb{D} \mathrm{S}_{-1}^{\left(\mathbf{C}^{!}\right)^{\text {op }}} \mathrm{Q}_{0}^{\left(\mathbf{C}^{!}\right)^{\text {op }}} \mathcal{Q}_{\lambda^{\prime}}$ ! of objects in $\mathcal{L} \mathcal{C}\left(\mathbf{I}_{\mathbf{C}^{!}}\right) \cap \mathcal{D}^{\uparrow}\left(\mathbf{C}^{!}\right.$-gfmod $)$in case $\mathrm{C}$ is quadratic.

Proof. Let $\lambda \in \mathrm{Ob}(\mathbf{C})$. From Proposition 21, Theorem 12 and Proposition 11(b) we know that $\mathrm{K}^{\prime} \mathrm{I}_{\mathrm{C}^{!}}(\lambda) \cong \mathcal{I}_{\lambda}^{\bullet} \cong \mathrm{S}_{-1} \mathrm{Q}_{0} \mathcal{Q}_{\lambda}^{\bullet}$. This proves (ii). From this (iii) follows using Proposition 24 .

For quadratic $\mathbf{C}$ we have $\left(\left(\mathbf{C}^{!}\right)^{\text {op }}\right)^{!}=\left(\left(\mathbf{C}^{!}\right)^{!}\right)^{\text {op }}=\mathbf{C}^{\text {op }}$ canonically and from Theorem 12 and Proposition 7 it follows immediately that the categories $\mathbf{C}$-gfmod $\cap$ $\mathcal{D}^{\downarrow}(\mathbf{C}$-gfmod $)$ and $\mathcal{L} \mathcal{C}\left(\mathrm{I}_{\mathbf{C}^{!}}\right) \cap \mathcal{D}^{\uparrow}\left(\mathbf{C}^{\prime}\right.$-gfmod $)$ are equivalent. This equivalence can also be realized in the following way:

Proposition 26. Assume that $\mathbf{C}$ is quadratic. Then

$$
\mathrm{K}: \mathbf{C} \text {-gfmod } \cap \mathcal{D}^{\downarrow}(\mathbf{C} \text {-gfmod }) \longrightarrow \mathcal{L} \mathcal{C}\left(\mathrm{I}_{\mathbf{C} !}\right) \cap \mathcal{D}^{\uparrow}\left(\mathbf{C}^{!} \text {-gfmod }\right)
$$

is an equivalence. 
Proof. From the arguments in the proof of Proposition 23 it follows immediately that the functor

$$
\mathrm{K}: \mathbf{C} \text {-gfmod } \cap \mathcal{D}^{\downarrow}(\mathbf{C} \text {-gfmod }) \rightarrow \mathcal{L} \mathcal{C}\left(\mathrm{I}_{\mathbf{C}^{!}}\right) \cap \mathcal{D}^{\uparrow}\left(\mathbf{C}^{!} \text {-gfmod }\right)
$$

is exact. By Proposition 25((ii) and Proposition 11(b), $\mathrm{K}$ sends indecomposable projective objects from $\mathbf{C}$-gfmod to the corresponding indecomposable projective objects from $\mathcal{L} \mathcal{C}\left(\mathrm{I}_{\mathbf{C}^{!}}\right)$. By $[\mathrm{MO}$, Lemma 6$]$, the induced map on the morphisms is an isomorphism when restricted to the part of degree 1. Hence it is an isomorphism, since $\mathbf{C}$ is quadratic. This completes the proof.

5.3. Quadratic dual functors. Let $\mathbf{C}$ be a positively graded category and $\Lambda \subset$ $\mathrm{Ob}(\mathbf{C}), \Lambda \neq \varnothing$. We denote by $\mathbf{C}_{\Lambda}$ the full subcategory of $\mathbf{C}$ such that $\mathrm{Ob}\left(\mathbf{C}_{\Lambda}\right)=\Lambda$. The category $\mathbf{C}_{\Lambda}$ obviously inherits a positive grading. Let $\mathbf{B}_{\Lambda}$ be the $\mathbf{C}^{\mathbb{Z}}-\left(\mathbf{C}_{\Lambda}\right)^{\mathbb{Z}}$ bimodule $\mathbf{C}\left({ }_{-},{ }_{-}\right)$, which means that it maps the object $((\lambda, i),(\mu, j))$ to $\mathbf{C}(\lambda, \mu)_{j-i}$, where $\mu \in \mathrm{Ob}(\mathbf{C}), \lambda \in \mathrm{Ob}\left(\mathbf{C}_{\Lambda}\right)$. Further, we define the category ${ }_{\Lambda} \mathbf{C}$ as follows: $\operatorname{Ob}\left({ }_{\Lambda} \mathbf{C}\right)=\Lambda$, and for $\lambda, \mu \in \mathrm{Ob}\left({ }_{\Lambda} \mathbf{C}\right)$, the space ${ }_{\Lambda} \mathbf{C}(\lambda, \mu)$ is the quotient of $\mathbf{C}(\lambda, \mu)$ modulo the subspace, generated by all morphisms, which factor through some object outside $\Lambda$. Let ${ }_{\Lambda} \mathbf{D}$ be the $\left.\left(\mathbf{C}^{!}\right)^{\mathbb{Z}}-{ }_{\Lambda} \mathbf{C}^{!}\right)^{\mathbb{Z}}$-bimodule such that ${ }_{\Lambda} \mathbf{D}((\mu, i),(\lambda, j))={ }_{\Lambda}\left(\mathbf{C}^{!}\right)(\mu, \lambda)_{j-i}$ if $\mu \in \Lambda$ and which is the trivial vector space otherwise. The assignments for the maps are the obvious ones. The following observation (which was made in $[\mathrm{Ma}, 3.1]$ ) about the connections between $\mathbf{C}_{\Lambda}$ and ${ }_{\Lambda}\left(\mathbf{C}^{!}\right)$is easy but crucial:

Lemma 27. There is an isomorphism of categories

$$
\tau:\left(\mathbf{C}_{\Lambda}\right)^{!} \text {-gfmod } \cong{ }_{\Lambda}\left(\mathbf{C}^{!}\right) \text {-gfmod, }
$$

such that $\tau \mathrm{M}(\lambda)=\mathrm{M}(\lambda)$ for any $\lambda \in \Lambda$ and $\tau \mathrm{M}(f)=\mathrm{M}(f)$ for any morphism $f$ homogeneous of degree one.

Proof. Let $\lambda, \mu \in \Lambda$. The equalities

$$
\begin{aligned}
& \left(\left(\mathbf{C}_{\Lambda}\right)^{!}\right)_{1}(\lambda, \mu)=\mathbf{d}\left(\left(\mathbf{C}_{\Lambda}\right)_{1}(\mu, \lambda)\right)=\mathbf{d}\left(\mathbf{C}_{1}(\mu, \lambda)\right) \\
& \quad=\mathbf{d}\left(\left({ }_{\Lambda} \mathbf{C}\right)_{1}(\mu, \lambda)\right)=\left(\left({ }_{\Lambda} \mathbf{C}\right)^{!}\right)_{1}(\lambda, \mu)
\end{aligned}
$$

give rise to an identification of the morphisms of degree one. It is easy to see that this gives rise to an identification $\left(\left(\mathbf{C}_{\Lambda}\right)^{!}\right)(\lambda, \mu)=\left(\left({ }_{\Lambda} \mathbf{C}\right)^{!}\right)(\lambda, \mu)$, and the statement follows.

Motivated by the Koszul duality (as proved in [RH]) between translation functors and Zuckerman functors for the classical Bernstein-Gelfand-Gelfand category $\mathcal{O}$, we would like to extend the above equivalence to the following correspondence on functors:

Theorem 28. Let $\mathbf{C}$ be a positively graded category and $\Lambda \subset \mathrm{Ob}(\mathbf{C}), \Lambda \neq \varnothing$. Then the following diagrams commute up to an isomorphism of functors:
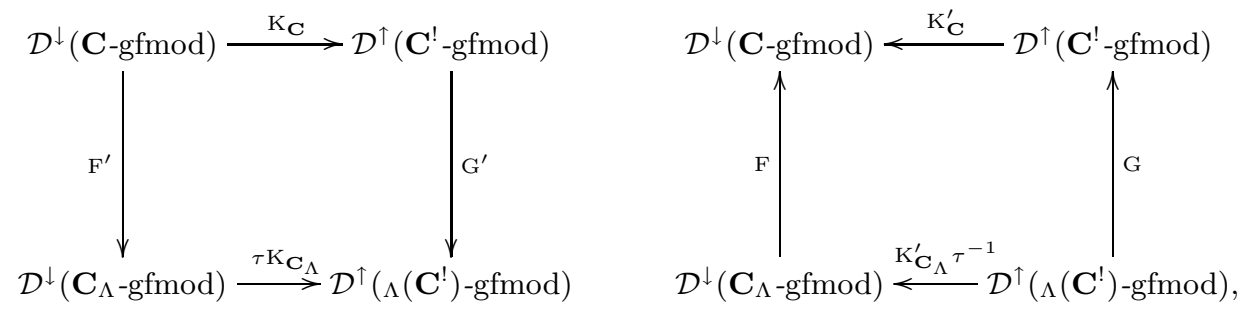
where

$$
\begin{aligned}
& \mathrm{F}=\mathrm{E}_{\mathbf{C}}^{-1} \mathbf{B}_{\Lambda} \stackrel{\mathcal{L}}{\otimes}_{\left(\mathbf{C}_{\Lambda}\right)^{\mathbb{Z}}} \mathrm{E}_{\mathbf{C}_{\Lambda}}(-), \quad \mathrm{G}=\mathrm{E}_{\mathbf{C}^{!} \Lambda}^{-1} \mathbf{D}^{\mathcal{L}}{\left.\stackrel{\mathcal{Q}}{(\Lambda}\left(\mathbf{C}^{!}\right)\right)^{\mathbb{Z}}}_{\mathrm{E}_{\Lambda}\left(\mathbf{C}^{!}\right)}(-),
\end{aligned}
$$

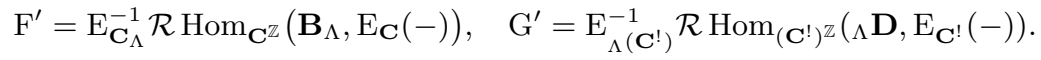

Proof. Since the diagrams are adjoint to each other, it is enough to prove the commutativity of the second, say. We have the natural restriction functor res : $\mathbf{C}$-gfmod $\rightarrow \mathbf{C}_{\Lambda}$-gfmod which is isomorphic to $\mathbf{C}$-gfmod $\left(\mathbf{B}_{\Lambda,-}\right)$ and has the right adjoint ind given by tensoring with $\mathbf{B}_{\Lambda}$. On the other hand we have the natural functor $\mathrm{J}:{ }_{\Lambda}\left(\mathbf{C}^{!}\right)$-gfmod $\rightarrow \mathbf{C}^{!}$-gfmod, which is given by $\mathrm{J}(\mathrm{M})(\lambda)=\mathrm{M}(\lambda)$ if $\lambda \in \Lambda$ and $\mathrm{J}(\mathrm{M})(\lambda)=\{0\}$ otherwise, and on morphisms $\mathrm{J}(\mathrm{M})(f)=\mathrm{M}(f)$ if $\mathrm{M}(f)$ is defined and $\mathrm{J}(\mathrm{M})(f)=0$ otherwise. In other words: $\mathrm{J} \cong{ }_{\Lambda} \mathbf{D} \otimes_{\Lambda}\left(\mathbf{C}^{!}\right)^{\mathbb{Z}}-$. We claim that there is an isomorphism of functors as follows:

$$
\epsilon_{\mathbf{C}}^{-1} \mathrm{~J} \cong \operatorname{ind} \epsilon_{\mathbf{C}_{\Lambda}}^{-1} \tau^{-1} \text {. }
$$

This can be checked by an easy direct calculation. The commutativity of the second diagram above then follows directly from Theorem 12 and Proposition 21.

Remark 29. The statement of Theorem 28 resembles the equivalence of categories, given by Auslander's approximation functor from [Au, Section 5]. A substantial part of the "easy direct calculation" in the proof repeats the calculation, used to establish the fact that Auslander's functor is an equivalence of certain categories.

5.4. The Koszul duality theorem. We call a positively graded category $\mathbf{C}$ Koszul provided that the minimal projective resolution of $\mathrm{L}(\lambda) \in \mathbf{C}$-gmod is linear for every $\lambda$. This generalizes the usual definition of Koszul algebras (see e.g. [BGS, Section 2]). It is of course not a big surprise that for Koszul categories all of our previous results can be seriously strengthened. Our main result here is the following:

Theorem 30 (Koszul duality). Let $\mathbf{C}$ be a positively graded category. The following conditions are equivalent:

(a) $\mathbf{C}$ is Koszul.

(b)

$$
\mathcal{D}^{\downarrow}(\mathbf{C} \text {-gfmod }) \underset{\mathrm{K}_{\mathbf{C}}^{\prime} !}{\rightleftarrows} \mathcal{D}^{\uparrow}\left(\mathbf{C}^{!} \text {-gfmod }\right)
$$

are mutually inverse equivalences of categories.

(c) $\operatorname{KP}_{\mathbf{C}}(\lambda)^{\bullet} \cong \mathrm{L}_{\mathbf{C}^{!}}(\lambda)^{\bullet}$ for every $\lambda \in \mathrm{Ob}(\mathbf{C})$.

(d) $\mathrm{K}^{\prime} \mathrm{I}_{\mathbf{C}^{!}}(\lambda)^{\bullet} \cong \mathrm{L}_{\mathbf{C}}(\lambda)^{\bullet}$ for every $\lambda \in \mathrm{Ob}(\mathbf{C})$.

(e) The functor Tot from (21) is dense.

Proof. (国) $\Rightarrow($ b $)$. We assume that $\mathbf{C}$ is Koszul. Since we have an adjoint pair of functors $\left(\mathrm{K}_{\mathbf{C}^{\prime}}^{\prime}, \mathrm{K}_{\mathbf{C}}\right.$ ) (see Theorem $[22$ ), it is enough to show that the adjunction morphisms are isomorphisms. We even claim that it is enough to show that the adjunction morphisms are isomorphisms for any simple object. Indeed, by the definition of $\mathcal{D}^{\downarrow}\left(\mathbf{C}\right.$-gfmod), for any $\mathcal{X}^{\bullet} \in \mathcal{D}^{\downarrow}(\mathbf{C}$-gfmod $), \lambda \in \mathrm{Ob}(\mathbf{C})$ and $i \in \mathbb{Z}$, the bicomplex

$$
\operatorname{Hom}_{\mathbf{C}-\operatorname{gfmod}}\left(\mathcal{P}_{\lambda}^{\bullet}\langle-i\rangle[i], \mathcal{X}^{\bullet}\right)
$$

has only finitely many non-zero components, each of which is a finite-dimensional vector space. Hence the claim that the adjunction morphism is an isomorphism for 
$\mathcal{X}$ follows from the corresponding statement for simple objects by taking the limit as in Corollary 13

Now let us prove the statement for simple objects. We can of course assume that these simple objects are concentrated in position zero. From Proposition 25 we have $\mathrm{KL}_{\mathbf{C}}(\lambda)=\mathrm{I}_{\mathbf{C}^{!}}(\lambda)^{\bullet}$, and we also have an isomorphism $\mathrm{K}^{\prime} \mathrm{I}_{\mathbf{C}^{!}}(\lambda) \cong \mathrm{S}_{-1} \mathrm{Q}_{0} \mathcal{Q}_{\lambda}^{\bullet}$. The latter one is isomorphic to $\mathcal{Q}_{\lambda}^{\bullet}$, since $\mathbf{C}$ is Koszul. Hence $\mathrm{K}^{\prime} \mathrm{K} \mathrm{L}_{\mathbf{C}}(\lambda) \cong \mathrm{L}_{\mathbf{C}}(\lambda)$. Since the adjunction morphism $\mathrm{K}^{\prime} \mathrm{KL}_{\mathbf{C}}(\lambda) \rightarrow \mathrm{L}_{\mathbf{C}}(\lambda)$ is non-zero, it must be an isomorphism. Via the duality $\mathbb{D}$ we could also say that $\mathbf{C}$ is Koszul provided that the minimal injective resolution of $\mathrm{L}(\lambda) \in \mathbf{C}$-gmod is linear for every $\lambda$. Note that $\mathbf{C}$ is quadratic by BGS, Corollary 2.3.3]. Again using Theorem 22)(iii) and Proposition 25] we get, completely analogous to our previous argument, that the adjunction morphism ID $\rightarrow \mathrm{KK}^{\prime}$ is an isomorphism. This implies (b).

(b) $\Rightarrow$ (回). By Proposition 25 we have $\mathrm{KL}_{\mathbf{C}}(\lambda)^{\bullet} \cong \mathrm{I}_{\mathbf{C}^{!}}(\lambda)^{\bullet}$ for any $\lambda \in \mathrm{Ob}(\mathbf{C})$. From Theorem 22(1ii) and (iii) we know that $\mathrm{K}^{\prime} \mathrm{KL}_{\mathbf{C}}(\lambda)^{\bullet}$ is a linear complex of projective $\mathbf{C}$-modules. Since, by assumption, $\mathrm{K}^{\prime} \mathrm{KL}_{\mathbf{C}}(\lambda)^{\bullet} \cong \mathrm{L}_{\mathbf{C}}(\lambda)^{\bullet}$, the module $\mathrm{L}_{\mathbf{C}}(\lambda)^{\bullet}$ has a linear projective resolution, which implies (国).

(国) $\Leftrightarrow$ (d). From Proposition 25 we get $\mathrm{K}^{\prime} \mathrm{I}_{\mathrm{C}^{!}}(\lambda) \cong \mathrm{S}_{-1} \mathrm{Q}_{0} \mathcal{Q}_{\lambda}^{\bullet}$, and the statement is clear.

(回) $\Rightarrow$ (IC). This follows from Propositions 11 and 25] since any Koszul category is quadratic ([BGS, Corollary 2.3.3]).

(ㄷ) $\Rightarrow$ (a) . Since we assume $\operatorname{KP}(\lambda)^{\bullet}=\mathrm{L}_{\mathbf{C}^{!}}(\lambda)^{\bullet}$ for every $\lambda \in \mathrm{Ob}(\mathbf{C})$, Proposition 23 implies that the minimal injective resolution of any $L_{\mathbf{C}^{!}}(\lambda)^{\bullet}$ is linear, hence $\left(\mathbf{C}^{!}\right)$is Koszul, and therefore so is $\left(\mathbf{C}^{!}\right)^{!}$. So, it is enough to show that $\mathbf{C}$ is quadratic. If it is not, then there is some $\lambda \in \mathrm{Ob}(\mathbf{C})$ such that the characters of $\mathrm{P}_{\mathbf{C}}(\lambda)$ and $\mathbf{P}_{\left(\mathbf{C}^{!}\right) !}(\lambda)$ do not agree, since $\mathbf{C}$ is then a proper quotient of $\left(\mathbf{C}^{!}\right)^{!}$(it does have more relations). From Theorem 22(iii) we get that if $\operatorname{KP}_{\mathbf{C}}(\lambda)^{\bullet} \cong \mathrm{L}_{\mathbf{C}^{!}}(\lambda)$, then $\mathrm{KP}_{\left(\mathbf{C}^{!}\right) !}(\lambda) \bullet \mathrm{L}_{\left(\mathbf{C}^{!}\right) ! !}(\lambda) \cong \mathrm{L}_{\mathbf{C}^{!}}(\lambda)$. This however contradicts Theorem 222(iii).

(a) $\Rightarrow$ (国). This follows directly from Proposition 25.

(还) $\Rightarrow$ (国). We only have to show that, if $\mathcal{Q}_{\lambda}^{\bullet}$ is not linear, then the isomorphism class of the minimal projective resolution $\mathcal{Q}_{\lambda}^{\bullet}$ of $\mathrm{L}(\lambda)$ in $\mathcal{D}^{\downarrow}(\mathbf{C}$-gfmod) does not intersect the image of Tot. Assuming the contrary we have $\mathrm{K}^{\prime} \mathcal{X}^{\bullet} \cong \mathcal{Q}_{\lambda}^{\bullet}$ for some $\mathcal{X}^{\bullet} \in \mathcal{D}^{\uparrow}\left(\mathbf{C}^{!}\right.$-gfmod) by Proposition 21, Then $\mathrm{KK}^{\prime} \mathcal{X} \bullet \cong \mathrm{I}_{\mathbf{C}^{!}}(\lambda)$ by Theorem 22(iii) and

$$
\mathcal{Y}^{\bullet}:=\mathrm{K}^{\prime} \mathrm{KK}^{\prime} \mathcal{X}^{\bullet} \cong \mathrm{S}_{1} \mathrm{Q}_{0} \mathcal{Q}_{\lambda}^{\bullet}
$$

by Proposition 11(b). The adjunction of $\mathrm{K}^{\prime}$ and $\mathrm{K}$ (Theorem 22(1i)) implies the existence of maps

$$
\mathcal{Q}_{\lambda}^{\bullet} \rightarrow \mathcal{Y}^{\bullet} \rightarrow \mathcal{Q}_{\lambda}^{\bullet}
$$

whose composition is the identity map. Since both $\mathcal{Q}_{\lambda}^{\bullet}$ and $\mathcal{Y}^{\bullet}$ are complexes of projective modules bounded from the right, the maps in (5.10) can already be realized in the homotopy category (see e.g. [Ha, Chapter III(2), Lemma 2.1]). We obtain that $\mathcal{Q}_{\lambda}^{\bullet}$ is a direct summand of $\mathcal{Y}^{\bullet}$, which is impossible since $\mathcal{Y}^{\bullet}$ is linear and $\mathcal{Q}_{\lambda}^{\bullet}$ is not. The theorem follows.

Remark 31. Analogously to [BGS, 2.13], linear complexes can be interpreted as objects of the core of a non-standard $t$-structure on the category $\mathcal{D}^{\downarrow}(\mathbf{C}$-gfmod) (and other derived categories we consider). In the case of Koszul categories, the Koszul duality functors transform the standard $t$-structure on $\mathcal{D}^{\downarrow}$ (C-gfmod) into the non-standard $t$-structure on $\mathcal{D}^{\uparrow}\left(\mathbf{C}^{!}\right.$-gfmod $)$and vice versa. 
We would like to emphasize the following direct consequence:

Corollary 32. All projective resolutions of simple $\mathbf{C}$-modules are linear if and only if they all belong to the image of the functor Tot from Proposition 21,

\section{Koszul dual FunCtors For the CATEGory $\mathcal{O}$}

In this section we apply the results from Section 5 to Koszul algebras associated with the blocks of the classical Bernstein-Gelfand-Gelfand category $\mathcal{O}$ (see [BGG2, [BGS]). We give an alternative proof of the result of Ryom-Hansen ([RH] $)$ on the Koszul duality of translation and Zuckerman functors on $\mathcal{O}$, and prove the Koszul duality of twisting/completion and shuffling/coshuffling functors. In the next section we will describe several applications, in particular, we will give an alternative proof of the categorification results of Sussan ( $[\mathrm{Su}]$ ) by applying Koszul duality to the corresponding categorification result from [St2].

6.1. Category $\mathcal{O}$ : notation and preliminaries. For any (complex) Lie algebra $\mathfrak{g}$ we denote by $\mathcal{U}(\mathfrak{g})$ its universal enveloping algebra. Let $\mathfrak{g}$ be a complex semisimple Lie algebra with a fixed Cartan subalgebra $\mathfrak{h}$ inside a Borel subalgebra $\mathfrak{b}$. Let $\mathcal{O}=\mathcal{O}(\mathfrak{g})$ be the corresponding category $\mathcal{O}$ from [BGG2] given by all finitely generated $\mathcal{U}(\mathfrak{g})$-modules, which are $\mathfrak{h}$-diagonalizable and locally $\mathcal{U}(\mathfrak{b})$-finite. The morphisms are ordinary $\mathcal{U}(\mathfrak{g})$-homomorphisms. The Weyl group $W$ acts naturally on $\mathfrak{h}^{*}$, via $(x, \lambda) \mapsto x(\lambda)$ for any $x \in W$ and $\lambda \in \mathfrak{h}^{*}$. There is also the so-called "dot-action" $x \cdot \lambda=x(\lambda+\rho)-\rho$ with the fixed point $-\rho$, where $\rho$ is the halfsum of positive roots. It is well-known that the category $\mathcal{O}$ has enough projectives and injectives. For $\mu \in \mathfrak{h}^{*}$ let $L(\mu)$ denote the simple module with the highest weight $\mu, P(\mu)$ denote the indecomposable projective cover and $I(\mu)$ denote the indecomposable injective hull of $L(\mu)$ in $\mathcal{O}$.

The action of the center of $\mathcal{U}(\mathfrak{g})$ decomposes the category into blocks, i.e. $\mathcal{O}=$ $\bigoplus \mathcal{O}_{\chi}$, where (due to the Harish-Chandra isomorphism) the blocks are indexed by the $W$-orbits under the dot-action. We also write $\mathcal{O}_{\chi}=\mathcal{O}_{\lambda}$, if $\lambda \in \chi$ is maximal (in the usual ordering on weights). In particular, $\mathcal{O}_{0}$ denotes the principal block containing the trivial representation, and $P(\mu)$ (resp. $L(\mu)$ or $I(\mu)$ ) is an object of $\mathcal{O}_{\lambda}$ if and only if $\mu \in W \cdot \lambda$. The module $P_{\chi}=\bigoplus_{\mu \in \chi} P(\mu)$ is a minimal projective generator for $\mathcal{O}_{\chi}$, hence $\mathcal{O}\left(P_{\chi}, \bullet\right)$ defines an equivalence of categories between $\mathcal{O}_{\chi}$ and the category of finitely generated (which means finite-dimensional) right $\operatorname{End}_{\mathcal{O}}\left(P_{\chi}\right)$ modules (Bass, Section 2]). From BGS it is known that $A(\chi)=\operatorname{End}_{\mathcal{O}}\left(P_{\chi}\right)$ can be equipped with a positive $\mathbb{Z}$-grading such that the corresponding graded algebra $\mathrm{A}(\chi)$ becomes a Koszul algebra. Since we have always worked with left modules so far, we use the duality on $\mathcal{O}$ to identify $A(\chi) \cong A(\chi)^{o p}$. We denote by $\mathbf{A}(\chi)$ the corresponding positively graded $\mathbb{C}$-category (recall that the objects of $\mathbf{A}(\chi)$ can be considered as a minimal system of representatives of the isomorphism classes of indecomposable projective modules in $A(\chi)$-gfmod with the head concentrated in degree zero and where the morphisms are the morphisms of graded modules; see Subsection 2.1). We will identify the objects of the category $\mathbf{A}(\chi)$ either with isomorphism classes of indecomposable projective objects in $A(\chi)$-fmod, or the isomorphism classes of simple modules in $A(\chi)$-fmod or even just with the corresponding highest weights, depending on what is the most convenient way in any particular situation. 
Then the category $\mathbf{A}(\chi)$-gfmod of all finite-dimensional graded $\mathbf{A}(\chi)$-modules is a "graded version" of $\mathcal{O}_{\chi}$. We will also write $\mathbf{A}(\lambda)$ (resp. $A(\lambda)$ ) instead of $\mathbf{A}(\chi)$ (resp. $A(\chi))$ if $\lambda \in \chi$ is maximal. In particular, we have $\mathbf{A}(0)$-gfmod, the graded version of the principal block $\mathcal{O}_{0}$.

If $P(\mu) \in \mathcal{O}_{\lambda}$, then we have the corresponding indecomposable projective $P(\mu) \in$ $A(\lambda)$-fmod and $\mathbf{P}(\mu)=\mathbf{A}(\lambda)\left(\mu,,_{-}\right) \in \mathbf{A}(\lambda)$-gfmod. Similarly, $L(\mu) \in \mathcal{O}_{\lambda}$ corresponds to a simple module $L(\mu) \in A(\lambda)$-fmod and to $\mathrm{L}(\mu) \in \mathbf{A}(\lambda)$-gfmod, the simple quotient of $\mathrm{P}(\mu)$. Recall that we denoted the injective hull of $\mathrm{L}(\mu)$ by $\mathrm{I}(\mu)$. The indecomposable projective modules in $\mathbf{A}(\lambda)$-gfmod are exactly the modules of the form $\mathrm{P}(\mu)\langle j\rangle$ for some $\mu \in W \cdot \lambda$ and $j \in \mathbb{Z}$.

For more details concerning this graded version of category $\mathcal{O}$ (in the language of modules over graded algebras) we refer to [BGS] and also [St1].

6.2. The parabolic categories ${ }_{\Lambda} A(\chi)$-gfmod. If $\mathfrak{p} \supseteq \mathfrak{b}$ is a parabolic subalgebra of $\mathfrak{g}$, then we denote by $W_{\mathfrak{p}} \subseteq W$ the corresponding parabolic subgroup, and let $\mathcal{O}^{\mathfrak{p}}$ denote the full subcategory of $\mathcal{O}$ given by all locally $\mathcal{U}(\mathfrak{p})$-finite objects. For a $W$-orbit $\chi$ with maximal weight $\lambda$ let $\mathcal{O}_{\chi}^{\mathfrak{p}}=\mathcal{O}_{\lambda}^{\mathfrak{p}}$ be the full subcategory having as objects all the objects from $\mathcal{O}_{\chi}$, which are locally $\mathcal{U}(\mathfrak{p})$-finite. We will also call these categories "blocks", although they are not indecomposable and of course not even non-trivial in general. The Zuckerman functors

$$
\mathrm{Z}_{\lambda}^{\mathfrak{p}}: \mathcal{O}_{\lambda} \rightarrow \mathcal{O}_{\lambda}^{\mathfrak{p}}
$$

are defined as the functors of taking the maximal $\mathfrak{p}$-locally finite quotient. These functors are right exact. Let $\mathrm{Z}^{\mathfrak{p}}=\mathrm{Z}_{0}^{\mathfrak{p}}$ and let $\mathrm{i}^{\mathfrak{p}}$ denote its right adjoint, i.e. $\mathrm{i}^{\mathfrak{p}}$ is nothing other than the inclusion functor $\mathcal{O}_{0}^{\mathfrak{p}} \rightarrow \mathcal{O}_{0}$. Note that $\mathrm{Z}^{\mathfrak{p}} P(x \cdot 0) \neq 0$ if and only if $x \in W^{\mathfrak{p}}$, the set of shortest coset representatives of $W_{\mathfrak{p}} \backslash W$. The module $\mathrm{Z}_{\lambda}^{\mathfrak{p}} P_{\chi}$ is a minimal projective generator for $\mathcal{O}_{\lambda}^{\mathfrak{p}}=\mathcal{O}_{\chi}^{\mathfrak{p}}$. Let $A(\chi)^{\mathfrak{p}}$ denote its endomorphism ring, which is the quotient of $A(\chi)$ modulo the homogeneous ideal generated by all idempotents corresponding to the simple modules which are in $\mathcal{O}_{\chi}$ but not in $\mathcal{O}_{\chi}^{\mathfrak{p}}$. In particular, $A(\chi)^{\mathfrak{p}}$ inherits a (positive) grading from $A(\chi)$. We will consider the positively graded category corresponding to $A(\chi)^{\mathfrak{p}}$ via the correspondence (1.1) and denote it by $\mathbf{A}(\chi)^{\mathfrak{p}}$. Using the language from Subsection 5.3 we have

Lemma 33. There is a canonical isomorphism of categories, $\mathbf{A}(\chi)^{\mathfrak{p}} \cong{ }_{\Lambda}(\mathbf{A}(\chi))$, where $\Lambda=\Lambda(\chi, \mathfrak{p})$ is the set of idempotents corresponding to simple modules in $\mathcal{O}_{\chi}$, which are contained in $\mathcal{O}_{\chi}^{\mathfrak{p}}$.

Proof. This follows directly from the definitions.

6.3. The category $\mathbf{A}(0)_{\Lambda(\mathfrak{p})^{\prime}}$-gfmod. Let $W_{\mathfrak{p}}$ be a parabolic subgroup of $W$. Let $W(\mathfrak{p})=\left\{x \in W \mid x^{-1} w_{0} \in W^{\mathfrak{p}}\right\}$, where $w_{0}$ is the longest element in $W$. Let $\Lambda^{\prime}=\Lambda(\mathfrak{p})^{\prime}$ denote the set of weights of the form $x \cdot 0, x \in W(\mathfrak{p})$. We consider $\Lambda(\mathfrak{p})^{\prime}$ as a subset of $\mathrm{Ob}(\mathbf{A}(0))$. Then the following holds

Lemma 34. A complete system of indecomposable projective objects in the category $\mathbf{A}(0)_{\Lambda^{\prime}}$-gfmod is given by restricting the modules $\mathbf{P}(\lambda)\langle j\rangle \in \mathbf{A}(0)$-gfmod, where $\lambda \in \Lambda^{\prime}, j \in \mathbb{Z}$, to objects in $\mathbf{A}(0)_{\Lambda^{\prime}}$-gfmod.

Proof. This follows directly from the definition of $\mathbf{A}(0)_{\Lambda^{\prime}}$. 
6.4. Koszul duality of translation and Zuckerman functors. Let $\mathfrak{p} \supseteq \mathfrak{b}$ be a parabolic subalgebra and let $\lambda=\lambda(\mathfrak{p}) \in \mathfrak{h}^{*}$ be such that its stabilizer under the dotaction is $W_{\mathfrak{p}}$ and it is maximal in its orbit. In [BGS (and [Ba1]), it is proved that $A(\chi)^{\mathfrak{p}}$ is always a Koszul algebra. More precisely ([BGS, Corollary 3.7.3]), there is an isomorphism of graded algebras $\mathrm{A}(0)^{!} \rightarrow \mathrm{A}(0)$, which induces an isomorphism of categories $\mathbf{A}(0)^{!} \rightarrow \mathbf{A}(0)$, such that the object $x \cdot 0$ is mapped to the object $x^{-1} w_{0} \cdot 0$, where $w_{0}$ is the longest element in $W$. More generally (Ba1, Proposition 3.1]), there is an isomorphism of categories $(\mathbf{A}(\lambda(\mathfrak{p})))^{!} \cong \mathbf{A}(0)^{\mathfrak{p}}$ mapping the object $x \cdot \lambda(\mathfrak{p})$ to the object $x^{-1} w_{0} \cdot 0$. For any $\mathfrak{p}$ we fix such an isomorphism and the induced isomorphism of categories $\sigma^{\mathfrak{p}}: \mathcal{D}^{\uparrow}\left(\mathbf{A}(\lambda(\mathfrak{p}))^{!}\right.$-gfmod $) \cong \mathcal{D}^{\uparrow}\left(\mathbf{A}(0)^{\mathfrak{p}}\right.$-gfmod $)$. Set $\sigma=\sigma^{\mathfrak{b}}$. We have the following Koszul duality functors:

$$
\mathcal{D}^{\downarrow}(\mathbf{A}(0) \text {-gfmod }) \underset{\mathrm{K}_{\mathbf{A}(0)}^{\prime}}{\longleftarrow} \mathcal{D}^{\uparrow}\left(\mathbf{A}(0)^{!} \text {-gfmod }\right) \stackrel{\sigma}{\cong} \mathcal{D}^{\uparrow}(\mathbf{A}(0) \text {-gfmod }),
$$

such that $\mathrm{KL}(x \cdot 0) \cong \mathrm{I}\left(x^{-1} w_{0} \cdot 0\right)$. More generally,

$$
\mathcal{D}^{\downarrow}(\mathbf{A}(\lambda(\mathfrak{p})) \text {-gfmod }) \underset{\mathrm{K}_{\mathbf{A}(\lambda(\mathfrak{p}))}}{\stackrel{\mathrm{K}_{\mathbf{A}(\lambda(\mathfrak{p}))}}{\rightleftarrows}} \mathcal{D}^{\uparrow}\left(\mathbf{A}(\lambda(\mathfrak{p}))^{!} \text {-gfmod }\right) \stackrel{\sigma^{\mathfrak{p}}}{\cong} \mathcal{D}^{\uparrow}\left(\mathbf{A}(0)^{\mathfrak{p}} \text {-gfmod }\right),
$$

such that $\mathrm{K}_{\mathbf{A}(\lambda(\mathfrak{p}))} \mathrm{L}(x \cdot \lambda(\mathfrak{p})) \cong I\left(x^{-1} w_{0} \cdot 0\right)$.

As the algebra $A$ is finite-dimensional, we have that the bounded derived category $\mathcal{D}^{b}(\mathbf{A}(0)$-gfdmod $)$ is by definition contained in $\mathcal{D}^{\downarrow}(\mathbf{A}(0)$-gfmod $)$ as well as in $\mathcal{D}^{\uparrow}(\mathbf{A}(0)$-gfmod). Hence it makes sense to restrict the functors to this subcategory. Since the Koszul functor sends simple modules to injective modules (Theorem 221) and the involved algebra has finite global dimension, we obtain functors as follows (see [BGS, Theorem 2.12.6] for details):

$$
\mathcal{D}^{b}(\mathbf{A}(0) \text {-gfdmod }) \underset{\mathrm{K}_{\mathbf{A}(0)}^{\prime}}{\longleftarrow} \mathcal{D}^{b}\left(\mathbf{A}(0)^{!} \text {-gfdmod }\right) \stackrel{\sigma}{\cong} \mathcal{D}^{b}(\mathbf{A}(0) \text {-gfdmod }) .
$$

With the notation from Lemma 33 the Zuckerman functors induce functors

$$
\begin{aligned}
Z^{\mathfrak{p}}: \mathbf{A}(0) \text {-gfdmod } & \rightarrow \mathbf{A}(0)^{\mathfrak{p}} \text {-gfdmod } \cong{ }_{\Lambda} \mathbf{A}(0) \text {-gfdmod } \\
\mathrm{i}^{\mathfrak{p}}:{ }_{\Lambda} \mathbf{A}(0)^{\mathfrak{p}} \text {-gfdmod } \cong \mathbf{A}(0)^{\mathfrak{p}} \text {-gfdmod } & \rightarrow \mathbf{A}(0) \text {-gfdmod }
\end{aligned}
$$

On the other hand, for any block $\mathcal{O}_{\lambda}$, where $\lambda$ is integral, we have the translation functors

$$
\begin{array}{ll}
\theta_{0}^{\lambda}: & \mathcal{O}_{0} \rightarrow \mathcal{O}_{\lambda}, \\
\theta_{\lambda}^{0}: & \mathcal{O}_{\lambda} \rightarrow \mathcal{O}_{0},
\end{array}
$$

given by translation onto and out of the wall (for details see for example Ja1, [GJ]). They induce functors

$$
\begin{array}{ll}
\theta_{0}^{\lambda}: & \mathbf{A}(0) \text {-fdmod } \rightarrow \mathbf{A}(\lambda) \text {-fdmod }, \\
\theta_{\lambda}^{0}: & \mathbf{A}(\lambda) \text {-fdmod } \rightarrow \mathbf{A}(0) \text {-fdmod }
\end{array}
$$

In [St1] it is proved that the latter have graded lifts

$$
\begin{array}{ll}
\tilde{\theta}_{0}^{\lambda}: & \mathbf{A}(0) \text {-gfdmod } \rightarrow \mathbf{A}(\lambda) \text {-gfdmod }, \\
\tilde{\theta}_{\lambda}^{0}: & \mathbf{A}(\lambda) \text {-gfdmod } \rightarrow \mathbf{A}(0) \text {-gfdmod, }
\end{array}
$$


which give rise to the original functors if we forget the grading. We are mostly interested in the case when $\lambda$ is integral and the stabilizer $W_{\lambda}$ of $\lambda$ is generated by a simple reflection $s$, that is, $\lambda$ is "lying on exactly one wall". To avoid even more notation we restrict ourselves from now on to this case. We fix a standard lift $\tilde{\theta}_{0}^{\lambda}$ such that $\theta_{0}^{\lambda}$ maps $\mathrm{P}(0)$ to $\mathrm{P}(\lambda)$. We fix a standard lift $\tilde{\theta}_{\lambda}^{0}$ of $\theta_{\lambda}^{0}$ such that the adjunction morphism ID $\rightarrow \tilde{\theta}_{\lambda}^{0} \tilde{\theta}_{0}^{\lambda}$ is homogeneous of degree 1 . This means $\tilde{\theta}_{\lambda}^{0} \mathrm{P}(\lambda) \cong \mathrm{P}(s \cdot 0)$. For more details we refer to [St1, Section 1, Section 3.2].

As an application of our general setup we get the following result, conjectured in $\overline{B G S}$, and originally proved in $[\mathrm{RH}]$, concerning the restrictions of the Koszul functors as given in (6.1):

Theorem 35. Let $\mathfrak{p} \supseteq \mathfrak{b}$ be a parabolic subalgebra of $\mathfrak{g}$ such that $W_{\mathfrak{p}}=\{1, s\}$ for some simple reflection s. The following diagrams commute up to isomorphisms of functors:

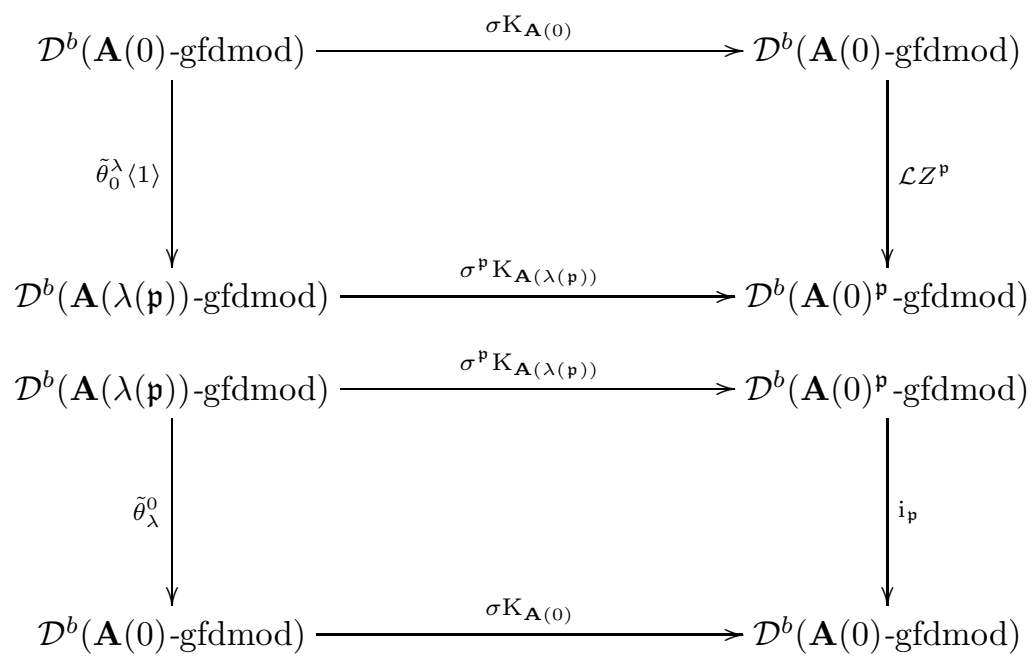

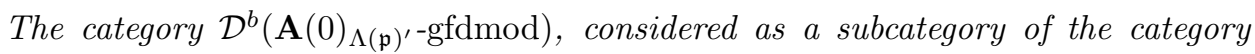
$\mathcal{D}^{b}(\mathbf{A}(0)$-gfdmod $)$, is exactly the image of the translation functor $\tilde{\theta}_{\lambda}^{0}$.

Corollary 36. (i) The functor $\mathrm{i}_{\mathfrak{p}} \mathcal{L} Z^{\mathfrak{p}}\langle 1\rangle[-1]$ and the translation functor $\tilde{\theta}_{s}=$ $\tilde{\theta}_{0}^{\lambda} \tilde{\theta}_{0}^{\lambda}$ through the s-wall are Koszul dual to each other.

(ii) The functor $\mathrm{i}_{\mathfrak{p}} \mathcal{L} Z^{\mathfrak{p}}\langle 1\rangle[-1]$ is both left and right adjoint to itself.

Proof. (ii) follows directly from Theorem 35 and Theorem 22(1ii). The statement

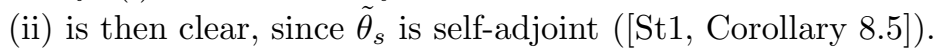

Remark 37. Our result differs from the one in $[\mathrm{RH}]$ by a shift in the grading. This is because in $[\mathrm{RH}]$ the graded lift of the $\theta_{0}^{\lambda}$ is chosen such that it maps a simple module concentrated in degree $k$ to zero or to a simple module concentrated in degree $k$. We chose the lift such that it maps a simple module concentrated in degree $k$ to zero or to a simple module concentrated in degree $k-1$ (see [St1, Theorem 8.1]).

To prove Theorem 35 we use the following auxiliary statement:

Lemma 38. Let $\mathfrak{p} \supseteq \mathfrak{b}$ be a fixed parabolic subalgebra of $\mathfrak{g}$. For any $x \in W(\mathfrak{p})$ there is an isomorphism $\beta_{x}: \mathrm{P}(x \cdot 0) \cong \tilde{\theta}_{\lambda}^{0} \mathrm{P}(x \cdot \lambda(\mathfrak{p})) \in \mathbf{A}(0)$-gfdmod. 
Proof. Using [St1, Theorem 8.4] and [Ja2, 4.12 (3)] we get for any $j \in \mathbb{Z}$ that

$$
\begin{aligned}
& \mathbf{A}(0)-\operatorname{gfdmod}\left(\tilde{\theta}_{\lambda}^{0} P(x \cdot \lambda(\mathfrak{p})), \mathrm{L}(y \cdot 0)\langle j\rangle\right) \\
\cong & \mathbf{A}(0)-\operatorname{gfdmod}\left(\mathrm{P}(x \cdot \lambda(\mathfrak{p})), \tilde{\theta}_{0}^{\lambda} \mathrm{L}(y \cdot 0)\langle j-1\rangle\right)
\end{aligned}
$$

is only non-zero if $y=x$ and $j=0$, in which case it is isomorphic to

$$
\mathbf{A}(0)-\operatorname{gfdmod}(\mathrm{P}(x \cdot \lambda(\mathfrak{p})), \mathrm{L}(x \cdot \lambda(\mathfrak{p})))=\mathbb{C} .
$$

Since the translation functors map projective objects to projective objects, the statement follows.

Proof of Theorem 35. By adjointness it is enough to prove the commutativity of the second diagram. We start with some general statements. Let $x, y \in W(\mathfrak{p})$ and $\mathrm{P}=\mathrm{P}(x \cdot \lambda(\mathfrak{p})), \mathbf{Q}=\mathrm{P}(y \cdot \lambda(\mathfrak{p})) \in \mathbf{A}(\lambda(\mathfrak{p}))$-gfdmod. The functor $\mathrm{T}:=\tilde{\theta}_{\lambda}^{0}$ is exact and does not annihilate any submodule of a given projective module ([Ja2, 4.13 (5) or $\left.\left.\left(3^{\prime}\right)\right]\right)$, hence it induces a natural inclusion

$$
\mathbf{A}(\lambda(\mathfrak{p}))-\operatorname{gfdmod}(\mathrm{P}\langle-1\rangle, \mathrm{Q}) \hookrightarrow \mathbf{A}(0)-\operatorname{gfdmod}\left(\tilde{\theta}_{\lambda}^{0} \mathrm{P}\langle-1\rangle, \tilde{\theta}_{\lambda}^{0} \mathrm{Q}\right)
$$

of graded vector spaces. We claim that this is even an isomorphism. By St1, Theorem 8.4, Proposition 6.7 (2)] we have

$$
\begin{aligned}
& \mathbf{A}(0)-\operatorname{gfdmod}\left(\tilde{\theta}_{\lambda}^{0} \mathrm{P}\langle-1\rangle, \tilde{\theta}_{\lambda}^{0} \mathrm{Q}\right) \\
\cong & \mathbf{A}(0)-\operatorname{gfdmod}\left(\mathrm{P}, \tilde{\theta}_{0}^{\lambda} \tilde{\theta}_{\lambda}^{0} \mathrm{Q}\right) \\
\cong & \mathbf{A}(0)-\operatorname{gfdmod}(\mathrm{P}, \mathrm{Q}\langle 1\rangle \oplus \mathrm{Q}\langle-1\rangle) \\
\cong & \mathbf{A}(0)-\operatorname{gfdmod}(\mathrm{P}\langle-1\rangle, \mathrm{Q}) \oplus \mathbf{A}(0)-\operatorname{gfdmod}(\mathrm{P}\langle 1\rangle, \mathrm{Q}) \\
\cong & \mathbf{A}(0)-\operatorname{gfdmod}(\mathrm{P}\langle-1\rangle, \mathrm{Q}) ;
\end{aligned}
$$

the latter follows from the positivity of the grading. Hence the spaces in (6.4) have the same dimension, and the map has to be an isomorphism. Together with Lemma 34, the functor $\mathrm{T}$ induces an isomorphism

$$
\alpha: \mathbf{A}(\lambda(\mathfrak{p}))_{1} \cong\left(\mathbf{A}(0)_{\Lambda(\mathfrak{p})^{\prime}}\right)_{1} .
$$

From Lemma 38 and Lemma 34 we know that $\mathrm{T}$ induces a functor

$$
\mathrm{T}: \quad \mathcal{L} \mathcal{C}\left(\mathrm{P}_{\mathbf{A}(\lambda(\mathfrak{p}))}\right) \rightarrow \mathcal{L} \mathcal{C}\left(\mathrm{P}_{\mathbf{A}(0)}\right) .
$$

To show that the second diagram in Theorem 35 commutes, it is enough (by Theorem 30 and Proposition 21) to show that the following diagram commutes:

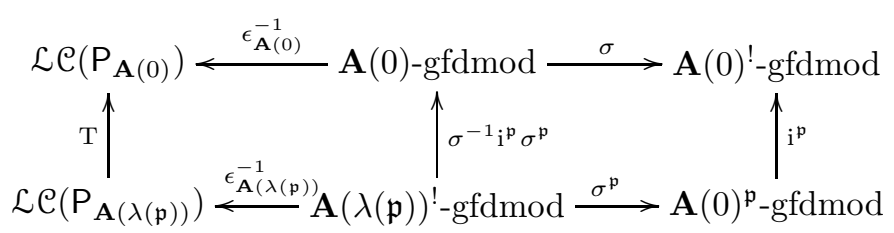

The right hand square commutes by definition. We have the isomorphisms $\beta_{x}$ : $\mathrm{P}(x \cdot 0) \cong \operatorname{TP}(x \cdot \lambda(\mathfrak{p}))$ from Lemma 38. The explicit description of $\epsilon^{-1}$ in the proof of Theorem 12 implies therefore that for any $\mathbf{M} \in \mathbf{A}(\lambda(\mathfrak{p}))^{\text {!-gfdmod, the }}$ components of the complexes $\mathrm{T} \epsilon_{\mathbf{A}(\lambda(\mathfrak{p}))}^{-1}(\mathrm{M})$ and $\epsilon_{\mathbf{A}(0)}^{-1} \sigma^{-1} \dot{i}^{\mathfrak{p}} \sigma^{\mathfrak{p}}(\mathbf{M})$ are isomorphic via the isomorphism $\beta_{x}$. Moreover, the isomorphism $\alpha$ implies that we even have an isomorphism of complexes. This isomorphism is natural by the definition of morphisms in the category of linear complexes of projective modules. Hence the diagram commutes and implies Theorem 35 
6.5. Koszul duality of twisting and shuffling functors. For any simple reflection $s$ let $T_{s}: \mathcal{O}_{0} \rightarrow \mathcal{O}_{0}$ be the twisting functors described for example in [AS]. Let $\mathrm{T}_{s}: \mathbf{A}(0)$-gfdmod $\rightarrow \mathbf{A}(0)$-gfdmod be the graded version of $T_{s}$ such that $\mathrm{T}_{s} \mathrm{P}(0)$ has head $\mathrm{L}(s \cdot 0)$ (FKS, Proposition 5.1]) and let $\mathrm{G}_{s}$ be its right adjoint. This functor is a graded version of Joseph's completion functor ([.], MS1, Theorem 4]). Let $\mathrm{C}_{s}: \mathbf{A}(0)$-gfdmod $\rightarrow \mathbf{A}(0)$-gfdmod denote the graded version of Irving's shuffling functor, which is given by taking the cokernel of the adjunction morphism $\mathrm{ID}\langle-1\rangle \rightarrow \tilde{\theta}_{\lambda}^{0} \tilde{\theta}_{0}^{\lambda}$. Let $\mathrm{D}_{s}$ be its right adjoint, which is given by taking the kernel of the adjunction morphism $\tilde{\theta}_{\lambda}^{0} \tilde{\theta}_{0}^{\lambda} \rightarrow \operatorname{ID}\langle 1\rangle$. In this section we will prove that twisting functors and shuffling functors are Koszul dual to each other:

Theorem 39. For any simple reflection s, the following diagrams commute up to isomorphism of functors:

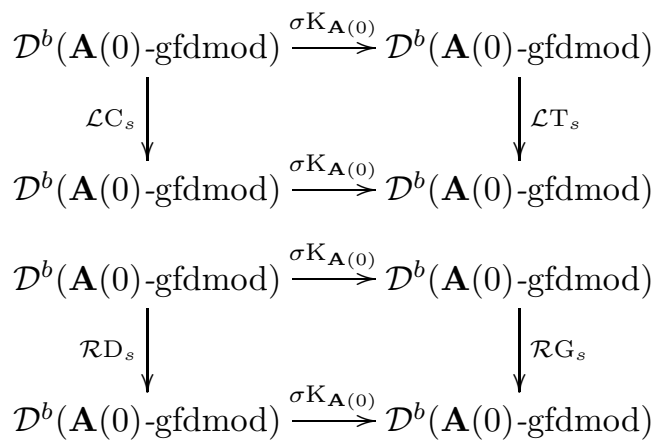

Remark 40. Applying Proposition [24, [MS2, Lemma 5.2] and [KM, Corollary 6], from Theorem 39 it also follows that the following diagrams commute up to an isomorphism of functors:

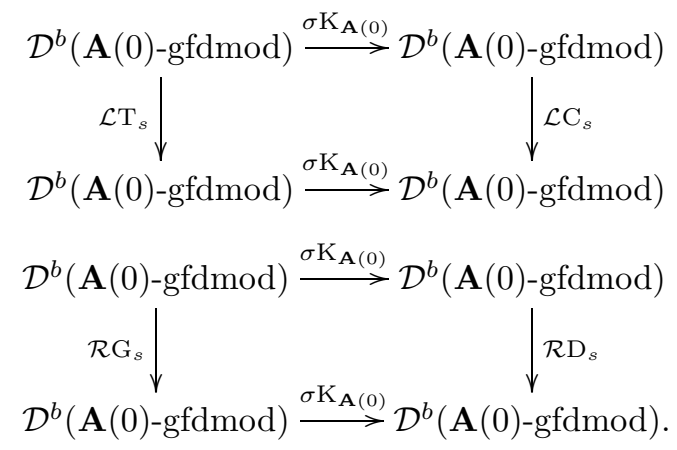

The rest of the section will be devoted to the proof of Theorem 39. We start with the following definition: Let $\mathcal{A}, \mathcal{B}$ be categories and assume $\mathcal{B}$ is abelian. Let

$$
0 \rightarrow \mathrm{F}_{1} \rightarrow \mathrm{F}_{2} \rightarrow \mathrm{F}_{3} \rightarrow 0
$$

be a complex of functors $\mathrm{F}_{i}: \mathcal{A} \rightarrow \mathcal{B}(1 \leq i \leq 3)$. The complex (6.5) is exact if it gives rise to a short exact sequence $0 \rightarrow \mathrm{F}_{1}(M) \rightarrow \mathrm{F}_{2}(M) \rightarrow \mathrm{F}_{3}(M) \rightarrow 0$ in $\mathcal{B}$ for any object $M \in \mathcal{A}$. Analogously, if $\mathcal{B}$ is a triangulated category, then for functors $\mathrm{F}_{i}: \mathcal{A} \rightarrow \mathcal{B}$ we say that $\mathrm{F}_{1} \rightarrow \mathrm{F}_{2} \rightarrow \mathrm{F}_{3} \rightarrow \mathrm{F}_{1}[1]$ is a distinguished triangle if it gives rise to a distinguished triangle in $\mathcal{B}$ when evaluated at any object in $\mathcal{A}$. 
Lemma 41. Let $s$ be a simple reflection and $\mathcal{P} \subseteq \mathbf{A}(0)$-gfdmod be the full additive category given by all projective objects. Then there is an exact sequence of functors from $\mathcal{P}$ to $\mathbf{A}(0)$-gfdmod of the form

$$
0 \rightarrow \mathrm{T}_{s} \rightarrow \mathrm{id}\langle 1\rangle \rightarrow \mathrm{i}_{\mathfrak{p}} \mathrm{Z}^{\mathfrak{p}}\langle 1\rangle \rightarrow 0,
$$

where $\mathfrak{p}$ is the parabolic subalgebra of $\mathfrak{g}$, associated with $s$.

Proof. We have $\mathrm{T}_{s} \mathrm{P}\left(w_{0} \cdot 0\right) \cong \mathrm{P}\left(w_{0} \cdot 0\right)$ by definition. From the proof of MS1, Theorem 6] it follows that $\operatorname{Hom}\left(\mathrm{T}_{s}, \operatorname{ID}\langle 1\rangle\right) \cong C(\mathfrak{h})$, where $C(\mathfrak{h})$ is the coinvariant algebra as in [So, 1.2]. In particular, there is a unique up to scalar natural transformation can of lowest degree. It must be non-trivial on $\mathrm{P}(0)$, otherwise it would be trivial anywhere, since $\mathrm{T}_{s}$ commutes with translation functors through walls (AS, Section 3]). Then the cokernel of can is $i_{\mathfrak{p}} Z^{\mathfrak{p}}\langle 1\rangle$ ([AS, Proposition 5.4]).

Corollary 42. There exists a morphism $\phi$ of functors such that

$$
\mathrm{i}_{\mathfrak{p}} \mathcal{L} \mathrm{Z}^{\mathfrak{p}}\langle 1\rangle[-1] \stackrel{\phi}{\rightarrow} \mathcal{L} \mathrm{T}_{s} \rightarrow \mathrm{id}\langle 1\rangle \rightarrow \mathrm{i}_{\mathfrak{p}} \mathrm{Z}^{\mathfrak{p}}\langle 1\rangle
$$

is a triangle of functors.

Proof. This follows immediately from Lemma 41 (see for example [KS, Proposition 1.8.8]).

Proof of Theorem 39. Since $\mathrm{K}=\mathrm{K}_{\mathbf{A}(0)}$ is a functor of triangulated categories and an equivalence by Theorem 30 , from Corollary 42 we get the triangle

$$
\mathrm{K}^{-1} \mathrm{i}_{\mathfrak{p}} \mathcal{L} \mathrm{Z}^{\mathfrak{p}}\langle 1\rangle[-1] \mathrm{K} \stackrel{\phi}{\rightarrow} \mathrm{K}^{-1} \mathcal{L} \mathrm{T}_{s} \mathrm{~K} \rightarrow \mathrm{K}^{-1} \mathrm{ID}\langle 1\rangle \mathrm{K} \rightarrow \mathrm{K}^{-1} \mathrm{i}_{\mathfrak{p}} \mathrm{Z}^{\mathfrak{p}}\langle 1\rangle \mathrm{K} .
$$

From Theorem 22 we have $\mathrm{K}\langle 1\rangle \cong\langle-1\rangle[1] \mathrm{K}$. Together with Corollary 36 the triangle (6.6) gives rise to a triangle

$$
\tilde{\theta}_{s} \stackrel{\phi^{\prime}}{\rightarrow} \mathrm{K}^{-1} \mathcal{L} \mathrm{T}_{s} \mathrm{~K} \rightarrow \mathrm{ID}\langle-1\rangle[1] \rightarrow \tilde{\theta}_{s}[1]
$$

and therefore to the triangle

$$
\mathrm{K}^{-1} \mathcal{L} \mathrm{T}_{s} \mathrm{~K}[-1] \rightarrow \mathrm{ID}\langle-1\rangle \stackrel{\phi^{\prime \prime}}{\rightarrow} \tilde{\theta}_{s} \rightarrow \mathrm{K}^{-1} \mathcal{L} \mathrm{T}_{s} \mathrm{~K} .
$$

Note that the map $\phi^{\prime \prime}$ is graded (homogeneous of degree zero). Since the graded vector space $\operatorname{Hom}\left(\operatorname{ID}\langle-1\rangle, \tilde{\theta}_{s}\right)$ is one-dimensional in degree zero ([Ba2, Theorem 4.9]), $\phi^{\prime \prime}$ must be the adjunction morphism up to a scalar. Hence we have $\mathrm{K}^{-1} \mathcal{L} \mathrm{T}_{s} \mathrm{~K} \cong$ $\mathcal{L C}_{s}$. Therefore, the first diagram of Theorem 39 commutes. The commutativity of the second follows by adjointness.

\section{Applications}

Finally we would like to indicate applications of our results.

7.1. A categorical version of the quantized Schur-Weyl duality. In FKS a categorification of finite-dimensional quantum $\mathfrak{s l}_{2}$-modules was obtained using certain graded versions of blocks of the category of Harish-Chandra bimodules for $\mathfrak{s l}_{n}$ and translation functors. The quantized Schur-Weyl duality was categorified using certain singular blocks of the category $\mathcal{O}$ together with the action of twisting functors and translation functors through walls (see [FKS, Section 5] based on [BFK, Corollary 1]). The standard and the dual canonical bases were realized using graded versions of Verma modules and simple modules. Now Theorems 35 and 39 provide the Koszul dual version of it: The Schur-Weyl duality can be categorified 
using the bounded derived categories of certain parabolic blocks of $\mathcal{O}$ (as suggested in [BFK, Section 4]) together with the action of shuffling and derived Zuckerman and inclusion functors. From Theorem 22(iii) and [FKS, Theorem 5.3 (e)] it follows directly that the standard and canonical basis can be realized using graded versions of dual Verma modules and injective modules.

7.2. A functorial tangle invariant. J. Sussan proved in $\mathrm{Su}$ that the categorification from [BFK, 3.2.3] of the Temperley-Lieb algebra using singular blocks of category $\mathcal{O}$ together with Zuckerman functors and inclusion functors can be extended to a functorial tangle invariant using derived twisting and derived completion functors. Theorem 39 shows that the functorial invariants of [Su and of [St2] and MS3] are Koszul dual to each other.

7.3. A "Koszul dual" for Harish-Chandra bimodules. The categorification of finite-dimensional quantum $\mathfrak{s l}_{2}$-modules from [BFK] was obtained using certain graded versions of blocks of the category of Harish-Chandra bimodules and translation functors. In general, these graded blocks are not Koszul, hence it does not make sense to speak about a Koszul dual version at all. However, we propose the following alternative to the "Koszul dual" of the graded version of the category ${ }_{\lambda} \mathcal{H}_{\mu}^{1}$ of Harish-Chandra bimodules with generalized central character $\chi_{\lambda}$ from the left hand side and central character $\chi_{\mu}$ from the right hand side: There is the well-known equivalence from [BG] which identifies ${ }_{\lambda} \mathcal{H}_{\mu}^{1}$ with a certain subcategory of $\mathcal{O}_{\lambda}$ (see [BG] or [Ja2, Section 6]). By [Ja2, 6.17] the graded version of ${ }_{\lambda} \mathcal{H}_{\mu}^{1}$ is equivalent to $\mathbf{A}(\lambda)_{\Lambda}$-gfdmod for some $\Lambda$. Hence Lemma 27 provides the quadratic dual, namely ${ }_{\Lambda} \mathbf{A}^{\mathfrak{p}}$-gfdmod, where $\mathbf{A}^{\mathfrak{p}}$-gfdmod is the Koszul dual of $\mathbf{A}(\lambda)$-gfdmod. Using the Koszul duality of translation and Zuckerman functors (Theorem 35 and $[\mathrm{RH}]$ ), we get directly from Theorem 28 a quadratic dual version of the results in FKS.

7.4. A Koszul duality for Kac-Moody Lie algebras. In our opinion, one advantage of our setup using graded categories in comparison with the setup in BGS] is the fact that the categories are allowed to have infinitely many objects. Instead of considering the principal block of the category $\mathcal{O}$ for a semi-simple Lie algebra, we could consider the category $\mathcal{O}$ for a symmetrizable complex Kac-Moody algebra, and in there any regular block outside the critical hyperplanes. Translation functors through walls are defined in Fi1. The generalization of Soergel's structure theorem ([So]) holds (see [Fi2] for the deformed case and [St3] for the non-deformed case). In analogy with [So and St1] the morphism spaces between indecomposable projective objects (if they exist) can be equipped with a positive grading giving rise to a positively graded category $\mathbf{C}$ as defined in Section 2, In case projective objects do not exist, they can be replaced by tilting objects (see [Fi2]). Theorem 22 and Theorem 30 then provide an analogue of the Koszul duality for a regular block outside the critical hyperplanes for the category $\mathcal{O}$ of a symmetrizable complex Kac-Moody algebra.

\section{Appendix A. An abstract generalized Koszul complex}

Some linear algebra. Let $V$ be a finite-dimensional $\mathbb{k}$-vector space and $\mathbf{v}=\left\{v_{i}\right.$ : $i=1, \ldots, n\}$ be a basis in $V$. We denote by $\left\{v^{i}: i=1, \ldots, n\right\}$ the dual basis in 
$V^{*}:=\mathbf{d}(V)$. Then we have a canonical isomorphism,

$$
\begin{aligned}
\varphi: \quad V \otimes_{\mathbb{k}} V^{*} & \stackrel{\sim}{\rightarrow} \operatorname{Hom}_{\mathbb{k}}(V, V), \\
v \otimes w^{*} & \mapsto w^{*}(-) v .
\end{aligned}
$$

We have $I_{V}:=\varphi^{-1}\left(\operatorname{Id}_{V}\right)=\sum_{i=1}^{k} v_{i} \otimes v^{i}$, in particular, the element $I_{V}$ does not depend on the choice of the basis v. Let $H \subset V$ be a subspace and $H^{\perp}=\{f \in$ $V^{*} \mid f(h)=0$ for all $\left.h \in H\right\}$ be the corresponding orthogonal complement in $V^{*}$.

Then we have

$$
\begin{aligned}
& \varphi\left(H \otimes_{\mathbb{k}} V^{*}\right)=\{f: V \rightarrow V \mid \operatorname{Im}(f) \subset H\}, \\
& \varphi\left(V \otimes_{\mathbb{k}} H^{\perp}\right)=\{f: V \rightarrow V \mid H \subset \operatorname{Ker}(f)\} .
\end{aligned}
$$

Lemma 43. Let $H \subset V$. Then $I_{V} \in H \otimes_{\mathbb{k}} V^{*}+V \otimes_{\mathbb{k}} H^{\perp}$.

Proof. Let $\mathfrak{p}: V \rightarrow H$ be any projector on $H$. Then $\operatorname{Id}_{V}=\mathfrak{p}+\left(\operatorname{Id}_{V}-\mathfrak{p}\right)$ and $\operatorname{Im}(\mathfrak{p})=$ $\operatorname{Ker}\left(\operatorname{Id}_{V}-\mathfrak{p}\right)=H$. Hence $\varphi^{-1}(\mathfrak{p}) \in H \otimes_{\mathbb{k}} V^{*}$ and $\varphi^{-1}\left(\operatorname{Id}_{V}-\mathfrak{p}\right) \in V \otimes_{\mathbb{k}} H^{\perp}$.

A semi-simple analogue. Let $\mathbf{C}_{0}$ be as in Subsection 2.3. and let $V_{\mathbf{C}_{0}}$ be an arbitrary right $\mathbf{C}_{0}$-module. Define

$$
\mathbf{C}_{0} V^{*}=\mathbf{C}_{0}-\operatorname{Mod}\left(V_{\mathbf{C}_{0}},\left(\mathbf{C}_{0}\right)_{\mathbf{C}_{0}}\right)
$$

(note that the authors of [BGS, 2.7] use the notation ${ }^{*} V$ for the same object). The formula (A.1) defines a canonical isomorphism,

$$
\varphi: V_{\mathbf{C}_{0}} \otimes \mathbf{C}_{0} \mathbf{C}_{0} V^{*} \stackrel{\sim}{\rightarrow} \operatorname{Hom}_{\mathbf{C}_{0}}\left(V_{\mathbf{C}_{0}}, V_{\mathbf{C}_{0}}\right) .
$$

Let $H_{\mathbf{C}_{0}} \subset V_{\mathbf{C}_{0}}$ be a (right) submodule. Then $\mathbf{C}_{0} H^{\perp}$ is a (left) submodule of $\mathbf{C}_{0} V^{*}$, and, analogously to Lemma 43, we obtain

$$
I_{V}:=\varphi^{-1}\left(\operatorname{Id}_{V}\right) \in H_{\mathbf{C}_{0}} \otimes \mathbf{C}_{0} \mathbf{C}_{0} V^{*}+V_{\mathbf{C}_{0}} \otimes \mathbf{C}_{0} \mathbf{C}_{0} H^{\perp} .
$$

A differential vector space for quadratic duals. Now let $\mathbf{C}$ and $\mathbf{C}^{!}$be as in Section 4. Let $M_{\mathbf{C}}$ be a right $\mathbf{C}$-module and ${ }_{\mathbf{C}^{!}} N$ be a left $\mathbf{C}^{!}$-module. Let $\left\{a_{i}: i=1, \ldots, k\right\}$ be a basis of $\mathbf{C}_{1}$ and $\left\{a^{i}: i=1, \ldots, k\right\}$ the corresponding dual basis of $\mathbf{C}_{1}^{!}$.

Proposition 44. The linear transformation

$$
\begin{aligned}
\delta: \quad M_{\mathbf{C}} \otimes_{\mathbf{C}_{0} \mathbf{C}^{!} N} & \rightarrow M_{\mathbf{C}} \otimes_{\mathbf{C}_{0} \mathbf{C}^{!}} N, \\
m \otimes n & \mapsto \sum_{i=1}^{k} m a_{i} \otimes a^{i} n
\end{aligned}
$$

satisfies $\delta^{2}=0$. Moreover, if both, $M_{\mathbf{C}}$ and $\mathbf{C}^{!} N$, are graded modules, then $M_{\mathbf{C}} \otimes_{\mathbf{C}_{0}}$ $\mathbf{C}^{!} N$ has a canonical bigrading, and $\delta$ is a homogeneous map of bidegree $(1,1)$.

Proof. That $\delta$ is a homogeneous map of bidegree $(1,1)$ in the graded situation is clear from the definition. What we have to prove is that $\delta^{2}=0$. Let $\mathbf{m}$ denote the multiplication in $\mathbf{C}$ (see Subsection 4.1), and $\mathbf{m} !$ denote the multiplication in $\mathbf{C}^{!}$. We have

$$
\begin{aligned}
\delta^{2}(m \otimes n) & =\sum_{i=1}^{k} \sum_{j=1}^{k} m a_{i} a_{j} \otimes a^{j} a^{i} n \\
& =m\left(\left(\mathbf{m} \otimes \mathbf{m}^{!}\right)\left(\sum_{i=1}^{k} \sum_{j=1}^{k}\left(a_{i} \otimes a_{j}\right) \otimes\left(a^{j} \otimes a^{i}\right)\right)\right) n \\
& =m\left(\left(\mathbf{m} \otimes \mathbf{m}^{!}\right) I_{\mathbf{C}_{1} \otimes \mathbf{C}_{0}} \mathbf{C}_{1}\right) n .
\end{aligned}
$$


Now let $R \subset \mathbf{C}_{1} \otimes_{\mathbf{C}_{0}} \mathbf{C}_{1}$ be the set of quadratic relations of $\mathbf{C}$. Then $R^{\perp}$ is the set of defining quadratic relations of $\mathbf{C}^{!}$by definition. Then, by (A.2) we have $I_{\mathbf{C}_{1} \otimes \mathbf{C}_{0}} \mathbf{C}_{1}=X+Y$, where

$$
X \in R \otimes \mathbf{C}_{0} \mathbf{C}_{1}^{*} \otimes \mathbf{C}_{0} \mathbf{C}_{1}^{*}, \quad Y \in \mathbf{C}_{1} \otimes \mathbf{C}_{0} \mathbf{C}_{1} \otimes \mathbf{C}_{0} R^{\perp} .
$$

Hence $\mathbf{m} \otimes \mathbf{m}^{!}(X)=0$ and $\mathbf{m} \otimes \mathbf{m}^{!}(Y)=0$ and thus $\delta^{2}(m \otimes n)=0$. This completes the proof.

Consider the vector space $\mathcal{C}^{\bullet}=\mathcal{C}^{\bullet}(M, N)$ defined via $\mathcal{C}^{i}=\left(M_{\mathbf{C}} \otimes_{\mathbf{C}_{0}} \mathbf{C}^{!} N\right)$ for all $i \in \mathbb{Z}$ (which means that we just place a copy of $M_{\mathbf{C}} \otimes_{\mathbf{C}_{0}} \mathbf{C}^{!} N$ in each position).

Corollary 45. (i) The linear transformation

$$
\begin{array}{lllc}
\delta: & \mathcal{C}^{\bullet} & \rightarrow & \mathcal{C}^{\bullet} \\
& \mathcal{C}^{i} \ni(m \otimes n) & \mapsto & \left(\sum_{i=1}^{k} m a_{i} \otimes a^{i} n\right) \in \mathcal{C}^{i+1}
\end{array}
$$

satisfies $\delta^{2}=0$, in particular, $\mathcal{C}^{\bullet}$ is a complex.

(ii) If $V$ is a $\mathbf{C}$-bimodule and $W$ is a $\mathbf{C}^{!}$-bimodule, then $\mathcal{C} \bullet(V, W)$ is a complex of C-C'-bimodules.

Finally, assume that both $\mathrm{M}$ and $\mathrm{N}$ are graded modules. Set $\mathcal{C}^{i}=\mathrm{M}_{\mathbf{C}}\langle i\rangle \otimes_{\mathbf{C}_{0}}$ $\mathrm{C}^{!} \mathrm{N}\langle i\rangle$ for all $i \in \mathbb{Z}$.

Corollary 46. (i) The linear transformation from Corollary 45)(ii) defines on $\mathcal{C}^{\bullet}$ the structure of a complex of graded vector spaces (i.e. the differential is a homogeneous map of degree 0 ).

(ii) If $\mathrm{V}$ is a graded $\mathbf{C}$-bimodule and $\mathrm{W}$ is a graded $\mathbf{C}^{!}$-bimodule, then $\mathcal{C} \bullet(\mathrm{V}, \mathrm{W})$ is a complex of bigraded $\mathbf{C}-\mathbf{C}^{!}$-bimodules.

Generalized Koszul complexes. Several known complexes can be obtained by this technique, for example:

- The complex $\mathcal{C}^{\bullet}\left(\mathbf{C}, \mathbf{C}^{\prime}\right)$, given by Corollary 46(1ii), is isomorphic to $\mathbb{P}^{\bullet}$ from Subsection 4.2 by construction.

- The complex $\mathcal{C}^{\bullet}\left(\mathbf{C},\left(\mathbf{C}^{!}\right)^{*}\right)$, given by Corollary 46)(iii), contains the classical Koszul complex (as in [BGS, 2.8]) as a subcomplex (of C-modules). In particular, $\mathcal{C}^{\bullet}\left(\mathbf{C},\left(\mathbf{C}^{!}\right)^{*}\right)$ can be considered as a natural bimodule extension of the Koszul complex.

Because of the last example it is natural to call the complexes, given by Corollary 46(ii), generalized Koszul complexes.

\section{ACKNOWLEDGMENTS}

We would like to thank Joseph Chuang for posing a question (at a conference in May 2005) which became the starting point for the present paper. We are deeply grateful to Bernhard Keller for many helpful suggestions and discussions. Special thanks to the referee for all her/his work and suggestions on improving the paper, and for fruitful additional mathematical discussions which we highly appreciate.

\section{REFERENCES}

[AS] H. H. Andersen, C. Stroppel, Twisting functors on $\mathcal{O}$, Represent. Theory 7 (2003), 681-699 (electronic). MR2032059 (2004k:17010) 
[Au] M. Auslander, Representation theory of Artin algebras. I, II. Comm. Algebra 1 (1974), 177-268; 1 (1974), 269-310. MR0349747 (50:2240)

[AR] M. Auslander, I.Reiten, Stable equivalence of dualizing R-varieties. Advances in Math. 12 (1974), 306-366. MR0342505 (49:7251)

[Ba1] E. Backelin, Koszul duality for parabolic and singular category $\mathcal{O}$, Represent. Theory 3 (1999), 139-152 (electronic). MR1703324(2001c:17034)

[Ba2] E. Backelin, The Hom-spaces between projective functors, Represent. Theory 5 (2001), 267-283 (electronic). MR1857082 (2002f:17007)

[Bass] H. Bass, Algebraic K-theory, Benjamin, New York-Amsterdam, 1968. MR0249491 $(40: 2736)$

[BGS] A. Beilinson, V. Ginzburg, W. Soergel, Koszul duality patterns in representation theory, J. Amer. Math. Soc. 9 (1996), no. 2, 473-527. MR1322847 (96k:17010)

[BFK] J. Bernstein, I. Frenkel, M. Khovanov, A categorification of the Temperley-Lieb algebra and Schur quotients of $U\left(\mathfrak{s l}_{2}\right)$ via projective and Zuckerman functors, Selecta Math. (N.S.) 5 (1999), no. 2, 199-241. MR1714141 (2000i:17009)

[BG] J. Bernstein, S. Gelfand, Tensor products of finite- and infinite-dimensional representations of semisimple Lie algebras, Compositio Math. 41 (1980), no. 2, 245-285. MR.581584 (82c:17003)

[BGG1] I. Bernštern, I. Gelfand, S. Gelfand, Algebraic vector bundles on $\mathbf{P}^{n}$ and problems of linear algebra, Funktsional. Anal. i Prilozhen., 12, (1978), no. 3, 66-67. MR509387 (80c:14010a)

[BGG2] I. Bernštern, I. Gelfand, S. Gelfand, A certain category of $\mathfrak{g}$-modules, Funkcional. Anal. i Priložen. 10 (1976), no. 2, 1-8. MR0407097 (53:10880)

[BoGa] K. Bongartz, P. Gabriel, Covering spaces in representation theory. Invent. Math. 65 (1981/82), no. 3, 331-378. MR643558(84i:16030)

[Br] G. Bredon, Equivariant cohomology theories, Lecture Notes in Math, 34, 1967. MR0206946 (34:6762)

[CM] C. Cibils, E. Marcos, Skew category, Galois covering and smash product of a $k$-category, Proc. AMS, 134 (2006), no.1, 39-50. MR.2170541 (2006e:18003)

[De] P. Deligne, Cohomologie a support propre et construction du foncteur $f^{!}$, Lecture Notes in Mathematics 20, 1966, pp. 404-423.

[tDi] T. tom Dieck, Über projektive Moduln und Endlichkeitshindernisse bei Transformationsgruppen, Manuscripta Mathematica 34 (1981), 135-155. MR620445 (82k:57028)

[Fi1] P. Fiebig, Centers and translation functors for the category $\mathcal{O}$ over Kac-Moody algebras, Math. Z. 243 (2003), no. 4, 689-717. MR1974579 (2004c:17051)

[Fi2] P. Fiebig, The combinatorics of category $\mathcal{O}$ for symmetrizable Kac-Moody algebras, Transf. Groups. 11 (2006), no. 1, 29-49. MR.2205072 (2006k:17040)

[Fl] G. Fløystad, Koszul duality and equivalences of categories, Trans. Amer. Math. Soc. 358 (2006), no. 6, 2373-2398. MR2204036 (2006m:16028)

[FKS] I. Frenkel, M. Khovanov, C. Stroppel, A categorification of finite-dimensional irreducible representations of quantum sl(2) and their tensor products, Slecta Mathematica (N.S.) 12 (2006), no. 3-4, 379-431. MR.2305608 (2008a:17014)

[GJ] O. Gabber, A. Joseph, Towards the Kazhdan-Lusztig conjecture, Ann. Sci. École Norm. Sup. (4) 14 (1981), no. 3, 261-302. MR644519 (83e:17009)

[Ga] P. Gabriel, Des catégories abéliennes, Bull. Soc. Math. France 90 (1962), 323-448. MR.0232821 (38:1144)

[GM] S. Gelfand, Y. Manin, Methods of homological algebra, Second edition, Springer Monographs in Mathematics. Springer-Verlag, Berlin, 2003. MR.1950475 (2003m:18001)

[GK] V. Ginzburg, M. Kapranov, Koszul duality for operads, Duke Math. J. 76 (1994), no. 1, 203-272. MR1301191 (96a:18004)

[GMRSZ] E. Green, R. Martínez-Villa, I. Reiten, Ø. Solberg, D. Zacharia, On modules with linear presentations, J. Algebra 205 (1998), no. 2, 578-604. MR1632765 (99i:16075)

[GRS] E. Green, I. Reiten, Ø. Solberg, Dualities on generalized Koszul algebras, Mem. Amer. Math. Soc. 159 (2002), no. 754. MR1921583(2004b:16042)

[Ha] D. Happel, Triangulated categories in the representation theory of finite-dimensional algebras, London Mathematical Society Lecture Note Series, 119, Cambridge University Press, Cambridge, 1988. MR935124 (89e:16035) 
[HI] J. Herzog, S. Iyengar, Koszul modules. J. Pure Appl. Algebra 201 (2005), no. 1-3, 154-188. MR 2158753 (2006d:13013)

[Ir] R. Irving, Shuffled Verma modules and principal series modules over complex semisimple Lie algebras, J. London Math. Soc. (2) 48 (1993), no. 2, 263-277. MR:1231714 (94i:17013)

[Ja1] J. C. Jantzen, Moduln mit einem höchsten Gewicht, Lecture Notes in Mathematics, 750, Springer, Berlin, 1979. MR552943 (81m:17011)

[Ja2] J. C. Jantzen, Einhüllende Algebren halbeinfacher Lie-Algebren, Ergebnisse der Mathematik und ihrer Grenzgebiete (3), 3, Springer-Verlag, Berlin, 1983. MR721170 (86c:17011)

[Jo] A. Joseph, The Enright functor on the Bernstein-Gelfand-Gelfand category $\mathcal{O}$, Invent. Math. 67 (1982), no. 3, 423-445. MR664114 (84j:17005)

[KS] M. Kashiwara, P. Schapira, Sheaves on manifolds, Grundlehren der Mathematischen Wissenschaften, 292, Springer-Verlag, Berlin, 1994. MR1299726 (95g:58222)

[Ke1] B. Keller, On the construction of triangle equivalences, in: Derived equivalences for group rings. Lecture Notes in Math., 1685, 155-176, 1998. MR.1649844

[Ke2] B. Keller, Deriving DG categories, Ann. Sci. École Norm. Sup. (4) 27 (1994), no. 1, 63-102. MR.1258406 (95e:18010)

[Ke3] B. Keller, Koszul duality and coderived categories (after K. Lefèvre), Preprint 2003.

[KM] O. Khomenko, V. Mazorchuk, On Arkhipov's and Enright's functors, Math. Z. 249 (2005), no. 2, 357-386. MR2115448 (2005k:17004)

$[\mathrm{McL}] \quad$ S. Mac Lane, Homology. Reprint of the 1975 edition. Classics in Mathematics. SpringerVerlag, Berlin, 1995. MR1344215 (96d:18001)

[MVS] R. Martínez Villa, M. Saorín, Koszul equivalences and dualities. Pacific J. Math. 214 (2004), no. 2, 359-378. MR2042938 (2005e:16046)

[MVZ] R. Martínez Villa, D. Zacharia, Approximations with modules having linear resolutions. J. Algebra 266 (2003), no. 2, 671-697. MR1995131 (2004h:16005)

[Ma] V. Mazorchuk, Applications of the category of linear complexes of tilting modules associated with the category $\mathcal{O}$, math.RT/0501220, to appear in Alg. Rep. Theory.

[MO] V. Mazorchuk and S. Ovsienko, A pairing in homology and the category of linear complexes of tilting modules for a quasi-hereditary algebra, J. Math. Kyoto Univ. 45 (2005), no. 4, 711-741. MR2226627 (2007d:16015)

[MS1] V. Mazorchuk and C. Stroppel, On functors associated to a simple root, J. Algebra 314 (2007), no. 1, 97-128. MR2331754

[MS2] V. Mazorchuk and C. Stroppel, Translation and shuffling of projectively presentable modules and a categorification of a parabolic Hecke module, Trans. Amer. Math. Soc. 357 (2005), no. 7, 2939-2973. MR.2139933 (2006g:17012)

[MS3] V. Mazorchuk and C. Stroppel, A combinatorial approach to functorial quantum sl( $k$ ) knot invariants, arXiv:0709.1971.

[Mi] B. Mitchell, Rings with several objects. Advances in Math. 8 (1972), 1-161. MR0294454 $(45: 3524)$

[Ri] J. Rickard, Morita theory for derived categories. J. London Math. Soc. (2) 39 (1989), no. 3, 436-456. MR1002456 (91b:18012)

[RH] S. Ryom-Hansen, Koszul duality of translation and Zuckerman functors. J. Lie Theory 14 (2004), no. 1, 151-163. MR2040174 (2005g:17018)

[Sc] H. Schubert, Kategorien. I, Heidelberger Taschenbücher, 66, Springer, Berlin, 1970. MR 0274548 (43:311)

[Sh] U. Shukla, On the projective cover of a module and related results, Pacific J. Math. 12 1962 709-717. MR0146235 (26:3757)

[So] W. Soergel, Kategorie $\mathcal{O}$, perverse Garben und Moduln über den Koinvarianten zur Weylgruppe, J. Amer. Math. Soc. 3 (1990), no. 2, 421-445. MR1029692 (91e:17007)

[St1] C. Stroppel, Category $\mathcal{O}$ : gradings and translation functors, J. Algebra 268 (2003), no. 1, 301-326. MR2005290 (2004i:17007)

[St2] C. Stroppel, Categorification of the Temperley-Lieb category, tangles, and cobordisms via projective functors, Duke Math. J. 126 (2005), no. 3, 547-596. MR2120117 (2005i:17011) 
[St3] C. Stroppel, TQFT with corners and tilting functors in the Kac-Moody case, arXive:math/0605103.

[Su] J. Sussan, Category $\mathcal{O}$ and $\operatorname{sl}(k)$ link invariants, arXive:math/0701045.

Department of Mathematics, Uppsala University, Box 480, 751 06, Uppsala, Sweden

E-mail address: mazor@math.uu.se

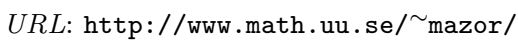

Department of Mathematics, Kyiv University, 64, Volodymyrska st., 01033, Kyiv, UKRAINE

E-mail address: ovsko@voliacable.net

Department of Mathematics, University of Glasgow, University Gardens, Glasgow G12 8QW, UNITED Kingdom

E-mail address: cs@maths.gla.ac.uk

$U R L:$ http://www.maths.gla.ac.uk/ cs/ 